\title{
Optimal Investment Strategies for Flexible Resources, Considering Pricing and Correlated Demands
}

\author{
Qiong Wang \\ Grado Department of Industrial and Systems Engineering \\ Virginia Polytechnic Institute and State University \\ Thesis Submitted to the Faculty of \\ Virginia Polytechnic Institute and State University \\ in fulfillment of the requirements for the degree of \\ Master of Science \\ In \\ Industrial and Systems Engineering \\ Ebru K. Bish, Chair \\ Philip Y. Huang \\ Charles P. Koelling
}

November 2002

Blacksburg, Virginia

Keywords: Resource investment decision under uncertainty, resource flexibility, pricing, stochastic 


\title{
Optimal Investment Strategies for Flexible Resources, Considering Pricing and Correlated Demands
}

\author{
Qiong Wang
}

\begin{abstract}
We study the resource investment decision faced by a firm that offers two demandclasses (i.e., products, services), while incorporating the firm's pricing decision into the investment decision. For this purpose, we consider a monopolistic situation and model the demand curve of each demand-class as a downward sloping linear function of its own price. The firm can invest in dedicated resources, which can only satisfy a specific demand-class, and/or in a more expensive, flexible resource, which can satisfy both demand-classes.

We consider a two-stage stochastic decision model: In the first stage, the firm determines the dedicated and flexible resource capacities to invest in under demand uncertainty. In the second stage, demand curves are realized and the firm optimizes its revenue through pricing and resource allocation decisions, constrained by its capacity investment decision in the first stage.

Our analysis provides the structure of the firm's optimal resource investment strategy as a function of price elasticities and investment costs, and shows how the value of resource flexibility depends on these parameters and demand correlations. Based on our analysis, we provide principles on the firm's optimal resource investment strategy under uncertainty.

We show that it can be optimal for the firm to invest in the flexible resource when demand patterns are perfectly positively correlated, while it is not always optimal to invest in the flexible resource when demand patterns are perfectly negatively correlated.
\end{abstract}




\section{Acknowledgments}

I am deeply indebted to my advisor, Dr. Bish. Without her guidance, support, and good nature, I would never have been able to complete this thesis. I thank Dr. Bish for her insight, depth of knowledge, dedication, and patience as an advisor; and her encouragement, trust, and respect as a friend throughout my graduate program. I learned a lot of knowledge and life from her. I also thank my committee members, Dr. Huang and Dr. Koelling, for their valuable help and stimulating discussions, comments on my thesis despite their extremely busy schedule.

I sincerely thank the faculty and staff in the Grado Department of Industrial and Systems Engineering with a special mention to Ms. Lovedia Cole for providing me an excellent work environment that has nurtured me as a good graduate student.

I also want to express my appreciation to Alexander Walter Scholarship, teaching and research assistance that made my dream of studying in VT came true.

I am grateful to all my friends who helped me and gave me a very pleasant and meaningful time during my two years' life in Blacksburg.

Finally, a special note of love and gratitude goes to my husband, Chendong. All his love, encouragement and consideration were strong motivations for me to pursue my MS. Also, I would like to thank my mother. The only time I can share with her in this two years was a few phone calls. I still recall clearly her tearful eyes when I left China. 


\section{Contents}

Acknowledgments

1 Introduction 1

1.1 Introduction and Motivation . . . . . . . . . . . . 1

1.2 Research Objectives . . . . . . . . . . . . . . . . . 2

1.3 Research Approach . . . . . . . . . . . . . . . . . . 3

2 Literature Review 4

3 Model, Notation, and Preliminaries $\quad 8$

4 Characterization of the Optimal Pricing and Resource Allocation Strategy in Stage 2

5 Characterization of the Optimal Investment Strategy in Stage $1 \quad 19$

6 Impact of Demand Correlation on the Optimal Investment Strategy 28

6.1 Perfectly Positively Correlated Demand Patterns . . . . . . . . . . . . . 29

6.2 Perfectly Negatively Correlated Demand Patterns . . . . . . . . . . . . . . 34

7 Conclusions and Future Research Directions 36

$\begin{array}{ll}\text { References } & 40\end{array}$

$\begin{array}{ll}\text { Appendix } & 42\end{array}$

A Proof of Lemma $4.1 \ldots \ldots \ldots \ldots \ldots$ 
B Proof of Lemma $5.1 \ldots \ldots \ldots \ldots \ldots$

C Proof of Lemma $5.3 \ldots \ldots \ldots \ldots \ldots$

D Proof of Lemma $6.2 \ldots \ldots \ldots \ldots \ldots \ldots$

D.1 Case 1: $\frac{\alpha_{1} c_{1}}{a}>\alpha_{2} c_{2} \ldots \ldots \ldots \ldots \ldots \ldots$

D.2 Case 2: $\frac{\alpha_{1} c_{1}}{a}=\alpha_{2} c_{2} \ldots \ldots \ldots \ldots \ldots \ldots$

D.3 Case $3: \frac{\alpha_{1} c_{1}}{a}<\alpha_{2} c_{2} \ldots \ldots \ldots \ldots \ldots \ldots$

E Proof of Theorem 6.1 parts $(2 \mathrm{a})$ and $(2 \mathrm{~b}) \ldots \ldots \ldots \ldots$

E.1 Proof of $(2 \mathrm{a}) \ldots \ldots \ldots \ldots \ldots \ldots \ldots$

E.2 Proof of $(2 \mathrm{~b}) \ldots \ldots \ldots \ldots \ldots \ldots$

F Proof of Theorem $6.2 \ldots \ldots \ldots \ldots \ldots$

$\begin{array}{lr}\text { Vita } & 80\end{array}$ 


\section{List of Figures}

1 The demand space for $\left(\epsilon_{1}, \epsilon_{2}\right)$ in Stage $2 \ldots \ldots \ldots \ldots$

2 The demand space at solution $\vec{K}^{2 F}$ for perfectly positively correlated demand patterns. .......................... 64

3 The demand space at solution $\vec{K}^{A}$ for perfectly positively correlated demand patterns. . . . . . . . . . . . . . . . . . 70

4 The demand space at solution $\vec{K}^{A}$ for case $1 . \ldots \ldots \ldots$. . . . . . . 74

5 The demand space at solution $\vec{K}^{A}$ for case $2 \ldots \ldots \ldots \ldots$ 


\section{Chapter 1}

\section{Introduction}

\subsection{Introduction and Motivation}

The resource investment decision in several industries is characterized by long investment lead times (for example, 3-5 years in the automotive industry) and economies of scale in investment costs. These necessitate the resource investment decision to be made early, under high demand uncertainty. An example is the automotive industry, where the average deviation of the demand forecast in the capacity investment stage from the actual sales is about 40\% [Bish et al. (2001), Jordan and Graves (1995)].

One strategy that can be used to hedge against demand uncertainty in the investment stage is to invest in "flexible resources," where flexible resources refer to the resources with the ability to satisfy multiple "demand-classes" (i.e., products, services). Investments in resource flexibility enable the firm to balance its supply with demand more effectively, while reducing inter-period inventories, and enhance its competitiveness in the market place.

Since flexible resources are more expensive to invest in than dedicated resources, effective strategies for designing and managing flexible resources are highly valuable for 
management. Unfortunately, research that focuses on resource flexibility and analyzes its value is very limited. Today's market place is becoming more and more uncertain and extremely competitive. Therefore, there is a great need to understand the structure of the firm's optimal strategy for investing in flexible resources in order to get insights into managerial level decisions.

On the other hand, demand-side controls, such as pricing, can offer another valuable strategy to reduce supply-demand imbalances. Most previous research that has studied the value of resource flexibility has ignored the effect of pricing on the firm's resource investment decision. Therefore, in this research, we would like to answer the following research questions: When should we invest in flexible resources? What is the structure of the firm's optimal investment and pricing strategy? How does the firm's optimal investment strategy depend on demand correlations and price elasticities?

The next section presents our research objectives.

\subsection{Research Objectives}

Our research objectives are:

- To understand the structure of the firm's optimal resource investment strategy under demand uncertainty;

- To understand how the optimal investment strategy depends on price elasticities and demand correlations;

- To offer managerial guidelines and principles on the firm's optimal resource investment decision based on the insights obtained. 


\subsection{Research Approach}

We consider a two demand-class problem. In particular, we consider two demand-classes, since this case is analytically tractable, while being sufficient to capture the important elements of the problem. In this research, a two-stage stochastic decision model is developed, where in the first stage (a long time before production starts), the firm determines its resource investment under demand uncertainty. The shapes of the demand curves are known in stage 1, but the locations of the curves are unknown. In the second stage (a short time before production starts), the firm optimizes its profit through pricing and resource allocation decisions, which are constrained by its resource investment in the first stage. Our objective is to analyze the interactions between the firm's optimal investment strategy, pricing, and demand correlations so that we can provide principles and guidelines on the firm's optimal investment strategy at the managerial level.

This thesis is organized as follows. In Chapter 2, we present a brief review of the related literature. In Chapter 3, we introduce our model and assumptions. Chapters 4 and 5 provide our analysis on the characterization of the optimal pricing and resource allocation strategy in stage 2 , and the optimal investment strategy in stage 1 , respectively. In Chapter 6 , we discuss the impact of demand correlations, i.e., perfectly positively correlated and perfectly negatively correlated demand patterns, on the value of resource flexibility. Finally, Chapter 7 presents the summary of the contributions in this study and suggestions on future research directions. 


\section{Chapter 2}

\section{Literature Review}

There are two papers that are very related to this research: the papers by Fine and Freund (1990) and Van Mieghem (1998), in the sense that both papers address the resource investment problem for a two demand-class problem using a two-stage stochastic programming formulation. In what follows, we refer to Fine and Freund (1990) as FF and Van Mieghem (1998) as VM.

FF study the firm's optimal resource investment strategy in the presence of flexible resources. FF formulate the problem as a two-stage stochastic programming decision model. In the first stage, the firm makes its resource investment decision under demand uncertainty, which is characterized by a set of possible scenarios. In the second stage, the firm optimizes its profit, given realizations of demand. FF characterize the optimal resource investment decision for the two demand-class case. They also provide numerical analysis on the sensitivity of the firm's optimal capacity investment decision to the investment costs and demand correlations. In FF's study, price elasticity is implicitly considered through concave revenue functions. Although our model is similar to theirs, we consider demand 
uncertainty through a continuous error term rather than a set of possible scenarios. Based on this, we build a new characterization of the firm's optimal investment strategy and its sensitivity to various parameters such as price elasticities of demand, investment costs and demand correlations.

VM studies a similar problem, considering that the firm is a price-taker, i.e., prices are exogenously determined. VM also formulates the problem as a two-stage decision problem. In stage 1, the firm determines its optimal resource investment strategy before the resolution of the demand uncertainty. In stage 2 , i.e., when demands are realized, the firm decides on the allocation of the resources to the two demand-classes. VM uses a multidimensional newsvendor model to characterize the firm's optimal investment strategy. In that sense, our model can be considered an extension of VM's work to incorporate the pricing decision into the optimal investment strategy. VM states that the flexible resource can be considered as an optimal cost/benefit response to the variability in demand. $\mathrm{He}$ analyzes the perfectly positive and negative correlations between the two demand-classes and demonstrates that "the optimal levels of dedicated capacity increase in a concave manner as correlation increases, while the optimal level of flexible capacity decreases in a convex manner (page 1078 in Van Mieghem (1998))." In addition, VM shows that when the prices are different, it can be advantageous to invest in the flexible resource even when demands are perfectly positively correlated.

"Flexibility" mentioned above can be seen as "full flexibility," since the flexible resource can be used to satisfy any demand-class. Some researchers have analyzed the "partial flexibility" or "limited flexibility" case, where the flexible resources can be used to satisfy only a subset of demand-classes. For example, Bassok, Anupindi and Akella (1999) consider the 
problem of determining the optimal order quantities in a multi-product inventory system that allows "full downward substitution," i.e., demand of one product can be substituted by any product with a higher value. In their model, the order quantities are determined under demand uncertainty, while the allocation of products to demands is determined after demands are realized. They study properties of the optimal strategy and show that it can be highly beneficial to consider the substitution possibility at the ordering stage, especially in cases of high demand variability, low substitution cost, low profit margins, and product similarity.

Another extension is considered by Netessine, Dobson and Shumsky (2002). Netessine, Dobson and Shumsky study a single-period two-stage stochastic system under exogenously determined prices. They consider only one-level substitution, present an analytical solution for the optimal resource investment levels, and characterize the impact of demand correlation on the optimal investment decision. Netessine, Dobson and Shumsky show that for two types of demand-classes, as the demand correlation increases, there is a shift from flexible resource to dedicated resources.

We want to note here that most of the existing literature considers prices as given while addressing the value of resource flexibility. An exception is the work by Birge, Drogosz and Duenyas (1998), who study the single-period newsvendor problem of determining the optimal resource investment level and prices for two substitutable products. However, in their model, it is the customers who substitute among the different demand-classes, and not the firm. See also Petruzzi and Dada (1999) for a review of pricing models for the single product case. 
Other relevant research includes research that addresses the optimal resource investment strategy in a multi-period setting, such as Caulkins and Fine (1990), Eberly and Van Mieghem (1997), and Harrison and Van Mieghem (1999); and in a multi-product multi-plant setting such as Jordan and Graves (1995) and Li and Tirupati (1994). 


\section{Chapter 3}

\section{Model, Notation, and Preliminaries}

We consider a firm that offers multiple demand-classes (i.e., service levels/products). The firm needs to determine its resource investment decision under high demand uncertainty, and then makes its pricing and resource allocation decision as demands are realized. We model this decision problem as a two-stage stochastic programming problem, as commonly done in the previous literature. In the first stage, the firm determines its resource investment under uncertainty so as to maximize its expected profit. In the second stage, uncertainty is resolved and the firm jointly determines its pricing and resource allocation to maximize its revenue. Thus, the firm utilizes an integrated resource flexibility/price postponement strategy in the second stage.

Our model can be characterized as a multi-dimensional newsvendor model with pricing [see, for instance, Petruzzi and Dada (1999) and Porteus (1990) for the single newsvendor problem]. In particular, we consider two demand-classes, since this case is analytically tractable, while being sufficient to capture the important elements of the problem. Thus, the firm has the option to invest in two dedicated resources, each of which can satisfy 
only one demand-class, and/or in a more expensive, flexible resource that can satisfy both demand-classes. We model the demand for each demand-class $i\left(d_{i}\right)$, as a downward-sloping linear function of its own price (or contribution margin), denoted as $p_{i}$. That is, for $i=1,2$,

$$
d_{i}=\xi_{i}-\alpha_{i} p_{i}
$$

where $\alpha_{i}>0$ is the price-elasticity of demand and $\xi_{i}$ is the intercept. In the first stage of our stochastic program, we model each $\xi_{i}, i=1,2$, as a continuous random variable with positive support in $[0, \infty)$; this represents the uncertainty in the market size (long-term uncertainty). Let $f_{i}(\cdot), F_{i}(\cdot)$, and $\bar{F}_{i}(\cdot)$ respectively denote the probability density function (pdf), cumulative distribution function (CDF), and the tail distribution of $\xi_{i}, i=1,2$. Throughout the thesis, we do not make any distributional assumptions on $\xi_{1}$ and $\xi_{2}$. Therefore, all the following results hold for any continuous distribution of $\xi_{1}$ and $\xi_{2}$ defined over $[0, \infty)$.

The firm seeks a coordinated resource investment, pricing, and allocation strategy to maximize its expected profit. In stage 1 , the values of random variables $\vec{\xi}=\left(\xi_{1}, \xi_{2}\right)$ are uncertain. At this time, the firm makes its resource investment decision, $\vec{K}=\left(K_{1}, K_{2}, K_{f}\right)$, so as to maximize its expected profit, where $K_{i}$ corresponds to the investment level for dedicated resource $i, i=1,2$, and $K_{f}$ that for the flexible resource. Let $K_{T} \equiv K_{1}+K_{2}+$ $K_{f}$ and $V(\vec{K})$ denote the expected profit in stage 1, which equals the expected revenue $(E[\Pi(\vec{K}, \vec{\xi})])$ less the investment costs. Then, in stage 2 uncertainty is resolved (i.e., the realization $\epsilon_{i}$ of random variable $\xi_{i}$ is observed for $\left.i=1,2\right)$ and the firm maximizes its profit through pricing $\left(p_{i}, i=1,2\right)$ and resource allocation decisions, constrained by its 
earlier investment decision. Let $\vec{x}=\left(y_{1}, y_{2}, z_{1}, z_{2}\right)$ denote the resource allocation vector in stage 2 , where $y_{i}$ and $z_{i}$ respectively correspond to the amount of class $i$ demand satisfied using the dedicated resource and the flexible resource, for $i=1,2$. As in the earlier literature, we assume that investment costs are linear - all our results readily extend to convex investment costs - and that the variable cost of satisfying a demand-class is the same for the dedicated and the flexible resource. Let $c_{i}$ denote the unit cost of investing in resource $K_{i}, i=1,2, f$, where $c_{1}, c_{2}<c_{f}$. In addition, we consider that $c_{f}<c_{1}+c_{2}$; otherwise the problem becomes trivial (i.e., we never invest in the flexible resource).

This decision problem can be formulated as the following stochastic program:

$$
\begin{array}{lll}
\text { (Stage 1 Problem) } P_{1}: & \max _{\vec{K} \geq 0} \quad V(\vec{K})=E[\Pi(\vec{K}, \vec{\xi})]-\sum_{i=1,2, f} c_{i} K_{i} \\
\text { (Stage 2 Problem) } P_{2}: \quad & \max _{\vec{x}, \vec{p}} \Pi(\vec{K}, \vec{\epsilon})=\sum_{i=1}^{2} p_{i}\left(y_{i}+z_{i}\right) \\
& \text { subject to } \\
& y_{i} \leq K_{i}, \quad i=1,2 \\
& z_{1}+z_{2} \leq K_{f} \\
& y_{i}+z_{i} \leq \epsilon_{i}-\alpha_{i} p_{i}, \quad i=1,2 \\
& p_{i} \leq \frac{\epsilon_{i}}{\alpha_{i}}, \quad i=1,2 \\
& y_{i}, z_{i}, p_{i} \geq 0, \quad i=1,2
\end{array}
$$

In the above formulation, constraints (2) and (3) are the capacity constraints for the dedicated and flexible resources, respectively, whereas constraints (4) ensure that the total amount of each demand-class satisfied does not exceed its demand, induced by the firm's 
pricing decision. Finally, constraints (5) and (6) are the non-negativity constraints for demands, allocation quantities, and prices, respectively.

Observe that we can write constraints (4) as equalities, since any solution with excess demand would be sub-optimal for Problem $P_{2}$ (i.e., price can be further increased to reduce demand to the overall capacity level, resulting in a higher revenue), but of course, we can have excess capacity. Based on this observation, we can write the following equivalent formulation.

$$
\begin{array}{ll}
\text { (Stage 2 Problem) } P_{2}^{\prime}: \quad & \max _{\vec{p}} \Pi(\vec{K}, \vec{\epsilon})=\sum_{i=1}^{2} p_{i}\left(\epsilon_{i}-\alpha_{i} p_{i}\right) \\
& \text { subject to } \\
& p_{i} \geq \frac{\epsilon_{i}-K_{i}-K_{f}}{\alpha_{i}}, \quad i=1,2 \\
& \alpha_{1} p_{1}+\alpha_{2} p_{2} \geq \epsilon_{1}+\epsilon_{2}-K_{1}-K_{2}-K_{f} \longleftarrow \lambda_{i}
\end{array}
$$

where $\lambda_{i}, i=1,2$, and $\mu$ represent the corresponding Lagrangian multiplier for each constraint. Observe that we have omitted the demand non-negativity constraints, $y_{i}+z_{i}=$ $\epsilon_{i}-\alpha_{i} p_{i} \geq 0, i=1,2$, and price non-negativity constraints. The optimal solution will always satisfy them, since the amount of demand-class $i$ satisfied will be at least $\min \left(\frac{\epsilon_{i}}{2}, K_{i}\right) \geq 0$, which is the optimal solution in the absence of the flexible resource, as detailed below. Equivalently, the optimal price of demand-class $i$ will be at $\operatorname{most} \max \left(\frac{\epsilon_{i}}{2 \alpha_{i}}, \frac{\epsilon_{i}-K_{i}}{\alpha_{i}}\right)$. Thus, there exists a feasible solution, which generates a non-negative revenue for demand-class $i$ without affecting the available capacity for demand-class $j \neq i$, given by $K_{j}+K_{f}$. Hence, demand and price non-negativity constraints can be eliminated.

For convenience, we are going to use this equivalent formulation when we characterize 
the optimal solution to the stage 2 problem in the next section. In our formulation, we assume zero penalty cost for a lost sale rather than a forfeited profit. This is because the firm is a price-setter and determines how much demand to satisfy through pricing. However, a penalty cost per unsatisfied unit of demand or a salvage value for unused capacity can be included in the model without changing the structure of the results.

Finally, observe that when the flexible resource is not available or it is not considered in the investment decision, the investment decision will decompose by each demand-class, in which case the optimal dedicated resource capacity for each demand-class can be obtained independently. We will refer to this case as the "dedicated system." Below we introduce the formulation for each demand-class in the dedicated system and use it subsequently in our analysis.

$$
\begin{array}{lll}
\text { (Class } i \text { Stage } 1 \text { Problem) } P_{1}(i): & \max _{K_{i} \geq 0} V_{i}\left(K_{i}\right) \equiv E\left[\Pi_{i}\left(K_{i}, \xi_{i}\right)\right]-c_{i} K_{i} \\
\left(\text { Class } i \text { Stage } 2 \text { Problem) } P_{2}^{\prime}(i):\right. & \max _{p_{i}} \Pi_{i}\left(K_{i}, \epsilon_{i}\right)=p_{i}\left(\epsilon_{i}-\alpha_{i} p_{i}\right) \\
& \text { subject to } \\
& p_{i} \geq \frac{\epsilon_{i}-K_{i}}{\alpha_{i}}
\end{array}
$$

where $\gamma_{i}$ denotes the corresponding Lagrangian multiplier.

Let $p_{i}^{I}$ and $\gamma_{i}^{I}$ denote the optimal solution to demand-class $i^{\prime} s$ Stage 2 Problem, $P_{2}^{\prime}(i), i=1,2$. Since $\Pi_{i}$ is strictly concave in $p_{i}$, the first-order Karush-Kuhn-Tucker (KKT) conditions are necessary and sufficient for optimality, which lead to the following 
results.

$$
\begin{aligned}
& p_{i}^{I}=\max \left(\frac{\epsilon_{i}}{2 \alpha_{i}}, \frac{\epsilon_{i}-K_{i}}{\alpha_{i}}\right) \\
& \gamma_{i}^{I}=\frac{1}{\alpha_{i}}\left(2 \alpha_{i} p_{i}^{I}-\epsilon_{i}\right)^{+}= \begin{cases}\frac{1}{\alpha_{i}}\left(\epsilon_{i}-2 K_{i}\right), & \text { if } \epsilon_{i}>2 K_{i}, \\
0, & \text { otherwise. }\end{cases}
\end{aligned}
$$

We let $\vec{K}^{I}=\left(K_{1}^{I}, K_{2}^{I}\right)$ denote the optimal investment vector in the dedicated system. The following result will be used later in our analysis.

Lemma 3.1 Consider the optimal investment solution, $\vec{K}^{I}$, in the dedicated system. If $c_{i}<\frac{E\left[\xi_{i}\right]}{\alpha_{i}}, i=1,2$, then $K_{i}^{I}$, is the unique solution to:

$$
E\left[\xi_{i}-2 K_{i}^{I} \mid \xi_{i}>2 K_{i}^{I}\right] \operatorname{Pr}\left(\xi_{i}>2 K_{i}^{I}\right)=\alpha_{i} c_{i}
$$

Otherwise (if $\left.c_{i} \geq \frac{E\left[\xi_{i}\right]}{\alpha_{i}}\right), K_{i}^{I}=0$.

Proof: Using Eq. (11), the expected profit of demand-class $i, i=1,2$, in Stage 1 can be written as:

$$
\begin{aligned}
V_{i}\left(K_{i}\right) & =E\left[\Pi_{i}\left(K_{i}, \xi_{i}\right)\right]-c_{i} K_{i} \\
& =\int_{0}^{2 K_{i}} \frac{\epsilon_{i}^{2}}{4 \alpha_{i}} f_{i}\left(\epsilon_{i}\right) d \epsilon_{i}+\int_{2 K_{i}}^{\infty} \frac{K_{i}\left(\epsilon_{i}-K_{i}\right)}{\alpha_{i}} f_{i}\left(\epsilon_{i}\right) d \epsilon_{i}-c_{i} K_{i}
\end{aligned}
$$

By Leibniz's rule,

$$
\begin{aligned}
\frac{\delta V_{i}\left(K_{i}\right)}{\delta K_{i}} & =\frac{\delta E\left[\Pi_{i}\left(K_{i}, \xi_{i}\right)\right]}{\delta K_{i}}-c_{i}=\int_{2 K_{i}}^{\infty} \frac{\left(\epsilon_{i}-2 K_{i}\right)}{\alpha_{i}} f_{i}\left(\epsilon_{i}\right) d \epsilon_{i}-c_{i} \\
\Rightarrow & \frac{\delta^{2} V_{i}\left(K_{i}\right)}{\delta K_{i}^{2}}=\frac{-2}{\alpha_{i}} \bar{F}_{i}\left(2 K_{i}\right)<0, \text { for any } K_{i}>0
\end{aligned}
$$

Thus, $V_{i}\left(K_{i}\right)$ is strictly concave in $K_{i}$, for $K_{i}>0$. Observe that if $\left.\frac{\delta E\left[\Pi_{i}\left(K_{i}, \xi_{i}\right)\right]}{\delta K_{i}}\right|_{K_{i}=0} \leq c_{i}$, 
or equivalently, if $c_{i} \geq \frac{E\left[\xi_{i}\right]}{\alpha_{i}}$, then it is not economically viable to invest in any dedicated capacity for demand-class $i$ in Stage 1 , and $K_{i}^{I}=0$. Otherwise, $K_{i}^{I}$ is the unique solution to:

$$
\begin{aligned}
& \frac{\delta V_{i}\left(K_{i}\right)}{\delta K_{i}}=\int_{2 K_{i}}^{\infty} \frac{\left(\epsilon_{i}-2 K_{i}\right)}{\alpha_{i}} f_{i}\left(\epsilon_{i}\right) d \epsilon_{i}-c_{i}=0 \\
\Rightarrow & E\left[\xi_{i}-2 K_{i}^{I} \mid \xi_{i}>2 K_{i}^{I}\right] \operatorname{Pr}\left(\xi_{i}>2 K_{i}^{I}\right)=\alpha_{i} c_{i},
\end{aligned}
$$

which completes the proof. 


\section{Chapter 4}

\section{Characterization of the Optimal Pricing}

\section{and Resource Allocation Strategy in}

\section{Stage 2}

In the following, we first characterize the optimal solution to Problem $P_{2}^{\prime}$, the joint Stage 2 Problem for the two demand-classes, introduced in the previous section. For this purpose, given a resource capacity vector, $\vec{K}$, we decompose the demand space into the following disjoint sets; see Figure 1.

$$
\begin{aligned}
& \Omega_{1}=\left\{\xi_{1}<2 K_{1}+2 K_{f}, \quad \xi_{2}<2 K_{2}+2 K_{f}, \quad \xi_{1}+\xi_{2}<2 K_{1}+2 K_{2}+2 K_{f}\right\} \\
& \Omega_{2}=\left\{2 \alpha_{1} K_{2}-2 \alpha_{2}\left(K_{1}+K_{f}\right)<\alpha_{1} \xi_{2}-\alpha_{2} \xi_{1}<-2 \alpha_{2} K_{1}+2 \alpha_{1}\left(K_{2}+K_{f}\right),\right. \\
& \left.\xi_{1}+\xi_{2}>2 K_{1}+2 K_{2}+2 K_{f}\right\} \\
& \Omega_{3}=\left\{\xi_{2}>2 K_{2}, \quad \alpha_{1} \xi_{2}-\alpha_{2} \xi_{1}<2 \alpha_{1} K_{2}-2 \alpha_{2}\left(K_{1}+K_{f}\right)\right\} \\
& \Omega_{4}=\left\{\xi_{1}>2 K_{1}, \quad \alpha_{1} \xi_{2}-\alpha_{2} \xi_{1}>-2 \alpha_{2} K_{1}+2 \alpha_{1}\left(K_{2}+K_{f}\right)\right\}
\end{aligned}
$$




$$
\begin{aligned}
& \Omega_{5}=\left\{\xi_{1}<2 K_{1}, \quad \xi_{2}>2 K_{2}+2 K_{f}\right\} \\
& \Omega_{6}=\left\{\xi_{1}>2 K_{1}+2 K_{f}, \quad \xi_{2}<2 K_{2}\right\},
\end{aligned}
$$

where "," corresponds to the logical operator "and."

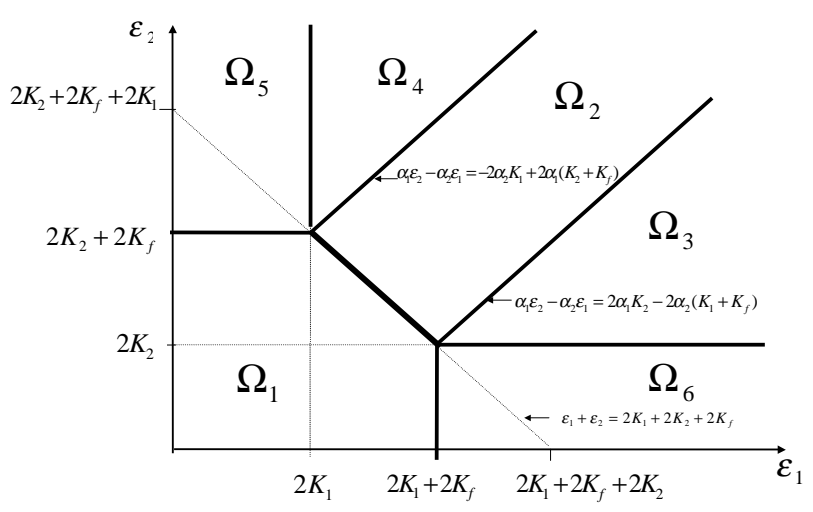

Figure 1: The demand space for $\left(\epsilon_{1}, \epsilon_{2}\right)$ in Stage 2

It is easy to show that function $\Pi$ is strictly jointly concave in $p_{i}, i=1,2$. Therefore, the first order KKT conditions are necessary and sufficient for optimality and any optimal solution is unique in $p_{i}, i=1,2$. Using these properties, given realizations $\epsilon_{1}$ and $\epsilon_{2}$ of random variables $\xi_{1}$ and $\xi_{2}$, the optimal solution value to Problem $P_{2}^{\prime}$ can be characterized in the following lemma.

Lemma 4.1 Given realizations $\epsilon_{1}$ and $\epsilon_{2}$ of random variables $\xi_{1}$ and $\xi_{2}$ and a resource 
investment vector $\vec{K}$, the optimal solution value to Problem $P_{2}^{\prime}$ can be expressed as:

$$
\Pi(\vec{K}, \vec{\epsilon})= \begin{cases}\sum_{i=1}^{2} \frac{\epsilon_{i}^{2}}{4 \alpha_{i}}, & \text { if } \Omega_{1} \\ \sum_{i=1}^{2}\left[\frac{\epsilon_{i}}{2 \alpha_{i}}+\frac{\epsilon_{1}+\epsilon_{2}-2\left(K_{1}+K_{2}+K_{f}\right)}{2\left(\alpha_{1}+\alpha_{2}\right)}\right] & \\ \left.\frac{\epsilon_{i}}{2}-\frac{\alpha_{i}\left(\epsilon_{1}+\epsilon_{2}-2\left(K_{1}+K_{2}+K_{f}\right)\right)}{2\left(\alpha_{1}+\alpha_{2}\right)}\right], & \text { if } \Omega_{2} \\ \frac{\left(\epsilon_{1}-K_{1}-K_{f}\right)\left(K_{1}+K_{f}\right)}{\alpha_{1}}+\frac{\left(\epsilon_{2}-K_{2}\right) K_{2}}{\alpha_{2}}, & \text { if } \Omega_{3} \\ \frac{\epsilon_{1}^{2}}{4 \alpha_{1}}+\frac{\left(\epsilon_{2}-K_{1}\right.}{\alpha_{1}-\frac{\left(\epsilon_{2}-K_{2}-K_{f}\right)\left(K_{2}+K_{2}+K_{f}\right)}{\alpha_{2}},}, & \text { if } \Omega_{4} \\ \frac{\left(\epsilon_{1}-K_{1}-K_{f}\right)\left(K_{1}+K_{f}\right)}{\alpha_{1}}+\frac{\epsilon_{2}^{2}}{4 \alpha_{2}}, & \text { if } \Omega_{5}\end{cases}
$$

Proof: See Appendix A.

Observe that $\Omega_{1}$ is the set of demand realizations where resource capacities are not constraining in the optimal solution, i.e., the unconstrained solution, $p_{i}^{(u)}=\frac{\epsilon_{i}}{2 \alpha_{i}}$, which induces a demand of $d_{i}^{(u)}=\frac{\epsilon_{i}}{2}, i=1,2$, is optimal. All other sets correspond to solutions on the boundary lines of the feasible region. $\Omega_{2}$ is the set whose optimal solution uses all resources fully and the flexible resource is shared by the two demand-classes with $d_{i}=$ $\frac{\epsilon_{i}}{2}-\frac{\alpha_{i}\left(\epsilon_{1}+\epsilon_{2}-2\left(K_{1}+K_{2}+K_{f}\right)\right)}{2\left(\alpha_{1}+\alpha_{2}\right)}, i=1,2 ; \Omega_{3}$ is the set where the amount of demand-class 1 satisfied is optimally set to $K_{1}+K_{f}$ and that of demand-class 2 to $K_{2}$; symmetrically, $\Omega_{4}$ is the set where the amount of demand-class 2 satisfied is $K_{2}+K_{f}$ and that of demand-class 1 is $K_{1} ; \Omega_{5}$ is the set where the amount of demand-class 2 satisfied is $K_{2}+K_{f}$ and that of demand-class 1 is the unconstrained $d_{1}^{(u)}=\frac{\epsilon_{1}}{2}$; and $\Omega_{6}$ is the set where the optimal amount of demand-class 1 satisfied is $K_{1}+K_{f}$ and that of demand-class 2 is the unconstrained 
$d_{2}^{(u)}=\frac{\epsilon_{2}}{2}$

Observe that as $\alpha_{2}$, the price elasticity of demand-class 2, increases, the region for set $\Omega_{3}$, corresponding to optimal allocation quantities of $\left(K_{1}+K_{f}, K_{2}\right)$, will expand, while the region for set $\Omega_{4}$, corresponding to optimal allocation quantities of $\left(K_{1}, K_{2}+K_{f}\right)$, will shrink. Thus, class 2 becomes less desirable to satisfy in the optimal solution.

This characterization of the optimal pricing and allocation decision extends the characterization in $\mathrm{FF}$ to one with continuous distributions for the random variables $\xi_{i}, i=1,2$. We also note here that this characterization extends the one in VM, since VM assumes that the firm is a price-taker, i.e., $p_{1}$ and $p_{2}$ are exogenously determined, whereas our model also incorporates the demand elasticities and pricing decision (in an environment with no competition) into the resource investment framework. 


\section{Chapter 5}

\section{Characterization of the Optimal}

\section{Investment Strategy in Stage 1}

Using the characterization of the optimal solution to the Stage 2 Problem, stated in Lemma 4.1, we can now analyze the structure of the optimal investment strategy. The firm seeks a coordinated strategy of investment, resource allocation, and pricing decision so as to maximize its expected profit in Stage 1, given by:

$$
\begin{aligned}
& \text { (Stage 1 Problem) } P_{1}: \quad \max _{\vec{K}} V(\vec{K})=E[\Pi(\vec{K}, \vec{\xi})]-\sum_{i=1,2, f} c_{i} K_{i} \\
& \text { subject to } \\
& K_{i} \geq 0, \quad i=1,2, f \quad \longleftarrow v_{i}
\end{aligned}
$$

Let $\vec{v}=\left(v_{1}, v_{2}, v_{f}\right) \geq 0$ represent the corresponding Lagrangian multipliers. We denote the maximizer of $V(\vec{K})$ by $\vec{K}^{*}$ and refer to $\vec{K}^{*}$ as the optimal investment vector. We have the following results.

Lemma $5.1 V(\vec{K})$ is strictly jointly concave in $K_{1}, K_{2}, K_{f}$ for any continuous distribution 
of $\xi_{1}$ and $\xi_{2}$ in $[0, \infty)$.

Proof: See Appendix B.

Thus, the optimal investment vector $\vec{K}^{*}$ is unique and the first-order KKT conditions are necessary and sufficient for optimality. These lead to the following theorem.

Theorem 5.1 An investment vector $\vec{K} \in R_{+}^{3}$ is optimal if and only if there exists a $\vec{v} \in R_{+}^{3}$ that satisfies the following conditions:

$$
\begin{aligned}
& \mathbf{E}\left(\begin{array}{c}
\frac{\xi_{1}+\xi_{2}-2 K_{T}}{\alpha_{1}+\alpha_{2}} \\
\frac{\xi_{1}+\xi_{2}-2 K_{T}}{\alpha_{1}+\alpha_{2}} \\
\frac{\xi_{1}+\xi_{2}-2 K_{T}}{\alpha_{1}+\alpha_{2}}
\end{array}\right) \operatorname{Pr}\left(\boldsymbol{\Omega}_{\mathbf{2}}\right)+\mathbf{E}\left(\begin{array}{c}
\frac{\xi_{1}-2 K_{1}-2 K_{f}}{\alpha_{1}} \\
\frac{\xi_{2}-2 K_{2}}{\alpha_{2}} \\
\frac{\xi_{1}-2 K_{1}-2 K_{f}}{\alpha_{1}}
\end{array}\right) \operatorname{\Omega _{3}} \operatorname{Pr}\left(\boldsymbol{\Omega}_{\mathbf{3}}\right) \\
& +\mathbf{E}\left(\begin{array}{c}
\frac{\xi_{1}-2 K_{1}}{\alpha_{1}} \\
\frac{\xi_{2}-2 K_{2}-2 K_{f}}{\alpha_{2}} \\
\frac{\xi_{2}-2 K_{2}-2 K_{f}}{\alpha_{2}}
\end{array}\right) \operatorname{Pr}\left(\boldsymbol{\Omega}_{\mathbf{4}}\right)+\mathbf{E}\left(\begin{array}{c}
0 \\
\frac{\xi_{2}-2 K_{2}-2 K_{f}}{\alpha_{2}} \\
\frac{\xi_{2}-2 K_{2}-2 K_{f}}{\alpha_{2}}
\end{array}\right) \operatorname{Pr}\left(\boldsymbol{\Omega}_{\mathbf{5}}\right) \\
& +\mathbf{E}\left(\begin{array}{c}
\frac{\xi_{1}-2 K_{1}-2 K_{f}}{\alpha_{1}} \\
0 \\
\frac{\xi_{1}-2 K_{1}-2 K_{f}}{\alpha_{1}}
\end{array}\right) \operatorname{Pr}\left(\boldsymbol{\Omega}_{\mathbf{6}}\right)=\left(\begin{array}{c}
c_{1}-v_{1} \\
c_{2}-v_{2} \\
c_{f}-v_{f}
\end{array}\right) \\
& v_{i} K_{i}=0, \quad i=1,2, f
\end{aligned}
$$

Theorem 5.1 extends Proposition 1 of VM to the case where prices are also decision variables. Based on this theorem, we characterize the firm's optimal investment strategy in the following. 
We first establish the necessary and sufficient conditions for investing in the flexible resource. For this purpose, we analyze the boundary solution $\vec{K}^{D}=\left(K_{1}^{D}>0, K_{2}^{D}>\right.$ $0, K_{f}^{D}=0$ ) (with the corresponding optimal Lagrangian multipliers being $v_{1}=0, v_{2}=0$, $v_{f} \geq 0$ ), where the firm only invests in the dedicated resources. We then use this solution to characterize the conditions for investing in the flexible resource. At solution $\vec{K}^{D}$, our demand space, given in Eq. (14), reduces to the following.

$$
\begin{aligned}
& \Omega_{1}^{D}=\left\{\xi_{1}<2 K_{1}^{D}, \quad \xi_{2}<2 K_{2}^{D}, \quad \xi_{1}+\xi_{2}<2 K_{1}^{D}+2 K_{2}^{D}\right\} \\
& =\left\{\xi_{1}<2 K_{1}^{D}, \quad \xi_{2}<2 K_{2}^{D}\right\} \\
& \Omega_{2}^{D}=\left\{\xi_{1}+\xi_{2}>2 K_{1}^{D}+2 K_{2}^{D}, \quad 2 \alpha_{1} K_{2}^{D}-2 \alpha_{2} K_{1}^{D}<\alpha_{1} \xi_{2}-\alpha_{2} \xi_{1}<2 \alpha_{1} K_{2}^{D}-2 \alpha_{2} K_{1}^{D}\right\} \\
& \Omega_{3}^{D}=\left\{\xi_{2}>2 K_{2}^{D}, \alpha_{1} \xi_{2}-\alpha_{2} \xi_{1}<2 \alpha_{1} K_{2}^{D}-2 \alpha_{2} K_{1}^{D}\right\} \\
& \Omega_{4}^{D}=\left\{\xi_{1}>2 K_{1}^{D}, \quad \alpha_{1} \xi_{2}-\alpha_{2} \xi_{1}>-2 \alpha_{2} K_{1}^{D}+2 \alpha_{1} K_{2}^{D}\right\} \\
& \Omega_{5}^{D}=\left\{\xi_{1}<2 K_{1}^{D}, \quad \xi_{2}>2 K_{2}^{D}\right\} \\
& \Omega_{6}^{D}=\left\{\xi_{1}>2 K_{1}^{D}, \quad \xi_{2}<2 K_{2}^{D}\right\}
\end{aligned}
$$

Observe that since $\xi_{i}, i=1,2$, are continuous random variables, $\Omega_{2}^{D}=\emptyset$, except for the special case where $\operatorname{Pr}\left(\alpha_{1} \xi_{2}=\alpha_{2} \xi_{1}\right)=1$ and $\alpha_{1} K_{2}^{D}=\alpha_{2} K_{1}^{D}$. In order to simplify the presentation, in the following we will consider that $\Omega_{2}^{D}=\emptyset$. All our results hold for this special case as well.

Thus, at boundary solution $\vec{K}^{D}$, the optimality conditions given in Theorem 5.1 reduce to the following.

Corollary 5.1 Boundary solution $\vec{K}^{D}=\left(K_{1}^{D} \geq 0, K_{2}^{D} \geq 0, K_{f}^{D}=0\right)$ is optimal if and 
only if there exists a $v_{f} \geq 0$ that satisfies the first-order KKT necessary and sufficient conditions, given below:

$$
\begin{aligned}
& \text { KKT - 1 : } \\
& E\left[\frac{\xi_{1}-2 K_{1}^{D}}{\alpha_{1}} \mid \Omega_{3}^{D}\right] \operatorname{Pr}\left(\Omega_{3}^{D}\right)+E\left[\frac{\xi_{1}-2 K_{1}^{D}}{\alpha_{1}} \mid \Omega_{4}^{D}\right] \operatorname{Pr}\left(\Omega_{4}^{D}\right)+E\left[\frac{\xi_{1}-2 K_{1}^{D}}{\alpha_{1}} \mid \Omega_{6}^{D}\right] \operatorname{Pr}\left(\Omega_{6}^{D}\right)=c_{1} \\
& \Rightarrow E\left[\xi_{1}-2 K_{1}^{D} \mid \xi_{1}>2 K_{1}^{D}\right] \operatorname{Pr}\left(\xi_{1}>2 K_{1}^{D}\right)=\alpha_{1} c_{1}
\end{aligned}
$$

\section{KKT -2 :}

$$
\begin{aligned}
& E\left[\frac{\xi_{2}-2 K_{2}^{D}}{\alpha_{2}} \mid \Omega_{3}^{D}\right] \operatorname{Pr}\left(\Omega_{3}^{D}\right)+E\left[\frac{\xi_{2}-2 K_{2}^{D}}{\alpha_{2}} \mid \Omega_{4}^{D}\right] \operatorname{Pr}\left(\Omega_{4}^{D}\right)+E\left[\frac{\xi_{2}-2 K_{2}^{D}}{\alpha_{2}} \mid \Omega_{5}^{D}\right] \operatorname{Pr}\left(\Omega_{5}^{D}\right)=c_{2} \\
& \Rightarrow E\left[\xi_{2}-2 K_{2}^{D} \mid \xi_{2}>2 K_{2}^{D}\right] \operatorname{Pr}\left(\xi_{2}>2 K_{2}^{D}\right)=\alpha_{2} c_{2}
\end{aligned}
$$

\section{KKT -3 :}

$$
\begin{aligned}
& E\left[\frac{\xi_{1}-2 K_{1}^{D}}{\alpha_{1}} \mid \Omega_{3}^{D}\right] \operatorname{Pr}\left(\Omega_{3}^{D}\right)+E\left[\frac{\xi_{2}-2 K_{2}^{D}}{\alpha_{2}} \mid \Omega_{4}^{D}\right] \operatorname{Pr}\left(\Omega_{4}^{D}\right)+E\left[\frac{\xi_{2}-2 K_{2}^{D}}{\alpha_{2}} \mid \Omega_{5}^{D}\right] \operatorname{Pr}\left(\Omega_{5}^{D}\right) \\
& +E\left[\frac{\xi_{1}-2 K_{1}^{D}}{\alpha_{1}} \mid \Omega_{6}^{D}\right] \operatorname{Pr}\left(\Omega_{6}^{D}\right)=c_{f}-v_{f} \\
& \Rightarrow \underline{c}_{f} \equiv c_{f}-v_{f}=\frac{1}{\alpha_{1}} E\left[\xi_{1}-2 K_{1}^{D} \mid A\right] \operatorname{Pr}(A)+\frac{1}{\alpha_{2}} E\left[\xi_{2}-2 K_{2}^{D} \mid B\right] \operatorname{Pr}(B),
\end{aligned}
$$

where $A=\left\{\xi_{1}>2 K_{1}^{D}, \alpha_{1} \xi_{2}-\alpha_{2} \xi_{1}<2 \alpha_{1} K_{2}^{D}-2 \alpha_{2} K_{1}^{D}\right\}$ and $B=\left\{\xi_{2}>2 K_{2}^{D}, \alpha_{1} \xi_{2}-\right.$ $\left.\alpha_{2} \xi_{1}>2 \alpha_{1} K_{2}^{D}-2 \alpha_{2} K_{1}^{D}\right\}$.

Observe that the first two KKT conditions in Corollary 5.1 also represent the optimality conditions in the dedicated system, when it is viable to invest in any capacity for each demand-class (see Lemma 3.1), and $\underline{c}_{f}$ is defined as a function of $K_{1}^{D}$ and $K_{2}^{D}$ in the third KKT condition. Recall that $\vec{K}^{I}$ denotes the optimal solution to the dedicated system. Thus, if solution $\left(K_{1}^{D}=K_{1}^{I}, K_{2}^{D}=K_{2}^{I}, K_{f}^{D}=0\right)$ also satisfies the third KKT condition in Eq. (18), then the optimal solution to the dedicated system is also optimal for the original 
problem and corresponds to boundary solution $\vec{K}^{D}$. These lead to the following lemma.

Lemma 5.2 The optimal investment strategy is such that $K_{f}^{*}>0$ only if $c_{f}<\underline{c}_{f}$, where $\underline{c}_{f}$ is defined in Equation (18).

Proof:

The proof follows directly from the KKT conditions at boundary solution $\vec{K}^{D}$. This solution is optimal if and only if its corresponding KKT conditions, given in Eq.s (16), (17), and (18), are satisfied with respect to a non-negative value of $v_{f}$. Observe that when $c_{f}<\underline{c}_{f}$, the KKT condition in Eq. (18) cannot be satisfied with respect to a $v_{f} \geq 0$, and hence, the boundary solution $\vec{K}^{D}=\left(K_{1}^{D}>0, K_{2}^{D}>0, K_{f}^{D}=0\right)$ can no longer be optimal. Thus, at optimality we must have $K_{f}^{*}>0$.

Thus, the flexible resource will be beneficial only when its unit investment cost is not too expensive. Lemma 5.2 extends Theorem 1 of FF to our multi-dimensional newsvendor model with pricing and has a similar interpretation. Recall that $\gamma_{i}^{I}$ is the optimal Lagrangian multiplier corresponding to the capacity constraint for the dedicated resource in the Stage 2 Problem of demand-class $i$ in the dedicated system. Using Eq.s (12) and (18), we can write:

$$
\begin{aligned}
\underline{c}_{f}= & E\left[\frac{\xi_{1}-2 K_{1}^{D}}{\alpha_{1}} \mid \Omega_{3}^{D}\right] \operatorname{Pr}\left(\Omega_{3}^{D}\right)+E\left[\frac{\xi_{1}-2 K_{1}^{D}}{\alpha_{1}} \mid \Omega_{6}^{D}\right] \operatorname{Pr}\left(\Omega_{6}^{D}\right) \\
& +E\left[\frac{\xi_{2}-2 K_{2}^{D}}{\alpha_{2}} \mid \Omega_{4}^{D}\right] \operatorname{Pr}\left(\Omega_{4}^{D}\right)+E\left[\frac{\xi_{2}-2 K_{2}^{D}}{\alpha_{2}} \mid \Omega_{5}^{D}\right] \operatorname{Pr}\left(\Omega_{5}^{D}\right) \\
= & E\left[\max _{i=1,2}\left\{\gamma_{i}^{I}\right\}\right],
\end{aligned}
$$


thus establishing the relationship between Theorem 1 of FF and our result. Hence, as stated in $\mathrm{FF}$, the firm invests in the flexible resource "when the expected value of its best usage exceeds its cost (page 450 in Fine and Freund (1990))."

We next develop insights into the firm's optimal investment decision when it includes the flexible resource. We first note that when $K_{f}^{*}>0$ in the optimal solution, the solution must be one of the following forms, each of which corresponds to a boundary solution of the feasible region for the Stage 1 Problem:

$$
\begin{aligned}
& \vec{K}^{F}=\left(K_{1}^{F}=0, \quad K_{2}^{F}=0, \quad K_{f}^{F}>0\right) \\
& \vec{K}^{1 F}=\left(K_{1}^{1 F}>0, \quad K_{2}^{1 F}=0, \quad K_{f}^{1 F}>0\right) \\
& \vec{K}^{2 F}=\left(K_{1}^{2 F}=0, \quad K_{2}^{2 F}>0, \quad K_{f}^{2 F}>0\right) \\
& \vec{K}^{A}=\left(K_{1}^{A}>0, \quad K_{2}^{A}>0, \quad K_{f}^{A}>0\right) .
\end{aligned}
$$

In what follows, we let $\Omega_{j}^{F}, \Omega_{j}^{1 F}, \Omega_{j}^{2 F}$, and $\Omega_{j}^{A}$ respectively denote set $\Omega_{j}, j=1, \cdots, 6$, given in Equation (14), at boundary solutions $\vec{K}^{F}, \vec{K}^{1 F}, \vec{K}^{2 F}$, and $\vec{K}^{A}$.

Lemma 5.3 Consider the boundary solutions $\vec{K}^{F}, \vec{K}^{1 F}, \vec{K}^{2 F}$, and $\vec{K}^{A}$. We have the following properties:

1. $\vec{K}^{F}=\left(K_{1}^{F}=0, K_{2}^{F}=0, K_{f}^{F}>0\right)$ is not a possible solution if $\left\{\left(\Omega_{3}^{F}=\emptyset\right)\right.$ or $\left.\left(\Omega_{4}^{F}=\emptyset\right)\right\}$

2. $\vec{K}^{1 F}=\left(K_{1}^{1 F}>0, K_{2}^{1 F}=0, K_{f}^{1 F}>0\right)$ is not a possible solution if $\left\{\left(\Omega_{3}^{1 F}=\emptyset\right)\right.$ or $\left.\left(\Omega_{4}^{1 F}=\emptyset, \Omega_{5}^{1 F}=\emptyset\right)\right\}$

3. $\vec{K}^{2 F}=\left(K_{1}^{2 F}=0, K_{2}^{2 F}>0, K_{f}^{2 F}>0\right)$ is not a possible solution if $\left\{\left(\Omega_{4}^{2 F}=\emptyset\right)\right.$ or 
$\left.\left(\Omega_{3}^{2 F}=\emptyset, \Omega_{6}^{2 F}=\emptyset\right)\right\}$

4. $\vec{K}^{A}=\left(K_{1}^{A}>0, K_{2}^{A}>0, K_{f}^{A}>0\right)$ is not a possible solution if $\left\{\left(\Omega_{4}^{A}=\emptyset, \Omega_{5}^{A}=\emptyset\right)\right.$ $\left.\operatorname{or}\left(\Omega_{3}^{A}=\emptyset, \Omega_{6}^{A}=\emptyset\right)\right\}$.

Proof: See Appendix C.

Lemma 5.3 leads to the following result.

Theorem 5.2 Consider the case where $\operatorname{Pr}\left(\frac{\xi_{1}}{\alpha_{1}}<\frac{\xi_{2}}{\alpha_{2}}\right)=1$, that is, $\alpha_{2} \xi_{1}<\alpha_{1} \xi_{2}$ with probability 1. Then, if $c_{f}<\underline{c}_{f}$, the optimal strategy must be one of the following forms:

1. Invest in dedicated resource 2 and the flexible resource only (boundary solution $\vec{K}^{2 F}$ ). In this case, $K_{2}^{2 F}>\frac{\alpha_{2}}{\alpha_{1}} K_{f}^{2 F}$; or

2. Invest in all three resources (boundary solution $\left.\vec{K}^{A}\right)$. In this case, $K_{2}^{A}>\frac{\alpha_{2}}{\alpha_{1}}\left(K_{1}^{A}+\right.$ $\left.K_{f}^{A}\right)$

Proof:

Consider that $\operatorname{Pr}\left(\frac{\xi_{1}}{\alpha_{1}}<\frac{\xi_{2}}{\alpha_{2}}\right)=1$. Since $\alpha_{1} \xi_{2}-\alpha_{2} \xi_{1}>0$ with probability $1, \Omega_{3}^{F}=$ $\left\{\alpha_{1} \xi_{2}-\alpha_{2} \xi_{1}<-2 \alpha_{2} K_{f}^{F}\right\}=\emptyset$ for any $K_{f}^{F}>0$, where $\Omega_{3}^{F}$ is obtained by setting $K_{f}^{F}>0$, $K_{1}^{F}=0, K_{2}^{F}=0$ in $\Omega_{3}$, given in Eq. (14). Thus, it follows, by Lemma 5.3 , that $\vec{K}^{F}$ is not a possible solution in this case. Similarly, $\Omega_{3}^{1 F}=\left\{\alpha_{1} \xi_{2}-\alpha_{2} \xi_{1}<-2 \alpha_{2}\left(K_{1}^{1 F}+K_{f}^{1 F}\right)\right\}=\emptyset$ for any $K_{1}^{1 F}>0$ and $K_{f}^{1 F}>0$, and hence $\vec{K}^{1 F}$ is not a possible solution by Lemma 5.3. Thus, when $c_{f}<\underline{c}_{f}$, Lemma 5.2 implies that $K_{f}^{*}>0$ in the optimal solution, which must correspond to either boundary solution $\vec{K}^{2 F}$ or $\vec{K}^{A}$. 
Observe that by Lemma $5.3, \vec{K}^{A}$ is a possible solution only when $\left\{\left(\Omega_{4}^{A} \neq \emptyset\right.\right.$ or $\Omega_{5}^{A} \neq$ $\emptyset)$ and $\left(\Omega_{3}^{A} \neq \emptyset\right.$ or $\left.\left.\Omega_{6}^{A} \neq \emptyset\right)\right\}$. We can write the second condition as:

$$
\begin{aligned}
& \left(\Omega_{3}^{A} \neq \emptyset \text { or } \Omega_{6}^{A} \neq \emptyset\right)=\left\{\left(\Omega_{3}^{A} \text { or } \Omega_{6}^{A}\right) \neq \emptyset\right\} \\
= & \left\{\xi_{1}>2 K_{1}^{A}+2 K_{f}^{A}, \alpha_{1} \xi_{2}-\alpha_{2} \xi_{1}<2 \alpha_{1} K_{2}^{A}-2 \alpha_{2}\left(K_{1}^{A}+K_{f}^{A}\right)\right\} \neq \emptyset,
\end{aligned}
$$

(see Eq. (14) and Figure 1)

or equivalently, $\operatorname{Pr}\left\{\xi_{1}>2 K_{1}^{A}+2 K_{f}^{A}, \alpha_{1} \xi_{2}-\alpha_{2} \xi_{1}<2 \alpha_{1} K_{2}^{A}-2 \alpha_{2}\left(K_{1}^{A}+K_{f}^{A}\right)\right\} \neq 0$. Since $\alpha_{1} \xi_{2}-\alpha_{2} \xi_{1}>0$ with probability 1 , we must have $2 \alpha_{1} K_{2}^{A}-2 \alpha_{2}\left(K_{1}^{A}+K_{f}^{A}\right)>0$. Thus, $K_{2}^{A}>\frac{\alpha_{2}}{\alpha_{1}}\left(K_{1}^{A}+K_{f}^{A}\right)$, if $\vec{K}^{A}$ is the optimal solution. By a similar argument, we can show that if $\vec{K}^{2 F}$ is the optimal solution, then it must satisfy $K_{2}^{2 F}>\frac{\alpha_{2}}{\alpha_{1}} K_{f}^{2 F}$. This completes the proof.

Consider the Stage 2 Problem, $P_{2}^{\prime}$. If resource levels were unconstraining, then the price for demand-class $i, i=1,2$, would be optimally set to $p_{i}^{(u)}=\frac{\epsilon_{i}}{2 \alpha_{i}}$. Thus, when $\operatorname{Pr}\left(\frac{\xi_{1}}{\alpha_{1}}<\frac{\xi_{2}}{\alpha_{2}}\right)=1$, we have $p_{1}^{(u)}<p_{2}^{(u)}$; that is, demand-class 2 would be priced higher than demand-class 1 if resource capacities were not constraining. In other words, demand-class 2 is the more desirable demand-class to satisfy. Theorem 5.2 states that in this case the optimal investment strategy gives higher priority to resources that can satisfy demand-class 2: If a flexible resource investment is made, then we either invest in dedicated resource 2 and the flexible resource only (solution $\vec{K}^{2 F}$ ); or we invest in all three resources (solution $\vec{K}^{A}$ ). No other solution can be optimal. Furthermore, the optimal level of dedicated resource 2 is greater than the combined optimal capacities of the other resources (flexible resource plus the dedicated resource of the other demand-class) times its relative price 
elasticity $\left(\alpha_{2} / \alpha_{1}\right)$. This result, together with Lemma 5.2, extends Proposition 2 in VM, who shows that the optimal investment strategy, when prices are exogenously determined, is to invest either in dedicated resources only, or in the dedicated resource for the higher priced demand-class and the flexible resource, or in all three resources.

We also want to point out that when $\alpha_{1}=\alpha_{2}$, that is, when both demand-classes have the same price elasticity of demand, the condition that $\operatorname{Pr}\left(\frac{\xi_{1}}{\alpha_{1}}<\frac{\xi_{2}}{\alpha_{2}}\right)=1$ reduces to the condition that $\operatorname{Pr}\left(\xi_{1}<\xi_{2}\right)=1$. In this case, the demand curve of demand-class 2 dominates that of demand-class 1 over the entire range of prices; that is, the same demand level corresponds to a higher price for demand-class 2 over demand-class 1, or equivalently, the same price induces a higher demand for demand-class 2. Theorem 5.2 shows that in such cases, the optimal strategy will invest more in dedicated resource 2, thus translating into higher revenues. That is, if solution $\vec{K}^{2 F}$ is optimal, then $K_{2}^{2 F}>K_{f}^{2 F}$; if solution $\vec{K}^{A}$ is optimal, then $K_{2}^{A}>K_{1}^{A}+K_{f}^{A}$. 


\section{Chapter 6}

\section{Impact of Demand Correlation on the}

\section{Optimal Investment Strategy}

In the following, we study the impact of demand correlation on the optimal investment strategy and show that it might be optimal to invest in the flexible resource even when the demand patterns for the two demand-classes are perfectly positively correlated, whereas it might be optimal not to invest in the flexible resource when demand patterns are perfectly negatively correlated. In order to avoid the trivial cases and simplify the presentation, in the following we consider the case where it is economically viable to invest in any capacity for each demand-class; that is, we consider that $c_{i}<\frac{E\left[\xi_{i}\right]}{\alpha_{i}}, i=1,2$ (see Lemma 3.1).

Let $\rho$ denote the correlation coefficient between $\xi_{1}$ and $\xi_{2}$. As in the previous section, we do not make any distributional assumptions on $\xi_{1}$ and $\xi_{2}$. All the following results hold for any continuous distribution of $\xi_{1}$ and $\xi_{2}$ defined over $[0, \infty)$. 


\subsection{Perfectly Positively Correlated Demand Patterns}

We first analyze the case where the demand patterns are perfectly positively correlated. For this purpose, we consider that $\operatorname{Pr}\left(\xi_{1}=a \xi_{2}\right)=1$, for some constant $a>0$; that is, $\rho=+1$. For the sake of simplicity in notation, let $\xi_{2}=\xi$, and thus $\xi_{1}=a \xi$.

In this case, the first two optimality conditions at boundary solution $\vec{K}^{D}=\left(K_{1}^{D}>\right.$ 0, $K_{2}^{D}>0, K_{f}^{D}=0$ ), given in Eq.s (16) and (17), reduce to the following:

$$
\begin{gathered}
E\left[\xi-\frac{2 K_{1}^{D}}{a} \mid \xi>\frac{2 K_{1}^{D}}{a}\right] \operatorname{Pr}\left(\xi>\frac{2 K_{1}^{D}}{a}\right)=\frac{\alpha_{1} c_{1}}{a} \\
E\left[\xi-2 K_{2}^{D} \mid \xi>2 K_{2}^{D}\right] \operatorname{Pr}\left(\xi>2 K_{2}^{D}\right)=\alpha_{2} c_{2}
\end{gathered}
$$

As was done in Chapter 5 for the general case, we first study the characteristics of the boundary solution $\vec{K}^{D}$, which corresponds to the case where the firm does not invest in the flexible resource.

Lemma 6.1 Let the demand patterns for the two demand-classes be perfectly positively correlated; that is, $\operatorname{Pr}\left(\xi_{1}=a \xi_{2}\right)=1$, for some constant $a>0$, and hence $\rho=+1$. If boundary solution $\vec{K}^{D}=\left(K_{1}^{D}>0, K_{2}^{D}>0, K_{f}^{D}=0\right)$ is optimal, then it must have the following structure:

1. If $\frac{\alpha_{1} c_{1}}{a}>\alpha_{2} c_{2}$, then $\frac{K_{1}^{D}}{a}<K_{2}^{D}$;

2. If $\frac{\alpha_{1} c_{1}}{a}=\alpha_{2} c_{2}$, then $\frac{K_{1}^{D}}{a}=K_{2}^{D}$;

3. If $\frac{\alpha_{1} c_{1}}{a}<\alpha_{2} c_{2}$, then $\frac{K_{1}^{D}}{a}>K_{2}^{D}$.

Proof: The proof directly follows from the optimality conditions in Eq.s (19) and (20). 
Now we can characterize the optimal investment strategy in the case of perfectly positively correlated demands. We first analyze the conditions under which we invest in the flexible resource in the following lemma.

Lemma 6.2 Consider the case where $\operatorname{Pr}\left(\xi_{1}=a \xi_{2}\right)=1$, for some constant $a>0$. In the optimal strategy, we invest in the flexible resource $\left(K_{f}^{*}>0\right)$ only if $\left\{\left(\frac{\alpha_{1} c_{1}}{a}>\alpha_{2} c_{2}\right.\right.$ and $\alpha_{1}>a \alpha_{2}$ and $\left.c_{f}<\underline{c}_{f}\right)$ or $\left(\frac{\alpha_{1} c_{1}}{a}<\alpha_{2} c_{2}\right.$ and $\alpha_{1}<a \alpha_{2}$ and $\left.\left.c_{f}<\underline{c}_{f}\right)\right\}$.

\section{Proof: See Appendix D.}

Our main result in the case of perfectly positively correlated demand patterns is given in the following theorem.

Theorem 6.1 Consider the case where $\operatorname{Pr}\left(\xi_{1}=a \xi_{2}\right)=1$, for some constant $a>0$; that is, the two demand patterns are perfectly positively correlated. The optimal capacity investment decision has the following structure:

1. If $\left\{\frac{\alpha_{1} c_{1}}{a}>\alpha_{2} c_{2}\right.$ and $\left.\alpha_{1} \leq a \alpha_{2}\right\}$ or $\left\{\frac{\alpha_{1} c_{1}}{a}=\alpha_{2} c_{2}\right\}$ or $\left\{\frac{\alpha_{1} c_{1}}{a}<\alpha_{2} c_{2}\right.$ and $\left.\alpha_{1} \geq a \alpha_{2}\right\}$, then the optimal strategy is always to invest in dedicated resources only, such that

$$
\begin{aligned}
& \text { - If } \frac{\alpha_{1} c_{1}}{a}>\alpha_{2} c_{2} \text {, then } \frac{K_{1}^{D}}{a}<K_{2}^{D} ; \\
& \text { - If } \frac{\alpha_{1} c_{1}}{a}=\alpha_{2} c_{2} \text {, then } \frac{K_{1}^{D}}{a}=K_{2}^{D} ; \text { and } \\
& \text { - If } \frac{\alpha_{1} c_{1}}{a}<\alpha_{2} c_{2} \text {, then } \frac{K_{1}^{D}}{a}>K_{2}^{D} \text {. }
\end{aligned}
$$

2. If $\left\{\frac{\alpha_{1} c_{1}}{a}>\alpha_{2} c_{2}\right.$ and $\left.\alpha_{1}>a \alpha_{2}\right\}$, then

- If $c_{f}>\underline{c}_{f}$, then we invest in dedicated resources only $\left(\vec{K}^{D}\right)$; 
- If $c_{f}<\underline{c}_{f}$, then the optimal solution is either to (1) invest only in dedicated resource 2 and the flexible resource $\left(\vec{K}^{2 F}\right)$; or (2) invest in all three resources $\left(\vec{K}^{A}\right)$. Furthermore,

(a) If strategy $\vec{K}^{2 F}$ is optimal, then $\frac{K_{1}^{I}}{a}<\frac{K_{f}^{2 F}}{a}<K_{2}^{2 F}<K_{2}^{I}$;

(b) If strategy $\vec{K}^{A}$ is optimal, then $\frac{K_{1}^{A}}{a}<\frac{K_{1}^{I}}{a}<\frac{K_{1}^{A}+K_{f}^{A}}{a}<K_{2}^{A}<K_{2}^{I}<$ $K_{2}^{A}+K_{f}^{A}$, where $\vec{K}^{I}$ denotes the optimal solution to the dedicated system.

3. Symmetrically, if $\left\{\frac{\alpha_{1} c_{1}}{a}<\alpha_{2} c_{2}\right.$ and $\left.\alpha_{1}<a \alpha_{2}\right\}$, then

- If $c_{f}>\underline{c}_{f}$, then we invest in dedicated resources only $\left(\vec{K}^{D}\right)$;

- If $c_{f}<\underline{c}_{f}$, then the optimal solution is either to (1) invest only in dedicated resource 1 and the flexible resource $\left(\vec{K}^{1 F}\right)$; or (2) invest in all three resources $\left(\vec{K}^{A}\right)$. Furthermore,

(a) If strategy $\vec{K}^{1 F}$ is optimal, then $K_{2}^{I}<K_{f}^{1 F}<\frac{K_{1}^{1 F}}{a}<\frac{K_{1}^{I}}{a}$;

(b) If strategy $K^{A}$ is optimal, then $K_{2}^{A}<K_{2}^{I}<K_{2}^{A}+K_{f}^{A}<\frac{K_{1}^{A}}{a}<\frac{K_{1}^{I}}{a}<$ $\frac{K_{1}^{A}+K_{f}^{A}}{a}$.

Proof.

Part (1) follows from Lemmas 6.1 and 6.2. We now prove part (2). When $c_{f}<\underline{c}_{f}$, it follows, by Lemma 6.2, that $K_{f}^{*}>0$ in the optimal solution. Thus, in this case, the optimal strategy must correspond to one of the boundary solutions $\vec{K}^{F}, \vec{K}^{1 F}, \vec{K}^{2 F}$, or $\vec{K}^{A}$, each of which is analyzed below for the case where $\frac{\alpha_{1} c_{1}}{a}>\alpha_{2} c_{2}, \alpha_{1}>a \alpha_{2}$.

$$
\vec{K}^{F}=\left\{K_{1}^{F}=0, K_{2}^{F}=0, K_{f}^{F}>0\right\}
$$


When $\xi_{1}=a \xi_{2}, \Omega_{3}^{F}$, at boundary solution $\vec{K}^{F}$, reduces to

$$
\Omega_{3}^{F}=\{\xi \underbrace{\left(\alpha_{1}-a \alpha_{2}\right)}_{>0}<\underbrace{-2 \alpha_{2} K_{f}^{F}}_{<0}\}=\emptyset .
$$

Thus, by Lemma $5.3, \vec{K}^{F}$ is not a possible solution in this case.

$$
\underline{\vec{K}^{1 F}=\left\{K_{1}^{1 F}>0, K_{2}^{1 F}=0, K_{f}^{1 F}>0\right\}}
$$

In this case, $\Omega_{3}^{1 F}$, at boundary solution $\vec{K}^{1 F}$, reduces to

$$
\Omega_{3}^{1 F}=\{\xi \underbrace{\left(\alpha_{1}-a \alpha_{2}\right)}_{>0}<\underbrace{-2 \alpha_{2}\left(K_{1}^{1 F}+K_{f}^{1 F}\right)}_{<0}\}=\emptyset .
$$

Thus, by Lemma $5.3, \vec{K}^{1 F}$ is not a possible solution.

On the other hand, we can show, in a similar way, that boundary solutions $\vec{K}^{2 F}$ and $\vec{K}^{A}$ are possible. Consequently, when $\left\{\frac{\alpha_{1} c_{1}}{a}>\alpha_{2} c_{2}, \alpha_{1}>a \alpha_{2}, c_{f}<\underline{c}_{f}\right\}$, the optimal solution must be either $\vec{K}^{2 F}$ or $\vec{K}^{A}$.

Proofs of (2a) and (2b): See Appendix E.

The proof of part (3) is in the same spirit as the proof of part (2), and follows due to Lemma 5.3, since $\Omega_{4}^{F}=\emptyset$ (corresponding to boundary solution $\vec{K}^{F}$ ) and $\Omega_{4}^{2 F}=\emptyset$ (corresponding to boundary solution $\vec{K}^{2 F}$ ) when $\alpha_{1}<a \alpha_{2}$. Finally, proofs for parts (3a) and (3b) are also similar to that of part (2), and therefore, are omitted.

Theorem 6.1 states that if $c_{f}<\underline{c}_{f}$, then the firm should invest in the flexible resource 
only when either (i) $\frac{\alpha_{1} c_{1}}{a}>\alpha_{2} c_{2}, \alpha_{1}>a \alpha_{2}$ or (ii) $\frac{\alpha_{1} c_{1}}{a}<\alpha_{2} c_{2}, \alpha_{1}<a \alpha_{2}$.

Consider the first condition, $\frac{\alpha_{1} c_{1}}{a}>\alpha_{2} c_{2}, \alpha_{1}>a \alpha_{2}$. This condition implies that (1) the firm would charge a higher price for demand-class 2 than demand-class 1 if resource capacities were not constraining; that is, $p_{2}^{(u)}=\frac{\epsilon}{2} \frac{1}{\alpha_{2}}>p_{1}^{(u)}=\frac{\epsilon}{2} \frac{a}{\alpha_{1}}$; and (2) the dedicated resource investment cost per unconstrained price ratio corresponding to demand-class 2 is lower than that for demand-class 1 ; that is, $\frac{c_{2}}{1 / \alpha_{2}}<\frac{c_{1}}{a / \alpha_{1}}$. Thus, demand-class 2 is the "preferred" demand-class to satisfy. As a result, the optimal strategy gives higher priority to resources that can satisfy demand-class 2: If a flexible resource investment is made, then the firm invests in either dedicated resource 2 and the flexible resource only (boundary solution $\vec{K}^{2 F}$ ) or all three resources (boundary solution $\vec{K}^{A}$ ); no other solution can be optimal. The second condition is symmetric and corresponds to the case where demand-class 1 is preferred. Thus, Theorem 6.1 shows that in the case of perfectly positively correlated demand patterns $(\rho=+1)$, the flexible resource can still be valuable, but only when one demand-class is preferred, where the definition of preferred depends on demand function parameters and dedicated resource investment costs. If no demandclass is preferred, then the optimal solution does not invest in the flexible resource. Thus, our result nicely generalizes the result in VM, who shows that under exogenously fixed prices, flexible resource investment in the case of perfect positive correlation requires a price differential between the demand-classes.

Theorem 6.1 also provides insights on the substitution effect of the flexible resource. It shows that if it is optimal to invest in dedicated resource 2 and the flexible resource only (solution $\vec{K}^{2 F}$ ), then the structure of the optimal strategy is such that $K_{1}^{I}<K_{f}^{2 F}$ 
and $K_{2}^{2 F}<K_{2}^{I}$; that is, the flexible resource substitutes dedicated resource 1 in the dedicated system (solution $\vec{K}^{I}$ ) and is larger, while less of dedicated resource 2 is invested in, compared to solution $\vec{K}^{I}$ (part 2(a) of the theorem). Similarly, if it is optimal to invest in all resources (solution $\vec{K}^{A}$ ), then the firm invests less in each dedicated resource, while the total resource levels that can satisfy each demand-class are both higher $\left(K_{i}^{A}<K_{i}^{I}<K_{i}^{A}+K_{f}^{A}, i=1,2\right)$, compared to the optimal solution to the dedicated system (part 2(b) of the theorem).

Consider next the special case where $a=1$; that is, $\operatorname{Pr}\left(\xi_{1}=\xi_{2}\right)=1$. Then, the first condition reduces to $\alpha_{1} c_{1}>\alpha_{2} c_{2}, \alpha_{1}>\alpha_{2}$. In this case, the demand curve of class 2 dominates that of class 1 over all possible prices, since the same demand level corresponds to a higher price for demand-class 2 over demand-class 1 . In addition, the cost per unconstrained price ratio for class 2 is lower than that of class 1 . Then, the optimal investment strategy gives priority to resources that can satisfy demand-class 2 . In addition, the firm always invests more in dedicated resource 2 than the combined levels of the other resources (i.e., if solution $\vec{K}^{D}$ is optimal, then $K_{1}^{D}<K_{2}^{D}$; if solution $\vec{K}^{2 F}$ is optimal, then $K_{f}^{2 F}<K_{2}^{2 F}$; if solution $\vec{K}^{A}$ is optimal, then $\left.K_{1}^{A}+K_{f}^{A}<K_{2}^{A}\right)$.

\subsection{Perfectly Negatively Correlated Demand Patterns}

Next we analyze the case where demand patterns are perfectly negatively correlated. For this purpose, we consider that $\operatorname{Pr}\left(\xi_{1}+\xi_{2}=a\right)=1$ for some constant $a>0$, and hence $\rho=-1$. Thus, the sum of the random variables $\xi_{1}$ and $\xi_{2}$ is known with certainty, but the split between the two demand-classes is uncertain in Stage 1. Our main result in the case of perfect negative correlation is presented in the following theorem. 
Theorem 6.2 Consider the case where $\operatorname{Pr}\left(\xi_{1}+\xi_{2}=a\right)=1$, for some constant $a>0$; that is, the two demand patterns are perfectly negatively correlated $(\rho=-1)$. Then, there exists a threshold value, $\Delta^{\text {th }}$, such that:

1. If $K_{1}^{I}+K_{2}^{I} \geq \frac{a}{2}$, or equivalently if $\alpha_{2} c_{2}-\alpha_{1} c_{1} \leq \Delta^{\text {th }}$, then $K_{f}^{*}>0$ in the optimal solution, regardless of the value of $c_{f}$ (in the range $0<c_{1}, c_{2}<c_{f}<c_{1}+c_{2}$ ).

2. If $K_{1}^{I}+K_{2}^{I}<\frac{a}{2}$, or equivalently if $\alpha_{2} c_{2}-\alpha_{1} c_{1}>\Delta^{\text {th }}$, then

- if $c_{f}<\underline{c}_{f}$, then $K_{f}^{*}>0$ in the optimal solution;

- if $c_{f}>\underline{c}_{f}$, then $K_{f}^{*}=0$ in the optimal solution and the optimal solution is given by $\vec{K}^{D}$.

Proof: See Appendix F.

Thus, Theorem 6.2 shows that if the total capacity investment level in the dedicated system is high (i.e., $K_{1}^{I}+K_{2}^{I} \geq \frac{a}{2}$ ), then the optimal solution always invests in the flexible resource, regardless of the investment cost of the flexible resource, in the range considered. However, when the total capacity investment level in the dedicated system is lower (i.e., $\left.K_{1}^{I}+K_{2}^{I}<\frac{a}{2}\right)$, then it is not always optimal to invest in the flexible resource. This depends on the investment cost of the flexible resource. 


\section{Chapter 7}

\section{Conclusions and Future Research}

\section{Directions}

Resource flexibility can provide a competitive advantage to a firm by hedging against the demand uncertainty inherent in the capacity planning stage in most industries. However, flexible resources are generally more expensive than dedicated resources and the research that studies the value of resource flexibility is rather limited in the operations management/operations research literature. As a result, the utilization of flexible resources has been, so far, limited in industry. However, increasing competition in today's highly uncertain market place is forcing companies to look for strategies to more effectively match their supply with demand. Consequently, there is a great need to understand the value of resource flexibility and the factors that impact it. The value of resource flexibility depends on the firm's pricing strategy, demand characteristics, and correlations between the different demand-classes offered by the firm. Therefore, these factors need to be considered while studying the value of resource flexibility.

The resource investment decision in many industries requires long lead times and 
economies of scale in investment costs. As a result, this decision needs to be made under high demand uncertainty, and is difficult and expensive to change later on. Examples include investments in plant capacities or service capacities. Timing of these events naturally leads to the formulation of the decision problem as a two-stage stochastic decision problem with recourse, as is commonly done in the operations management literature. Our objective is to derive structural properties of the firm's optimal resource investment strategy and to understand how it is affected by the firm's pricing strategy, demand characteristics, and correlations. For this purpose, we make several simplifying assumptions so that the resulting models are amenable to analytical analysis. In that sense, our approach is highly stylized to serve as a practical decision support system, which may need to consider multi-period models with time-varying demand patterns and multiple resource types (such as plants, people, etc.). For such models, one generally must resort to numerical analysis, as has been done in, for instance, Caulkins and Fine (1990) and Li and Tirupati (1994).

As a result, we consider a simple two demand-class model, since this model is analytically tractable, while capturing the essential elements of the problem. We study the firm's optimal resource investment strategy, while incorporating the resource flexibility and the firm's pricing strategy into the investment decision. We characterize the structure of the firm's optimal resource investment strategy and use these results to understand the conditions under which the firm invests in the flexible resource, considering investment costs and demand parameters. We show that when one of the demand-classes is preferred to satisfy over the other one, then the optimal investment strategy gives a higher priority to resources that can satisfy the preferred demand-class. Specifically, if a flexible resource investment is made, then the firm either invests in the dedicated resource for the preferred 
demand-class and the flexible resource only, or in all three resources; no other solution can be optimal.

In addition, studying demand patterns that are perfectly positively correlated and perfectly negatively correlated, we are able to identify the conditions under which the firm invests in the flexible resource. Specifically, we are able to show that the firm can invest in the flexible resource even when demand patterns are perfectly positively correlated, under the condition that one demand-class is preferred over the other one, where the definition of preferred depends on demand function parameters and dedicated resource investment costs. If no demand-class is preferred, then the optimal solution does not invest in the flexible resource. Thus, our result nicely generalizes the result in VM, who shows that under exogenously fixed prices, flexible resource investment in the case of perfect positive correlation requires a price differential between the demand-classes. In addition, our analysis shows that when demand patterns are perfectly negatively correlated, it is not always optimal for the firm to invest in the flexible resource. This depends on the investment cost of the flexible resource. Thus, our results provide new insights into the value of resource flexibility under perfectly positively and perfectly negatively correlated demand patterns.

Our model and results contribute to the literature in several ways. First, we extend the well-known newsvendor problem by incorporating multiple suppliers, multiple demand types, and the pricing decision into the framework. Second, we characterize the structure of the firm's optimal investment strategy under the existence of flexible resources. Third, we study the impact of demand correlation on the firm's optimal resource investment strategy and provide new insights. 
Numerous extensions of our model deserve further analysis. Our study considered demand patterns that are perfectly positively and perfectly negatively correlated. These results need to be extended to any correlation coefficient to get a complete characterization of the impact of demand correlations on the value of resource flexibility. Other important extensions include analyzing different variable costs for the flexible and dedicated resources, different investment cost structures (including concave investment costs for the resources), and different demand functions.

In this thesis, we considered that the firm can utilize an integrated resource flexibility/price postponement strategy in the second stage to match its supply with demand. The next step would be to analyze the impact of the postponed pricing decision on the value of resource flexibility. We also assumed that the demand-classes are not substitutable with each other; that is, unsatisfied consumers will be lost. An interesting direction would be to consider the value of resource flexibility, considering that consumers might also substitute the demand-classes with each other, based on the firm's pricing strategy. 


\section{References}

BASSOK, Y. , R. ANUPINDI, AND R. AKELLA. 1999. Single-period Multiproduct Inventory Models with Substitution. Operations Research. 47, 632-642.

BAZARAA, M. S., H. D. SHERALI, AND C. M. SHETTY. 1993. Nonlinear Programming:

Theory and Algorithms. 2nd Ed., John Wiley \& Sons, Inc., New York.

BIRGE, J. R., J. DROGOSZ, AND I. DUENYAS. 1998. Setting Single-Period Optimal

Capacity Levels and Prices for Substitutable Products. The International Journal of Flexible Manufacturing Systems. 10, 407-430.

BISH, E. K., A. MURIEL, AND S. BILLER. 2001. Managing Flexible Capacity in a Make-to-Order Environment. Submitted to Management Science.

CAULKINS, J. P., AND C. H. FINE. 1990. Seasonal Inventories and the Use of ProductFlexible Manufacturing Technology. Annals of Operations Research. 26, 351-375.

EBERLY, J. C., AND J. A. VAN MIEGHEM. 1997. Multi-factor Dynamic Investment Under Uncertainty. Journal of Economic Theory. 75, 345-387.

FINE, C. H., AND R. M. FREUND. 1990. Optimal Investment in Product-Flexible Manufacturing Capacity. Management Science. 36, 449-466. 
HARRISON, J. M., AND J. A. VAN MIEGHEM. 1999. Multi-resource Investment Strategies: Operational Hedging under Demand Uncertainty. European Journal of Operational Research. 113, 17-29.

JORDAN, W. C., AND S. C. GRAVES. 1995. Principles and Benefits of Manufacturing Process Flexibility. Management Science. 41, 577-594.

LI, S. and D. TIRUPATI. 1994. Dynamic Capacity Expansion Problem with Multiple Products: Technology Selection and Timing of Capacity Additions. Operations Research. 42, 958-976.

LUSS, H. 1982. Operations Research and Capacity Expansion Problems: A Survey. Operations Research. 30, 907-947.

NETESSINE, S., G. DOBSON, AND R. A. SHUMSKY. 2002. Flexible Service Capacity: Optimal Investment and the Impact of Demand Correlation. Operations Research. 50, $375-388$.

PETRUZZI, N. C., AND M. DADA. 1999. Pricing and the Newsvendor Model: A Review with Extensions. Operations Research. 47, 183-194.

PORTEUS, E. L. 1990. "Stochastic Inventory Theory" in Handbooks in Operations Research and Management Science. 2, Eds. D. P. Heyman and M. J. Sobel, Elsevier Science Publishers, North-Holland, 605-652.

VAN MIEGHEM, J. A. 1998. Investment Strategies for Flexible Resources. Management Science. 44, 1071-1078. 


\section{Appendix}

In the following, let $\Omega^{\cup}$ represent the universal set.

\section{A Proof of Lemma 4.1}

If a feasible solution $\left(p_{1}, p_{2}\right)$ is a maximizer to problem $P_{2}^{\prime}$, then the first order KKT necessary conditions require that there exists a vector $\left(\lambda_{1}, \lambda_{2}, \mu\right) \geq 0$ such that

$$
\begin{gathered}
\epsilon_{1}-2 \alpha_{1} p_{1}+\alpha_{1} \lambda_{1}+\alpha_{1} \mu=0 \Rightarrow p_{1}=\frac{\epsilon_{1}+\alpha_{1} \lambda_{1}+\alpha_{1} \mu}{2 \alpha_{1}} \\
\epsilon_{2}-2 \alpha_{2} p_{2}+\alpha_{2} \lambda_{2}+\alpha_{2} \mu=0 \Rightarrow p_{2}=\frac{\epsilon_{2}+\alpha_{2} \lambda_{2}+\alpha_{2} \mu}{2 \alpha_{2}} \\
\lambda_{1}\left(\alpha_{1} p_{1}-\epsilon_{1}+K_{1}+K_{f}\right)=0 \\
\lambda_{2}\left(\alpha_{2} p_{2}-\epsilon_{2}+K_{2}+K_{f}\right)=0 \\
\mu\left(\alpha_{1} p_{1}+\alpha_{2} p_{2}-\epsilon_{1}-\epsilon_{2}+K_{1}+K_{2}+K_{f}\right)=0
\end{gathered}
$$

We define sets $E=\left\{\epsilon_{1} \leq 2 K_{1}+2 K_{f}, \quad \epsilon_{2} \leq 2 K_{2}+2 K_{f}, \quad \epsilon_{1}+\epsilon_{2} \leq 2 K_{1}+2 K_{2}+2 K_{f}\right\}$ and $F=\left\{2 \alpha_{1} K_{2}-2 \alpha_{2}\left(K_{1}+K_{f}\right) \leq \alpha_{1} \epsilon_{2}-\alpha_{2} \epsilon_{1} \leq-2 \alpha_{2} K_{1}+2 \alpha_{1}\left(K_{2}+K_{f}\right)\right\}$. Observe that the optimal solution to the unrestricted problem, $p_{i}^{(u)} \equiv \frac{\epsilon_{i}}{2 \alpha_{i}}, i=1,2$, is optimal for Problem $P_{2}^{\prime}$ (and $\left.\left(\mu, \lambda_{1}, \lambda_{2}\right)=0\right)$ if it satisfies all constraints of Problem $P_{2}^{\prime}$; that is, if 
$\left\{\epsilon_{1} \leq 2 K_{1}+2 K_{f}, \epsilon_{2} \leq 2 K_{2}+2 K_{f}, \epsilon_{1}+\epsilon_{2} \leq 2 K_{1}+2 K_{2}+2 K_{f}\right\}$. Otherwise (if $\left.E^{c}\right)$, the optimal solution will be on a boundary line of the feasible region (and its corresponding Lagrangean multiplier will be positive); see KKT conditions (23)-(25).

Consider case $E^{c}$. We first analyze the boundary solutions on line $\alpha_{1} p_{1}+\alpha_{2} p_{2}=$ $\epsilon_{1}+\epsilon_{2}-K_{1}-K_{2}-K_{f}\left(\mu \geq 0\right.$ and $\left.\lambda_{i}=0, i=1,2\right)$. Thus, setting $\lambda_{i}=0, i=1,2$, in $(21)$ and (22), we find the maximizing solution on this boundary line as:

$$
\begin{aligned}
p_{i} & =\frac{\epsilon_{i}}{2 \alpha_{i}}+\frac{\mu}{2}, i=1,2, \quad \text { where } \\
\mu & =\frac{\epsilon_{1}+\epsilon_{2}-2\left(K_{1}+K_{2}+K_{f}\right)}{\alpha_{1}+\alpha_{2}} .
\end{aligned}
$$

Substituting (27) in (26), we obtain:

$$
p_{i}=\frac{\epsilon_{i}}{2 \alpha_{i}}+\frac{\epsilon_{1}+\epsilon_{2}-2\left(K_{1}+K_{2}+K_{f}\right)}{2\left(\alpha_{1}+\alpha_{2}\right)}, \quad \text { for } i=1,2
$$

If this solution also satisfies constraints (7), then it will be optimal for Problem $P_{2}^{\prime}$. That is, if

$$
\begin{aligned}
\frac{\epsilon_{1}-K_{1}-K_{f}}{\alpha_{1}} & \leq \frac{\epsilon_{1}}{2 \alpha_{1}}+\frac{\epsilon_{1}+\epsilon_{2}-2\left(K_{1}+K_{2}+K_{f}\right)}{2\left(\alpha_{1}+\alpha_{2}\right)} \text { and } \\
\frac{\epsilon_{2}-K_{2}-K_{f}}{\alpha_{2}} & \leq \frac{\epsilon_{2}}{2 \alpha_{2}}+\frac{\epsilon_{1}+\epsilon_{2}-2\left(K_{1}+K_{2}+K_{f}\right)}{2\left(\alpha_{1}+\alpha_{2}\right)} \\
\Leftrightarrow 2 \alpha_{1} K_{2}-2 \alpha_{2}\left(K_{1}+K_{f}\right) & \leq \alpha_{1} \epsilon_{2}-\alpha_{2} \epsilon_{1} \leq-2 \alpha_{2} K_{1}+2 \alpha_{1}\left(K_{2}+K_{f}\right),
\end{aligned}
$$

or equivalently, if $E^{c} F$, then it is the optimal solution. If not (if $E^{c} F^{c}$ ), then the optimal solution must be on one of the boundary lines $p_{i}=\frac{\epsilon_{i}-K_{i}-K_{f}}{\alpha_{i}}, i=1,2$, of the feasible 
region. Consider the boundary line $p_{1}=\frac{\epsilon_{1}-K_{1}-K_{f}}{\alpha_{1}}$ (i.e., $\left.\lambda_{1} \geq 0, \lambda_{2}=0, \mu=0\right)$, which yields a demand for class 1 of $d_{1}=K_{1}+K_{f}$. Thus, Problem $P_{2}^{\prime}$ reduces to:

$$
\begin{aligned}
& \max _{p_{2}} \quad p_{2}\left(\epsilon_{2}-\alpha_{2} p_{2}\right) \\
& \text { subject to } \\
& p_{2} \geq \frac{\epsilon_{2}-K_{2}}{\alpha_{2}} \\
& p_{2} \geq 0
\end{aligned}
$$

Since the objective function is concave in $p_{2}$, the maximizing solution on this boundary line is given by

$$
p_{1}=\frac{\epsilon_{1}-K_{1}-K_{f}}{\alpha_{1}}, \quad p_{2}=\max \left(\frac{\epsilon_{2}}{2 \alpha_{2}}, \frac{\epsilon_{2}-K_{2}}{\alpha_{2}}\right) .
$$

Similarly, on boundary line $p_{2}=\frac{\epsilon_{2}-K_{2}-K_{f}}{\alpha_{2}}, p_{1}=\max \left(\frac{\epsilon_{1}}{2 \alpha_{1}}, \frac{\epsilon_{1}-K_{1}}{\alpha_{1}}\right)$.

Thus, the optimal solution to Problem $P_{2}^{\prime}$ is as follows:

1. If $E$, then

$$
p_{i}=\frac{\epsilon_{i}}{2 \alpha_{i}}, \quad \text { for } i=1,2
$$

2. If $E^{c} F$, then

$$
p_{i}=\frac{\epsilon_{i}}{2 \alpha_{i}}+\frac{\epsilon_{1}+\epsilon_{2}-2\left(K_{1}+K_{2}+K_{f}\right)}{2\left(\alpha_{1}+\alpha_{2}\right)}, \quad \text { for } i=1,2 .
$$

3. Else (if $E^{c} F^{c}$ ), the optimal solution is the best of the following solutions: 
(a) $p_{1}=\frac{\epsilon_{1}-K_{1}-K_{f}}{\alpha_{1}}, \quad p_{2}=\max \left(\frac{\epsilon_{2}}{2 \alpha_{2}}, \frac{\epsilon_{2}-K_{2}}{\alpha_{2}}\right)$.

(b) $p_{2}=\frac{\epsilon_{2}-K_{2}-K_{f}}{\alpha_{2}}, \quad p_{1}=\max \left(\frac{\epsilon_{1}}{2 \alpha_{1}}, \frac{\epsilon_{1}-K_{1}}{\alpha_{1}}\right)$.

We analyze the optimal solution in region $E^{c} F^{c}$ by decomposing this region into four disjoint sets: (i) $\left\{E^{c} F^{c}, \epsilon_{1}<2 K_{1}, \epsilon_{2}<2 K_{2}\right\}$, (ii) $\left\{E^{c} F^{c}, \epsilon_{1}>2 K_{1}, \epsilon_{2}<2 K_{2}\right\}$, (iii) $\left\{E^{c} F^{c}, \epsilon_{1}>2 K_{1}, \epsilon_{2}>2 K_{2}\right\}$, (iv) $\left\{E^{c} F^{c}, \epsilon_{1}<2 K_{1}, \epsilon_{2}>2 K_{2}\right\}$.

(i) Observe that $\left\{E^{c} F^{c}, \epsilon_{1}<2 K_{1}, \epsilon_{2}<2 K_{2}\right\}=\emptyset$.

(ii) If $\left\{E^{c} F^{c}, \epsilon_{1}>2 K_{1}, \epsilon_{2}<2 K_{2}\right\}$, then the optimal solution is the best of

$$
\vec{p}=\left(\frac{\epsilon_{1}-K_{1}-K_{f}}{\alpha_{1}}, \frac{\epsilon_{2}}{2 \alpha_{2}}\right) \quad \text { and } \quad \vec{p}=\left(\frac{\epsilon_{1}-K_{1}}{\alpha_{1}}, \frac{\epsilon_{2}-K_{2}-K_{f}}{\alpha_{2}}\right) .
$$

(iii) If $\left\{E^{c} F^{c}, \epsilon_{1}>2 K_{1}, \epsilon_{2}>2 K_{2}\right\}$, then the optimal solution is the best of

$$
\vec{p}=\left(\frac{\epsilon_{1}-K_{1}-K_{f}}{\alpha_{1}}, \frac{\epsilon_{2}-K_{2}}{\alpha_{2}}\right) \text { and } \vec{p}=\left(\frac{\epsilon_{1}-K_{1}}{\alpha_{1}}, \frac{\epsilon_{2}-K_{2}-K_{f}}{\alpha_{2}}\right) .
$$

(iv) If $\left\{E^{c} F^{c}, \epsilon_{1}<2 K_{1}, \epsilon_{2}>2 K_{2}\right\}$, then the optimal solution is the best of

$$
\vec{p}=\left(\frac{\epsilon_{1}-K_{1}-K_{f}}{\alpha_{1}}, \frac{\epsilon_{2}-K_{2}}{\alpha_{2}}\right) \quad \text { and } \quad \vec{p}=\left(\frac{\epsilon_{1}}{2 \alpha_{1}}, \frac{\epsilon_{2}-K_{2}-K_{f}}{\alpha_{2}}\right)
$$

Consider again case (ii), where $\left\{E^{c} F^{c}, \epsilon_{1}>2 K_{1}, \epsilon_{2}<2 K_{2}\right\}$. In this case, the optimal solution is given by $\vec{p}=\left(\frac{\epsilon_{1}-K_{1}-K_{f}}{\alpha_{1}}, \frac{\epsilon_{2}}{2 \alpha_{2}}\right)$ if

$$
\frac{\left(\epsilon_{1}-K_{1}-K_{f}\right)\left(K_{1}+K_{f}\right)}{\alpha_{1}}+\frac{\epsilon_{2}^{2}}{4 \alpha_{2}}>\frac{\left(\epsilon_{1}-K_{1}\right) K_{1}}{\alpha_{1}}+\frac{\left(\epsilon_{2}-K_{2}-K_{f}\right)\left(K_{2}+K_{f}\right)}{\alpha_{2}}
$$


$\Rightarrow 4 \alpha_{1} \epsilon_{2}\left(K_{2}+K_{f}\right)-\alpha_{1} \epsilon_{2}^{2}-4 \alpha_{2} \epsilon_{1} K_{f}<4 \alpha_{1}\left(K_{2}+K_{f}\right)^{2}-4 \alpha_{2} K_{f}\left(2 K_{1}+K_{f}\right) \Rightarrow K_{f}\left(\alpha_{1} \epsilon_{2}-\right.$ $\left.\alpha_{2} \epsilon_{1}\right)<\alpha_{1}\left(K_{2}+K_{f}\right)^{2}-\alpha_{2} K_{f}\left(2 K_{1}+K_{f}\right)+\frac{1}{4} \alpha_{1} \epsilon_{2}^{2}-\alpha_{1} \epsilon_{2} K_{2} \equiv G$.

Hence, if $\left\{E^{c} F^{c}, \epsilon_{1}>2 K_{1}, \epsilon_{2}<2 K_{2}, G\right\}$, then the optimal solution is $\vec{p}=\left(\frac{\epsilon_{1}-K_{1}-K_{f}}{\alpha_{1}}, \frac{\epsilon_{2}}{2 \alpha_{2}}\right)$. Observe that

$$
\left\{E^{c} F^{c}, \epsilon_{1}>2 K_{1}, \epsilon_{2}<2 K_{2}\right\}=\left\{\epsilon_{1}>2 K_{1}+2 K_{f}, \epsilon_{2}<2 K_{2}\right\}
$$

which implies that:

$$
\begin{aligned}
K_{f}\left(\alpha_{1} \epsilon_{2}-\alpha_{2} \epsilon_{1}\right) & <\alpha_{1} K_{f}\left(2 K_{2}\right)-\alpha_{2} K_{f}\left(2 K_{1}+2 K_{f}\right) \quad\left(\text { since } \epsilon_{1}>2 K_{1}+2 K_{f} \text { and } \epsilon_{2}<2 K_{2}\right) \\
& <\alpha_{1}\left(K_{2}+K_{f}\right)^{2}-\alpha_{2} K_{f}\left(2 K_{1}+K_{f}\right)+\frac{1}{4} \alpha_{1} \epsilon_{2}^{2}-\alpha_{1} \epsilon_{2} K_{2} .
\end{aligned}
$$

Thus,

$$
\left\{E^{c} F^{c}, \epsilon_{1}>2 K_{1}, \epsilon_{2}<2 K_{2}\right\} \Rightarrow\left\{E^{c} F^{c}, \epsilon_{1}>2 K_{1}, \epsilon_{2}<2 K_{2}, G\right\}
$$

Consequently, if $\left\{E^{c} F^{c}, \epsilon_{1}>2 K_{1}, \epsilon_{2}<2 K_{2}\right\}=\left\{\epsilon_{1}>2 K_{1}+2 K_{f}, \epsilon_{2}<2 K_{2}\right\}$, then the optimal solution is $\vec{p}=\left(\frac{\epsilon_{1}-K_{1}-K_{f}}{\alpha_{1}}, \frac{\epsilon_{2}}{2 \alpha_{2}}\right)$.

Cases (iii) and (iv) are analyzed in a similar way, obtaining the desired results. This completes the proof. 


\section{B Proof of Lemma 5.1}

In what follows, we prove that $V(\vec{K})$ is strictly concave in the investment vector $\vec{K}=$ $\left(K_{1}, K_{2}, K_{f}\right)$. For this purpose, we first derive the Hessian matrix of $V(\vec{K})$ corresponding to $\vec{K}$ (this can be derived either using the Leibnizs' rule or using the definition of the derivative).

We define the following non-negative elements:

$$
\begin{aligned}
a & \equiv \frac{2}{\left(\alpha_{1}+\alpha_{2}\right)} \iint_{\Omega_{2}} f_{1}\left(\epsilon_{1}\right) f_{2}\left(\epsilon_{2}\right) d \epsilon_{1} d \epsilon_{2} \\
b & \equiv \frac{2}{\alpha_{1}} \iint_{\Omega_{3}} f_{1}\left(\epsilon_{1}\right) f_{2}\left(\epsilon_{2}\right) d \epsilon_{1} d \epsilon_{2}+\frac{2}{\alpha_{1}} \iint_{\Omega_{6}} f_{1}\left(\epsilon_{1}\right) f_{2}\left(\epsilon_{2}\right) d \epsilon_{1} d \epsilon_{2} \\
c & \equiv \frac{2}{\alpha_{1}} \iint_{\Omega_{4}} f_{1}\left(\epsilon_{1}\right) f_{2}\left(\epsilon_{2}\right) d \epsilon_{2} d \epsilon_{1} \\
d & \equiv \frac{2}{\alpha_{2}} \iint_{\Omega_{3}} f_{1}\left(\epsilon_{1}\right) f_{2}\left(\epsilon_{2}\right) d \epsilon_{1} d \epsilon_{2} \\
e & \equiv \frac{2}{\alpha_{2}} \iint_{\Omega_{4}} f_{1}\left(\epsilon_{1}\right) f_{2}\left(\epsilon_{2}\right) d \epsilon_{2} d \epsilon_{1}+\frac{2}{\alpha_{2}} \iint_{\Omega_{5}} f_{1}\left(\epsilon_{1}\right) f_{2}\left(\epsilon_{2}\right) d \epsilon_{1} d \epsilon_{2}
\end{aligned}
$$

Then, we can write $-\mathbf{H}$, negative of the Hessian matrix of $\vec{K}$ corresponding to $\vec{K}$, as follows:

$$
-\mathbf{H}=\left(\begin{array}{ccc}
a+b+c & a & a+b \\
a & a+d+e & a+e \\
a+b & a+e & a+b+e
\end{array}\right)
$$

Next we apply the super diagonalization algorithm to check the positive definiteness of -H (see, for instance, Bazaraa, Sherali, and Shetty (1993)).

Observing that all elements on the diagonal are non-negative, $\mathbf{- H}$ reduces to following 
form by elementary row operations:

$$
-\mathbf{H}=\left(\begin{array}{ccc}
a+b+c & a & a+b \\
0 & a+d+e-\frac{a^{2}}{a+b+c} & a+e-\frac{a(a+b)}{a+b+c} \\
0 & a+e-\frac{a(a+b)}{a+b+c} & a+b+e-\frac{(a+b)^{2}}{a+b+c}
\end{array}\right)
$$

Let

$$
\mathbf{G}_{\text {new }} \equiv\left(\begin{array}{cc}
a+d+e-\frac{a^{2}}{a+b+c} & a+e-\frac{a(a+b)}{a+b+c} \\
a+e-\frac{a(a+b)}{a+b+c} & a+b+e-\frac{(a+b)^{2}}{a+b+c}
\end{array}\right)=\left(\begin{array}{cc}
g_{11} & g_{12} \\
g_{21} & g_{22}
\end{array}\right)
$$

where $g_{12}=g_{21}$.

Observe that for any continuous distribution of $\xi_{1}$ and $\xi_{2}$ defined over $[0, \infty)$, we have:

$$
\begin{aligned}
g_{11} & =\frac{a(b+c+d+e)+(b+c)(d+e)}{a+b+c}>0 \\
g_{22} & =\frac{(a+b)(c+e)+c e}{a+b+c}>0 \\
\Rightarrow g_{11} g_{22}-g_{12}^{2} & =\frac{a b c+a b e+a c d+a d e+b c d+b c e+b d e+c d e}{a+b+c}>0
\end{aligned}
$$

Eq.s (30), (31), and (32) imply that $\mathbf{G}_{\text {new }}$ is positive definite, and therefore, $\mathbf{-} \mathbf{H}$ is positive definite. Thus $\mathbf{H}$ is negative definite, and therefore, we conclude that $E[\Pi]$ is jointly concave in $\vec{K}$. Since $V(\vec{K})=E[\Pi]-\sum_{i=1,2, f} c_{i} K_{i}$, we conclude that $V(\vec{K})$ is strictly concave in $\vec{K}$. This completes the proof. 


\section{Proof of Lemma 5.3}

Consider the boundary solution $\vec{K}^{F}=\left(K_{1}^{F}=0, K_{2}^{F}=0, K_{f}^{F}>0\right)$, which corresponds to optimal Lagrangean multipliers of $v_{1} \geq 0, v_{2} \geq 0, v_{f}=0$. At solution $\vec{K}^{F}$, our state space reduces to:

$$
\begin{aligned}
& \Omega_{1}^{F}=\left\{\xi_{1}+\xi_{2}<2 K_{f}^{F}\right\} \\
& \Omega_{2}^{F}=\left\{\xi_{1}+\xi_{2}>2 K_{f}^{F},-2 \alpha_{2} K_{f}^{F}<\alpha_{1} \xi_{2}-\alpha_{2} \xi_{1}<2 \alpha_{1} K_{f}^{F}\right\} \\
& \Omega_{3}^{F}=\left\{\alpha_{1} \xi_{2}-\alpha_{2} \xi_{1}<-2 \alpha_{2} K_{f}^{F}\right\} \\
& \Omega_{4}^{F}=\left\{\alpha_{1} \xi_{2}-\alpha_{2} \xi_{1}>2 \alpha_{1} K_{f}^{F}\right\} \\
& \Omega_{5}^{F}=\emptyset ; \Omega_{6}^{F}=\emptyset
\end{aligned}
$$

Thus, the first-order KKT necessary \& sufficient conditions, given in Theorem 5.1, reduce to:

$$
\begin{aligned}
& \text { KKT }-\mathbf{1}: \\
& E\left[\frac{\xi_{1}+\xi_{2}-2 K_{f}^{F}}{\alpha_{1}+\alpha_{2}} \mid \Omega_{2}^{F}\right] \operatorname{Pr}\left(\Omega_{2}^{F}\right)+E\left[\frac{\xi_{1}-2 K_{f}^{F}}{\alpha_{1}} \mid \Omega_{3}^{F}\right] \operatorname{Pr}\left(\Omega_{3}^{F}\right)+E\left[\frac{\xi_{1}}{\alpha_{1}} \mid \Omega_{4}^{F}\right] \operatorname{Pr}\left(\Omega_{4}^{F}\right) \\
& =c_{1}-v_{1}
\end{aligned}
$$

KKT -2 :

$$
\begin{aligned}
& E\left[\frac{\xi_{1}+\xi_{2}-2 K_{f}^{F}}{\alpha_{1}+\alpha_{2}} \mid \Omega_{2}^{F}\right] \operatorname{Pr}\left(\Omega_{2}^{F}\right)+E\left[\frac{\xi_{2}}{\alpha_{2}} \mid \Omega_{3}^{F}\right] \operatorname{Pr}\left(\Omega_{3}^{F}\right)+E\left[\frac{\xi_{2}-2 K_{f}^{F}}{\alpha_{2}} \mid \Omega_{4}^{F}\right] \operatorname{Pr}\left(\Omega_{4}^{F}\right) \\
& =c_{2}-v_{2}
\end{aligned}
$$

KKT -3 : 


$$
\begin{aligned}
& E\left[\frac{\xi_{1}+\xi_{2}-2 K_{f}^{F}}{\alpha_{1}+\alpha_{2}} \mid \Omega_{2}^{F}\right] \operatorname{Pr}\left(\Omega_{2}^{F}\right)+E\left[\frac{\xi_{1}-2 K_{f}^{F}}{\alpha_{1}} \mid \Omega_{3}^{F}\right] \operatorname{Pr}\left(\Omega_{3}^{F}\right)+E\left[\frac{\xi_{2}-2 K_{f}^{F}}{\alpha_{2}} \mid \Omega_{4}^{F}\right] \operatorname{Pr}\left(\Omega_{4}^{F}\right) \\
& =c_{f}
\end{aligned}
$$

Observe that when $\Omega_{3}^{F}=\emptyset$, KKT conditions (35) and (36) imply that $c_{f}=c_{2}-v_{2} \leq c_{2}$, which is a contradiction, since $c_{f}>c_{2}$ by definition. Similarly, when $\Omega_{4}^{F}=\emptyset$, by KKT conditions (34) and (36), $c_{f}=c_{1}-v_{1} \leq c_{1}$, which is a contradiction, since $c_{f}>c_{1}$ by definition. Thus, if $\left\{\left(\Omega_{3}^{F}=\emptyset\right)\right.$ or $\left.\left(\Omega_{4}^{F}=\emptyset\right)\right\}$, then $\vec{K}^{F}=\left(K_{1}^{F}=0, K_{2}^{F}=0, K_{f}^{F}>0\right)$ is not a possible solution.

Next, consider the boundary solution $\vec{K}^{1 F}=\left(K_{1}^{1 F}>0, K_{2}^{1 F}=0, K_{f}^{1 F}>0\right)$, which implies that $v_{1}=0, v_{2} \geq 0, v_{f}=0$. Then our state space reduces to the following:

$$
\begin{aligned}
& \Omega_{1}^{1 F}=\left\{\xi_{2}<2 K_{f}^{1 F}, \xi_{1}+\xi_{2}<2 K_{1}^{1 F}+2 K_{f}^{1 F}\right\} \\
& \Omega_{2}^{1 F}=\left\{\xi_{1}+\xi_{2}>2 K_{1}^{1 F}+2 K_{f}^{1 F},-2 \alpha_{2}\left(K_{1}^{1 F}+K_{f}^{1 F}\right)<\alpha_{1} \xi_{2}-\alpha_{2} \xi_{1}<-2 \alpha_{2} K_{1}^{1 F}+2 \alpha_{1} K_{f}^{1 F}\right\} \\
& \Omega_{3}^{1 F}=\left\{\alpha_{1} \xi_{2}-\alpha_{2} \xi_{1}<-2 \alpha_{2}\left(K_{1}^{1 F}+K_{f}^{1 F}\right)\right\} \\
& \Omega_{4}^{1 F}=\left\{\xi_{1}>2 K_{1}^{1 F}, \alpha_{1} \xi_{2}-\alpha_{2} \xi_{1}>-2 \alpha_{2} K_{1}^{1 F}+2 \alpha_{1} K_{f}^{1 F}\right\} \\
& \Omega_{5}^{1 F}=\left\{\xi_{1}<2 K_{1}^{1 F}, \xi_{2}>2 K_{f}^{1 F}\right\} ; \quad \Omega_{6}^{1 F}=\emptyset
\end{aligned}
$$

Thus, the first-order KKT necessary \& sufficient conditions reduce to the following:

$$
\begin{aligned}
& \text { KKT }-\mathbf{1}: \\
& E\left[\frac{\xi_{1}+\xi_{2}-2 K_{1}^{1 F}-2 K_{f}^{1 F}}{\alpha_{1}+\alpha_{2}} \mid \Omega_{2}^{1 F}\right] \operatorname{Pr}\left(\Omega_{2}^{1 F}\right)+E\left[\frac{\xi_{1}-2 K_{1}^{1 F}-2 K_{f}^{1 F}}{\alpha_{1}} \mid \Omega_{3}^{1 F}\right] \operatorname{Pr}\left(\Omega_{3}^{1 F}\right)
\end{aligned}
$$


$+E\left[\frac{\xi_{1}-2 K_{1}^{1 F}}{\alpha_{1}} \mid \Omega_{4}^{1 F}\right] \operatorname{Pr}\left(\Omega_{4}^{1 F}\right)=c_{1}$

KKT - 2 :

$E\left[\frac{\xi_{1}+\xi_{2}-2 K_{1}^{1 F}-2 K_{f}^{1 F}}{\alpha_{1}+\alpha_{2}} \mid \Omega_{2}^{1 F}\right] \operatorname{Pr}\left(\Omega_{2}^{1 F}\right)+E\left[\frac{\xi_{2}}{\alpha_{2}} \mid \Omega_{3}^{1 F}\right] \operatorname{Pr}\left(\Omega_{3}^{1 F}\right)$
$+E\left[\frac{\xi_{2}-2 K_{f}^{1 F}}{\alpha_{2}} \mid \Omega_{4}^{1 F}\right] \operatorname{Pr}\left(\Omega_{4}^{1 F}\right)+E\left[\frac{\xi_{2}-2 K_{f}^{1 F}}{\alpha_{2}} \mid \Omega_{5}^{1 F}\right] \operatorname{Pr}\left(\Omega_{5}^{1 F}\right)=c_{2}-v_{2}$

KKT - 3 :

$$
\begin{aligned}
& E\left[\frac{\xi_{1}+\xi_{2}-2 K_{1}^{1 F}-2 K_{f}^{1 F}}{\alpha_{1}+\alpha_{2}} \mid \Omega_{2}^{1 F}\right] \operatorname{Pr}\left(\Omega_{2}^{1 F}\right)+E\left[\frac{\xi_{1}-2 K_{1}^{1 F}-2 K_{f}^{1 F}}{\alpha_{1}} \mid \Omega_{3}^{1 F}\right] \operatorname{Pr}\left(\Omega_{3}^{1 F}\right) \\
& +E\left[\frac{\xi_{2}-2 K_{f}^{1 F}}{\alpha_{2}} \mid \Omega_{4}^{1 F}\right] \operatorname{Pr}\left(\Omega_{4}^{1 F}\right)+E\left[\frac{\xi_{2}-2 K_{f}^{1 F}}{\alpha_{2}} \mid \Omega_{5}^{1 F}\right] \operatorname{Pr}\left(\Omega_{5}^{1 F}\right)=c_{f}
\end{aligned}
$$

Observe that when $\Omega_{3}^{1 F}=\emptyset$, KKT conditions (39) and (40) imply that $c_{f}=c_{2}-v_{2} \leq c_{2}$, which is a contradiction, since $c_{f}>c_{2}$ by definition. Similarly, when $\left(\Omega_{4}^{1 F}=\emptyset, \Omega_{5}^{1 F}=\emptyset\right)$, by KKT conditions (38) and (40), $c_{f}=c_{1}$, which is a contradiction, since $c_{f}>c_{1}$ by definition. So, if $\left\{\Omega_{3}^{1 F}=\emptyset\right.$ or $\left.\left(\Omega_{4}^{1 F}=\emptyset, \Omega_{5}^{1 F}=\emptyset\right)\right\}$, then $\vec{K}^{1 F}=\left(K_{1}^{1 F}>0, K_{2}^{1 F}=0, K_{f}^{1 F}>0\right)$ is not a possible solution.

At boundary solution $\vec{K}^{2 F}=\left(K_{1}^{2 F}=0, K_{2}^{2 F}>0, K_{f}^{2 F}>0\right)$, which implies that $v_{1} \geq 0, v_{2}=0, v_{f}=0$, our state space reduces to the following:

$$
\begin{aligned}
& \Omega_{1}^{2 F}=\left\{\xi_{1}<2 K_{f}^{2 F}, \xi_{1}+\xi_{2}<2 K_{2}^{2 F}+2 K_{f}^{2 F}\right\} \\
& \Omega_{2}^{2 F}=\left\{\xi_{1}+\xi_{2}>2 K_{2}^{2 F}+2 K_{f}^{2 F}, 2 \alpha_{1} K_{2}^{2 F}-2 \alpha_{2} K_{f}^{2 F}<\alpha_{1} \xi_{2}-\alpha_{2} \xi_{1}<2 \alpha_{1}\left(K_{2}^{2 F}+K_{f}^{2 F}\right)\right\} \\
& \Omega_{3}^{2 F}=\left\{\xi_{2}>2 K_{2}^{2 F}, \alpha_{1} \xi_{2}-\alpha_{2} \xi_{1}<2 \alpha_{1} K_{2}^{2 F}-2 \alpha_{2} K_{f}^{2 F}\right\} \\
& \Omega_{4}^{2 F}=\left\{\alpha_{1} \xi_{2}-\alpha_{2} \xi_{1}>2 \alpha_{1}\left(K_{2}^{2 F}+K_{f}^{2 F}\right)\right\} \\
& \Omega_{5}^{2 F}=\emptyset ; \quad \Omega_{6}^{2 F}=\left\{\xi_{1}>2 K_{f}^{2 F}, \xi_{2}<2 K_{2}^{2 F}\right\}
\end{aligned}
$$


Thus, the first-order KKT necessary \& sufficient conditions reduce to the following:

\section{KKT -1 :}

$$
\begin{aligned}
& E\left[\frac{\xi_{1}+\xi_{2}-2 K_{2}^{2 F}-2 K_{f}^{2 F}}{\alpha_{1}+\alpha_{2}} \mid \Omega_{2}^{2 F}\right] \operatorname{Pr}\left(\Omega_{2}^{2 F}\right)+E\left[\frac{\xi_{1}-2 K_{f}^{2 F}}{\alpha_{1}} \mid \Omega_{3}^{2 F}\right] \operatorname{Pr}\left(\Omega_{3}^{2 F}\right) \\
& +E\left[\frac{\xi_{1}}{\alpha_{1}} \mid \Omega_{4}^{2 F}\right] \operatorname{Pr}\left(\Omega_{4}^{2 F}\right)+E\left[\frac{\xi_{1}-2 K_{f}^{2 F}}{\alpha_{1}} \mid \Omega_{6}^{2 F}\right] \operatorname{Pr}\left(\Omega_{6}^{2 F}\right)=c_{1}-v_{1}
\end{aligned}
$$

\section{KKT - 2 :}

$$
\begin{aligned}
& E\left[\frac{\xi_{1}+\xi_{2}-2 K_{2}^{2 F}-2 K_{f}^{2 F}}{\alpha_{1}+\alpha_{2}} \mid \Omega_{2}^{2 F}\right] \operatorname{Pr}\left(\Omega_{2}^{2 F}\right)+E\left[\frac{\xi_{2}-2 K_{2}^{2 F}}{\alpha_{2}} \mid \Omega_{3}^{2 F}\right] \operatorname{Pr}\left(\Omega_{3}^{2 F}\right) \\
& +E\left[\frac{\xi_{2}-2 K_{2}^{2 F}-2 K_{f}^{2 F}}{\alpha_{2}} \mid \Omega_{4}^{2 F}\right] \operatorname{Pr}\left(\Omega_{4}^{2 F}\right)=c_{2}
\end{aligned}
$$

\section{KKT - 3 :}

$$
\begin{aligned}
& E\left[\frac{\xi_{1}+\xi_{2}-2 K_{2}^{2 F}-2 K_{f}^{2 F}}{\alpha_{1}+\alpha_{2}} \mid \Omega_{2}^{2 F}\right] \operatorname{Pr}\left(\Omega_{2}^{2 F}\right)+E\left[\frac{\xi_{1}-2 K_{f}^{2 F}}{\alpha_{1}} \mid \Omega_{3}^{2 F}\right] \operatorname{Pr}\left(\Omega_{3}^{2 F}\right) \\
& +E\left[\frac{\xi_{2}-2 K_{2}^{2 F}-2 K_{f}^{2 F}}{\alpha_{2}} \mid \Omega_{4}^{2 F}\right] \operatorname{Pr}\left(\Omega_{4}^{2 F}\right)+E\left[\frac{\xi_{1}-2 K_{f}^{2 F}}{\alpha_{1}} \mid \Omega_{6}^{2 F}\right] \operatorname{Pr}\left(\Omega_{6}^{2 F}\right)=c_{f}
\end{aligned}
$$

Observe that when $\Omega_{4}^{2 F}=\emptyset$, KKT conditions (42) and (44) imply that $c_{f}=c_{1}-v_{1} \leq c_{1}$, which is a contradiction, since $c_{f}>c_{1}$ by definition. Similarly, when $\left(\Omega_{3}^{2 F}=\emptyset, \Omega_{6}^{2 F}=\emptyset\right)$, by KKT conditions (43) and (44), $c_{f}=c_{2}$, which is a contradiction, since $c_{f}>c_{2}$ by definition. Thus, if $\left\{\Omega_{4}^{2 F}=\emptyset\right.$ or $\left.\left(\Omega_{3}^{2 F}=\emptyset, \Omega_{6}^{2 F}=\emptyset\right)\right\}$, then $\vec{K}^{2 F}=\left(K_{1}^{2 F}=0, K_{2}^{2 F}>0, K_{f}^{2 F}>0\right)$ is not a possible solution.

At boundary solution $\vec{K}^{A}=\left(K_{1}>0, K_{2}^{A}>0, K_{f}^{A}>0\right)$, the firm invests in all resources, which implies that $v_{1}=0, v_{2}=0, v_{f}=0$. Thus, the state space is the same as the general case, given in Eq. (14), where $\Omega_{i}^{A}=\Omega_{i}, i=1, \ldots, 6$. 
Thus, the first-order KKT necessary \& sufficient conditions are given in the following:

$$
\begin{aligned}
& \mathbf{K K T}-\mathbf{1}: \\
& E\left[\frac{\xi_{1}+\xi_{2}-2 K_{1}^{A}-2 K_{2}^{A}-2 K_{f}^{A}}{\alpha_{1}+\alpha_{2}} \mid \Omega_{2}^{A}\right] \operatorname{Pr}\left(\Omega_{2}^{A}\right)+E\left[\frac{\xi_{1}-2 K_{1}^{A}-2 K_{f}^{A}}{\alpha_{1}} \mid \Omega_{3}^{A}\right] \operatorname{Pr}\left(\Omega_{3}^{A}\right) \\
& +E\left[\frac{\xi_{1}-2 K_{1}^{A}}{\alpha_{1}} \mid \Omega_{4}^{A}\right] \operatorname{Pr}\left(\Omega_{4}^{A}\right)+E\left[\frac{\xi_{1}-2 K_{1}^{A}-2 K_{f}^{A}}{\alpha_{1}} \mid \Omega_{6}^{A}\right] \operatorname{Pr}\left(\Omega_{6}^{A}\right)=c_{1} \\
& \mathbf{K K T}-\mathbf{2}: \\
& E\left[\frac{\xi_{1}+\xi_{2}-2 K_{1}^{A}-2 K_{2}^{A}-2 K_{f}^{A}}{\alpha_{1}+\alpha_{2}} \mid \Omega_{2}^{A}\right] \operatorname{Pr}\left(\Omega_{2}^{A}\right)+E\left[\frac{\xi_{2}-2 K_{2}^{A}}{\alpha_{2}} \mid \Omega_{3}^{A}\right] \operatorname{Pr}\left(\Omega_{3}^{A}\right) \\
& +E\left[\frac{\xi_{2}-2 K_{2}^{A}-2 K_{f}^{A}}{\alpha_{2}} \mid \Omega_{4}^{A}\right] \operatorname{Pr}\left(\Omega_{4}^{A}\right)+E\left[\frac{\xi_{2}-2 K_{2}^{A}-2 K_{f}^{A}}{\alpha_{2}} \mid \Omega_{5}^{A}\right] \operatorname{Pr}\left(\Omega_{5}^{A}\right)=c_{2}
\end{aligned}
$$

KKT -3 :

$$
\begin{aligned}
& E\left[\frac{\xi_{1}+\xi_{2}-2 K_{1}^{A}-2 K_{2}^{A}-2 K_{f}^{A}}{\alpha_{1}+\alpha_{2}} \mid \Omega_{2}^{A}\right] \operatorname{Pr}\left(\Omega_{2}^{A}\right)+E\left[\frac{\xi_{1}-2 K_{1}^{A}-2 K_{f}^{A}}{\alpha_{1}} \mid \Omega_{3}^{A}\right] \operatorname{Pr}\left(\Omega_{3}^{A}\right) \\
& +E\left[\frac{\xi_{2}-2 K_{2}^{A}-2 K_{f}^{A}}{\alpha_{2}} \mid \Omega_{4}^{A}\right] \operatorname{Pr}\left(\Omega_{4}^{A}\right)+E\left[\frac{\xi_{2}-2 K_{2}^{A}-2 K_{f}^{A}}{\alpha_{2}} \mid \Omega_{5}^{A}\right] \operatorname{Pr}\left(\Omega_{5}^{A}\right) \\
& +E\left[\frac{\xi_{1}-2 K_{1}^{A}-2 K_{f}^{A}}{\alpha_{1}} \mid \Omega_{6}^{A}\right] \operatorname{Pr}\left(\Omega_{6}^{A}\right)=c_{f}
\end{aligned}
$$

Observe that when $\left(\Omega_{4}^{A}=\emptyset, \Omega_{5}^{A}=\emptyset\right)$, KKT conditions in Eq.s (45) and (47) imply that $c_{f}=c_{1}$, which is a contradiction, since $c_{f}>c_{1}$ by definition. Similarly, when $\left(\Omega_{3}^{A}=\right.$ $\left.\emptyset, \Omega_{6}^{A}=\emptyset\right)$, KKT conditions (46) and (47) imply that $c_{f}=c_{2}$, which is a contradiction, since $c_{f}>c_{2}$ by definition. Thus if $\left\{\left(\Omega_{4}^{A}=\emptyset, \Omega_{5}^{A}=\emptyset\right)\right.$ or $\left.\left(\Omega_{3}^{A}=\emptyset, \Omega_{6}^{A}=\emptyset\right)\right\}$, then $\vec{K}^{A}=\left(K_{1}^{A}>0, K_{2}^{A}>0, K_{f}^{A}>0\right)$ is not a possible solution. This completes the proof.

\section{Proof of Lemma 6.2}

Recall that by Lemma $5.2, K_{f}^{*}>0$ in the optimal solution only if $c_{f}<\underline{c}_{f}$. Thus, in the following, we analyze whether or not this is possible in each case. 


\section{D.1 Case 1: $\frac{\alpha_{1} c_{1}}{a}>\alpha_{2} c_{2}$}

Since $\frac{\alpha_{1} c_{1}}{a}>\alpha_{2} c_{2}$, it follows by Lemma 6.1 that $\frac{K_{1}^{D}}{a}<K_{2}^{D}$.

\section{D.1.1 Subcase 1: $\alpha_{1}>a \alpha_{2}$}

In this case, at boundary solution $\vec{K}^{D}$ our state space, given in Eq. (15), reduces to the following:

$$
\begin{aligned}
& \Omega_{1}^{D}=\left\{\xi<\frac{2 K_{1}^{D}}{a}, \xi<2 K_{2}^{D}\right\}=\left\{\xi<\frac{2 K_{1}^{D}}{a}\right\} \\
& \Omega_{2}^{D}=\emptyset \\
& \Omega_{3}^{D}=\left\{\xi>2 K_{2}^{D}, \xi\left(\alpha_{1}-a \alpha_{2}\right)<2 \alpha_{1} K_{2}^{D}-2 \alpha_{2} K_{1}^{D}\right\}=\left\{2 K_{2}^{D}<\xi<\frac{2 \alpha_{1} K_{2}^{D}-2 \alpha_{2} K_{1}^{D}}{\alpha_{1}-a \alpha_{2}}\right\} \\
& \Omega_{4}^{D}=\left\{\xi>\frac{2 K_{1}^{D}}{a}, \xi\left(\alpha_{1}-a \alpha_{2}\right)>2 \alpha_{1} K_{2}^{D}-2 \alpha_{2} K_{1}^{D}\right\}=\left\{\xi>\frac{-2 \alpha_{2} K_{1}^{D}+2 \alpha_{1} K_{2}^{D}}{\alpha_{1}-a \alpha_{2}}\right\} \\
& \Omega_{5}^{D}=\left\{\xi<\frac{2 K_{1}^{D}}{a}, \xi>2 K_{2}^{D}\right\}=\emptyset \\
& \Omega_{6}^{D}=\left\{\xi>\frac{2 K_{1}^{D}}{a}, \xi<2 K_{2}^{D}\right\}=\left\{\frac{2 K_{1}^{D}}{a}<\xi<2 K_{2}^{D}\right\}
\end{aligned}
$$

Thus, the first-order KKT necessary \& sufficient conditions for boundary solution $\vec{K}^{D}$, given in Eq.s (16) and (18), reduce to the following:

KKT $-1:$

$$
\begin{aligned}
& E\left[\frac{a \xi-2 K_{1}^{D}}{\alpha_{1}} \mid \Omega_{3}^{D}\right] \operatorname{Pr}\left(\Omega_{3}^{D}\right)+E\left[\frac{a \xi-2 K_{1}^{D}}{\alpha_{1}} \mid \Omega_{4}^{D}\right] \operatorname{Pr}\left(\Omega_{4}^{D}\right)+E\left[\frac{a \xi-2 K_{1}^{D}}{\alpha_{1}} \mid \Omega_{6}^{D}\right] \operatorname{Pr}\left(\Omega_{6}^{D}\right) \\
& =c_{1}
\end{aligned}
$$

KKT - 2 :

$$
E\left[\frac{\xi-2 K_{2}^{D}}{\alpha_{2}} \mid \Omega_{3}^{D}\right] \operatorname{Pr}\left(\Omega_{3}^{D}\right)+E\left[\frac{\xi-2 K_{2}^{D}}{\alpha_{2}} \mid \Omega_{4}^{D}\right] \operatorname{Pr}\left(\Omega_{4}^{D}\right)=c_{2}
$$


KKT -3 :

$$
\begin{aligned}
& E\left[\frac{a \xi-2 K_{1}^{D}}{\alpha_{1}} \mid \Omega_{3}^{D}\right] \operatorname{Pr}\left(\Omega_{3}^{D}\right)+E\left[\frac{\xi-2 K_{2}^{D}}{\alpha_{2}} \mid \Omega_{4}^{D}\right] \operatorname{Pr}\left(\Omega_{4}^{D}\right)+E\left[\frac{a \xi-2 K_{1}^{D}}{\alpha_{1}} \mid \Omega_{6}^{D}\right] \operatorname{Pr}\left(\Omega_{6}^{D}\right) \\
& =c_{f}-v_{f} \equiv \underline{c}_{f} \\
& \Rightarrow \underline{c}_{f}=c_{1}+c_{2}-\left\{\frac{a}{\alpha_{1}} E\left[\xi-\frac{2 K_{1}^{D}}{a} \mid \Omega_{4}^{D}\right] \operatorname{Pr}\left(\Omega_{4}^{D}\right)+\frac{1}{\alpha_{2}} E\left[\xi-2 K_{2}^{D} \mid \Omega_{3}^{D}\right] \operatorname{Pr}\left(\Omega_{3}^{D}\right)\right\}
\end{aligned}
$$

Recall that $K_{f}^{*}>0$ in the optimal solution if $c_{f}<\underline{c}_{f}$, as stated in Lemma 5.2. Also, by definition, $c_{1}, c_{2}<c_{f}<c_{1}+c_{2}$. Thus, in order to show that $K_{f}^{*}>0$ is possible in the optimal solution, we need to show that:

$$
\frac{a}{\alpha_{1}} E\left[\xi-\frac{2 K_{1}^{D}}{a} \mid \Omega_{4}^{D}\right] \operatorname{Pr}\left(\Omega_{4}^{D}\right)+\frac{1}{\alpha_{2}} E\left[\xi-2 K_{2}^{D} \mid \Omega_{3}^{D}\right] \operatorname{Pr}\left(\Omega_{3}^{D}\right)<\min \left(c_{1}, c_{2}\right) .
$$

(i) We first show that $\frac{a}{\alpha_{1}} E\left[\xi-\frac{2 K_{1}^{D}}{a} \mid \Omega_{4}^{D}\right] \operatorname{Pr}\left(\Omega_{4}^{D}\right)+\frac{1}{\alpha_{2}} E\left[\xi-2 K_{2}^{D} \mid \Omega_{3}^{D}\right] \operatorname{Pr}\left(\Omega_{3}^{D}\right)<c_{1}$. Observe that by Eq. (48), this is equivalent to showing that

$\underbrace{E\left[\xi\left(\alpha_{1}-a \alpha_{2}\right)-2 \alpha_{1} K_{2}^{D}+2 \alpha_{2} K_{1}^{D} \mid \Omega_{3}^{D}\right]}_{\left.<0 \text { (by definition of } \Omega_{3}^{\mathrm{D}}\right)} \operatorname{Pr}\left(\Omega_{3}^{D}\right)<a \alpha_{2} \underbrace{E\left[\xi-\frac{2 K_{1}^{D}}{a} \mid \Omega_{6}^{D}\right]}_{\left.>0 \text { (by definition of } \Omega_{6}^{D}\right)} \operatorname{Pr}\left(\Omega_{6}^{D}\right)$,

which always holds by definitions of $\Omega_{3}^{D}$ and $\Omega_{6}^{D}$, thus proving the first part.

(ii) Similarly, we next show that $\frac{a}{\alpha_{1}} E\left[\xi-\frac{2 K_{1}^{D}}{a} \mid \Omega_{4}^{D}\right] \operatorname{Pr}\left(\Omega_{4}^{D}\right)+\frac{1}{\alpha_{2}} E\left[\xi-2 K_{2}^{D} \mid \Omega_{3}^{D}\right] \operatorname{Pr}\left(\Omega_{3}^{D}\right)<$ $c_{2}$. Note that, by Eq. (49), this is equivalent to showing that

$$
E\left[\xi\left(\alpha_{1}-a \alpha_{2}\right)-2 \alpha_{1} K_{2}^{D}+2 \alpha_{2} K_{1}^{D} \mid \Omega_{4}^{D}\right] \operatorname{Pr}\left(\Omega_{4}^{D}\right)>0
$$

which always holds by definition of $\Omega_{4}^{D}$, thus proving the second part. 
Hence, $K_{f}^{*}>0$ is possible in the optimal solution in this case.

\section{D.1.2 Subcase 2: $\alpha_{1}=a \alpha_{2}$}

In this case, our state space reduces to the following:

$$
\begin{aligned}
& \Omega_{1}^{D}=\left\{\xi<\frac{2 K_{1}^{D}}{a}\right\} \\
& \Omega_{2}^{D}=\emptyset \\
& \Omega_{3}^{D}=\left\{\xi>2 K_{2}^{D}\right\} \\
& \Omega_{4}^{D}=\emptyset \\
& \Omega_{5}^{D}=\emptyset \\
& \Omega_{6}^{D}=\left\{\frac{2 K_{1}^{D}}{a}<\xi<2 K_{2}^{D}\right\}
\end{aligned}
$$

Thus, we have the following first-order KKT necessary \& sufficient conditions:

$$
\begin{aligned}
& \text { KKT - 1 : } E\left[\frac{a \xi-2 K_{1}^{D}}{\alpha_{1}} \mid \Omega_{3}^{D}\right] \operatorname{Pr}\left(\Omega_{3}^{D}\right)+E\left[\frac{a \xi-2 K_{1}^{D}}{\alpha_{1}} \mid \Omega_{6}^{D}\right] \operatorname{Pr}\left(\Omega_{6}^{D}\right)=c_{1} \\
& \mathbf{K K T}-\mathbf{2}: \quad E\left[\frac{\xi-2 K_{2}^{D}}{\alpha_{2}} \mid \Omega_{3}^{D}\right] \operatorname{Pr}\left(\Omega_{3}^{D}\right)=c_{2} \\
& \mathbf{K K T}-\mathbf{3}: E\left[\frac{a \xi-2 K_{1}^{D}}{\alpha_{1}} \mid \Omega_{3}^{D}\right] \operatorname{Pr}\left(\Omega_{3}^{D}\right)+E\left[\frac{a \xi-2 K_{1}^{D}}{\alpha_{1}} \mid \Omega_{6}^{D}\right] \operatorname{Pr}\left(\Omega_{6}^{D}\right)=c_{f}-v_{f}=\underline{c}_{f}(53)
\end{aligned}
$$

KKT conditions (51) and (53) imply that $\underline{c}_{f}=c_{1}$. By Lemma 5.2, $K_{f}^{*}>0$ in the optimal solution only if $c_{f}<\underline{c}_{f}=c_{1}$. However, by definition, $c_{f}>c_{1}=\underline{c}_{f}$. Thus, $K_{f}^{*}=0$ in the optimal solution. 


\section{D.1.3 Subcase 3: $\alpha_{1}<a \alpha_{2}$}

In this case, our state space reduces to the following:

$$
\begin{aligned}
& \Omega_{1}^{D}=\left\{\xi<\frac{2 K_{1}^{D}}{a}\right\} \\
& \Omega_{2}^{D}=\emptyset \\
& \Omega_{3}^{D}=\left\{\xi>2 K_{2}^{D}, \xi\left(\alpha_{1}-a \alpha_{2}\right)<-2 \alpha_{2} K_{1}^{D}+2 \alpha_{1} K_{2}^{D}\right\} \\
& \Omega_{4}^{D}=\left\{\xi>\frac{2 K_{1}^{D}}{a}, \xi\left(\alpha_{1}-a \alpha_{2}\right)>-2 \alpha_{2} K_{1}^{D}+2 \alpha_{1} K_{2}^{D}\right\} \\
& \Omega_{5}^{D}=\emptyset \\
& \Omega_{6}^{D}=\left\{\frac{2 K_{1}^{D}}{a}<\xi<2 K_{2}^{D}\right\}
\end{aligned}
$$

There are two cases to consider:

1. $2 \alpha_{1} K_{2}^{D}-2 \alpha_{2} K_{1}^{D} \geq 0 \Rightarrow\left\{\xi\left(\alpha_{1}-a \alpha_{2}\right) \leq-2 \alpha_{2} K_{1}^{D}+2 \alpha_{1} K_{2}^{D}\right\}=\Omega^{\cup}$, since $\alpha_{1}<a \alpha_{2}$ and $\xi>0$. Thus, $\Omega_{3}^{D}=\left\{\xi>2 K_{2}^{D}\right\}$ and $\Omega_{4}^{D}=\emptyset$.

2. $2 \alpha_{1} K_{2}^{D}-2 \alpha_{2} K_{1}^{D}<0 \Rightarrow \frac{2 \alpha_{2} K_{1}^{D}-2 \alpha_{1} K_{2}^{D}}{a \alpha_{2}-\alpha_{1}}<\frac{2 a \alpha_{2} \frac{K_{1}^{D}}{a}-2 \alpha_{1} \frac{K_{1}^{D}}{a}}{a \alpha_{2}-\alpha_{1}}=\frac{2 K_{1}^{D}}{a}$.

Thus, $\Omega_{3}^{D}=\left\{\xi>2 K_{2}^{D}\right\}$ and $\Omega_{4}^{D}=\emptyset$.

Thus, the first-order KKT necessary and sufficient conditions are the same as in subcase 2, which imply that $\underline{c}_{f}=c_{1}$. Therefore, $K_{f}^{*}=0$ in the optimal solution.

D.2 Case 2: $\frac{\alpha_{1} c_{1}}{a}=\alpha_{2} c_{2}$

Since $\frac{\alpha_{1} c_{1}}{a}=\alpha_{2} c_{2}$, it follows by Lemma 6.1 that $\frac{K_{1}^{D}}{a}=K_{2}^{D}$. 


\section{D.2.1 Subcase 1: $\alpha_{1}>a \alpha_{2}$}

In this case, our state space reduces to the following:

$$
\begin{aligned}
& \Omega_{1}^{D}=\left\{\xi<\frac{2 K_{1}^{D}}{a}, \xi<2 K_{2}^{D}\right\}=\left\{\xi<2 K_{2}^{D}\right\} \\
& \Omega_{2}^{D}=\emptyset \\
& \Omega_{3}^{D}=\left\{\xi>2 K_{2}^{D}, \xi<2 K_{2}^{D}\right\}=\emptyset \\
& \Omega_{4}^{D}=\left\{\xi>\frac{2 K_{1}^{D}}{a}\right\}=\left\{\xi>2 K_{2}^{D}\right\} \\
& \Omega_{5}^{D}=\left\{\xi<\frac{2 K_{1}^{D}}{a}, \xi>2 K_{2}^{D}\right\}=\emptyset \\
& \Omega_{6}^{D}=\left\{\xi>\frac{2 K_{1}^{D}}{a}, \xi<2 K_{2}^{D}\right\}=\emptyset
\end{aligned}
$$

Thus, we have the following first-order KKT necessary \& sufficient conditions:

$$
\begin{aligned}
& \mathbf{K K T}-\mathbf{1}: \quad E\left[\frac{a \xi-2 K_{1}^{D}}{\alpha_{1}} \mid \Omega_{4}^{D}\right] \operatorname{Pr}\left(\Omega_{4}^{D}\right)=c_{1} \\
& \mathbf{K K T}-\mathbf{2}: \quad E\left[\frac{\xi-2 K_{2}^{D}}{\alpha_{2}} \mid \Omega_{4}^{D}\right] \operatorname{Pr}\left(\Omega_{4}^{D}\right)=c_{2} \\
& \mathbf{K K T}-\mathbf{3}: \quad E\left[\frac{\xi-2 K_{2}^{D}}{\alpha_{2}} \mid \Omega_{4}^{D}\right] \operatorname{Pr}\left(\Omega_{4}^{D}\right)=c_{f}-v_{f}=\underline{c}_{f}
\end{aligned}
$$

KKT conditions (55) and (56) imply that $\underline{c}_{f}=c_{2}$. However, since by definition $c_{f}>$ $c_{2}=\underline{c}_{f}$, it follows, by Lemma 5.2 , that $K_{f}^{*}=0$ in the optimal solution.

\section{D.2.2 Subcase 2: $\alpha_{1}=a \alpha_{2}$}

In this case, our state space reduces to the following:

$$
\Omega_{1}^{D}=\left\{\xi<\frac{2 K_{1}^{D}}{a}\right\}=\left\{\xi<2 K_{2}^{D}\right\}
$$




$$
\begin{aligned}
& \Omega_{2}^{D}=\left\{\xi>2 K_{2}^{D}\right\} \\
& \Omega_{3}^{D}=\Omega_{4}^{D}=\Omega_{5}^{D}=\Omega_{6}^{D}=\emptyset
\end{aligned}
$$

Since $\Omega_{3}^{D}=\Omega_{4}^{D}=\Omega_{5}^{D}=\Omega_{6}^{D}=\emptyset$, the first-order KKT condition 3, given in Eq. (18), implies that $\underline{c}_{f}=0$. Thus, $K_{f}^{*}=0$ in the optimal solution by Lemma 5.2.

\section{D.2.3 Subcase 3: $\alpha_{1}<a \alpha_{2}$}

In this case, our state space reduces to the following:

$$
\begin{aligned}
& \Omega_{1}^{D}=\left\{\xi<\frac{2 K_{1}^{D}}{a}\right\}=\left\{\xi<2 K_{2}^{D}\right\} \\
& \Omega_{2}^{D}=\emptyset \\
& \Omega_{3}^{D}=\left\{\xi>2 K_{2}^{D}\right\} \\
& \Omega_{4}^{D}=\left\{\xi>\frac{2 K_{1}^{D}}{a}, \xi<2 K_{2}^{D}\right\}=\emptyset \\
& \Omega_{5}^{D}=\left\{\xi<\frac{2 K_{1}^{D}}{a}, \xi>2 K_{2}^{D}\right\}=\emptyset \\
& \Omega_{6}^{D}=\left\{\xi>\frac{2 K_{1}^{D}}{a}, \xi<2 K_{2}^{D}\right\}=\emptyset
\end{aligned}
$$

Thus, the first-order KKT necessary \& sufficient conditions, given in Eq.s (16) and (18), imply that $\underline{c}_{f}=c_{1}$. Since, by definition, $c_{f}>c_{1}=\underline{c}_{f}$, it follows, by Lemma 5.2 , that $K_{f}^{*}=0$ in the optimal solution.

\section{D.3 Case 3: $\frac{\alpha_{1} c_{1}}{a}<\alpha_{2} c_{2}$}

Since $\frac{\alpha_{1} c_{1}}{a}<\alpha_{2} c_{2}$, it follows by Lemma 6.1 that $\frac{K_{1}^{D}}{a}>K_{2}^{D}$. 


\section{D.3.1 Subcase 1: $\alpha_{1}>a \alpha_{2}$}

In this case, our state space reduces to the following:

$$
\begin{aligned}
& \Omega_{1}^{D}=\left\{\xi<2 K_{2}^{D}\right\} \\
& \Omega_{2}^{D}=\emptyset \\
& \Omega_{3}^{D}=\left\{\xi>2 K_{2}^{D}, \xi<\frac{2 \alpha_{1} K_{2}^{D}-2 \alpha_{2} K_{1}^{D}}{\alpha_{1}-a \alpha_{2}}\right\}=\emptyset \\
& \Omega_{4}^{D}=\left\{\xi>\frac{2 K_{1}^{D}}{a}, \xi>\frac{2 \alpha_{1} K_{2}^{D}-2 \alpha_{2} K_{1}^{D}}{\alpha_{1}-a \alpha_{2}}\right\}=\left\{\xi>\frac{2 K_{1}^{D}}{a}\right\} \\
& \Omega_{5}^{D}=\left\{2 K_{2}^{D}<\xi<\frac{2 K_{1}^{D}}{a}\right\} \\
& \Omega_{6}^{D}=\left\{\xi>\frac{2 K_{1}^{D}}{a}, \xi<2 K_{2}^{D}\right\}=\emptyset
\end{aligned}
$$

Thus, the first-order KKT necessary \& sufficient conditions reduce to the following:

$$
\begin{aligned}
& \text { KKT - 1 : } E\left[\frac{a \xi-2 K_{1}^{D}}{\alpha_{1}} \mid \Omega_{4}^{D}\right] \operatorname{Pr}\left(\Omega_{4}^{D}\right)=c_{1} \\
& \mathbf{K K T}-\mathbf{2}: \quad E\left[\frac{\xi-2 K_{2}^{D}}{\alpha_{2}} \mid \Omega_{4}^{D}\right] \operatorname{Pr}\left(\Omega_{4}^{D}\right)+E\left[\frac{\xi-2 K_{2}^{D}}{\alpha_{2}} \mid \Omega_{5}^{D}\right] \operatorname{Pr}\left(\Omega_{5}^{D}\right)=c_{2} \\
& \mathbf{K K T}-\mathbf{3}: \quad E\left[\frac{\xi-2 K_{2}^{D}}{\alpha_{2}} \mid \Omega_{4}^{D}\right] \operatorname{Pr}\left(\Omega_{4}^{D}\right)+E\left[\frac{\xi-2 K_{2}^{D}}{\alpha_{2}} \mid \Omega_{5}^{D}\right] \operatorname{Pr}\left(\Omega_{5}^{D}\right)=c_{f}-v_{f}=\underline{c}_{f}
\end{aligned}
$$

KKT conditions (58) and (59) imply that $\underline{c}_{f}=c_{2}$. However, since by definition $c_{f}>$ $c_{2}=\underline{c}_{f}$, Lemma 5.2 implies that $K_{f}^{*}=0$ in the optimal solution.

\section{D.3.2 Subcase 2: $\alpha_{1}=a \alpha_{2}$}

In this case, our state space reduces to the following:

$$
\Omega_{1}^{D}=\left\{\xi<2 K_{2}^{D}\right\}
$$




$$
\begin{aligned}
\Omega_{2}^{D} & =\emptyset \\
\Omega_{3}^{D} & =\left\{\xi>2 K_{2}^{D}, \xi\left(\alpha_{1}-a \alpha_{2}\right)<2 \alpha_{1} K_{2}^{D}-2 \alpha_{2} K_{1}^{D}\right\} \\
& =\{\xi>2 K_{2}^{D}, 0<\underbrace{2 \alpha_{1} K_{2}^{D}-2 \alpha_{2} K_{1}^{D}}_{<0\left(\text { since } K_{2}^{D}<\frac{K_{1}^{D}}{a}\right)}\}=\emptyset \\
\Omega_{4}^{D} & =\left\{\xi>\frac{2 K_{1}^{D}}{a}, \xi\left(\alpha_{1}-a \alpha_{2}\right)>2 \alpha_{1} K_{2}^{D}-2 \alpha_{2} K_{1}^{D}\right\} \\
& =\left\{\xi>\frac{2 K_{1}^{D}}{a}\right\} \\
\Omega_{5}^{D} & =\left\{2 K_{2}^{D}<\xi<\frac{2 K_{1}^{D}}{a}\right\} \\
\Omega_{6}^{D} & =\left\{\xi>\frac{2 K_{1}^{D}}{a}, \xi<2 K_{2}^{D}\right\}=\emptyset
\end{aligned}
$$

Thus, the first-order KKT necessary \& sufficient conditions are the same as in subcase 1. Therefore, $K_{f}^{*}=0$ in the optimal solution.

\section{D.3.3 Subcase 3: $\alpha_{1}<a \alpha_{2}$}

In this case, our state space reduces to the following:

$$
\begin{aligned}
& \Omega_{1}^{D}=\left\{\xi<2 K_{2}^{D}\right\} \\
& \Omega_{2}^{D}=\emptyset \\
& \Omega_{3}^{D}=\left\{\xi>2 K_{2}^{D}, \xi>\frac{2 \alpha_{2} K_{1}^{D}-2 \alpha_{1} K_{2}^{D}}{a \alpha_{2}-\alpha_{1}}\right\}=\left\{\xi>\frac{2 \alpha_{2} K_{1}^{D}-2 \alpha_{1} K_{2}^{D}}{a \alpha_{2}-\alpha_{1}}\right\} \\
& \Omega_{4}^{D}=\left\{\xi>\frac{2 K_{1}^{D}}{a}, \xi<\frac{2 \alpha_{2} K_{1}^{D}-2 \alpha_{1} K_{2}^{D}}{a \alpha_{2}-\alpha_{1}}\right\}=\left\{\frac{2 K_{1}^{D}}{a}<\xi<\frac{2 \alpha_{2} K_{1}^{D}-2 \alpha_{1} K_{2}^{D}}{a \alpha_{2}-\alpha_{1}}\right\} \\
& \Omega_{5}^{D}=\left\{2 K_{2}^{D}<\xi<\frac{2 K_{1}^{D}}{a}\right\} \\
& \Omega_{6}^{D}=\left\{\frac{2 K_{1}^{D}}{a}<\xi<2 K_{2}^{D}\right\}=\emptyset
\end{aligned}
$$


Thus, we have the following first-order KKT necessary \& sufficient conditions:

$$
\begin{aligned}
& \text { KKT }-\mathbf{1}: \\
& E\left[\frac{a \xi-2 K_{1}^{D}}{\alpha_{1}} \mid \Omega_{3}^{D}\right] \operatorname{Pr}\left(\Omega_{3}^{D}\right)+E\left[\frac{a \xi-2 K_{1}^{D}}{\alpha_{1}} \mid \Omega_{4}^{D}\right] \operatorname{Pr}\left(\Omega_{4}^{D}\right)=c_{1}
\end{aligned}
$$

\section{KKT - 2 :}

$E\left[\frac{\xi-2 K_{2}^{D}}{\alpha_{2}} \mid \Omega_{3}^{D}\right] \operatorname{Pr}\left(\Omega_{3}^{D}\right)+E\left[\frac{\xi-2 K_{2}^{D}}{\alpha_{2}} \mid \Omega_{4}^{D}\right] \operatorname{Pr}\left(\Omega_{4}^{D}\right)+E\left[\frac{\xi-2 K_{2}^{D}}{\alpha_{2}} \mid \Omega_{5}^{D}\right] \operatorname{Pr}\left(\Omega_{5}^{D}\right)=c_{2}(61)$

KKT -3 :

$$
\begin{aligned}
& E\left[\frac{a \xi-2 K_{1}^{D}}{\alpha_{1}} \mid \Omega_{3}^{D}\right] \operatorname{Pr}\left(\Omega_{3}^{D}\right)+E\left[\frac{\xi-2 K_{2}^{D}}{\alpha_{2}} \mid \Omega_{4}^{D}\right] \operatorname{Pr}\left(\Omega_{4}^{D}\right)+E\left[\frac{\xi-2 K_{2}^{D}}{\alpha_{2}} \mid \Omega_{5}^{D}\right] \operatorname{Pr}\left(\Omega_{5}^{D}\right) \\
& =c_{f}-v_{f}=\underline{c}_{f} \\
& \Rightarrow \underline{c}_{f}=c_{1}+c_{2}-\left\{\frac{a}{\alpha_{1}} E\left[\xi-\frac{2 K_{1}^{D}}{a} \mid \Omega_{4}^{D}\right] \operatorname{Pr}\left(\Omega_{4}^{D}\right)+\frac{1}{\alpha_{2}} E\left[\xi-2 K_{2}^{D} \mid \Omega_{3}^{D}\right] \operatorname{Pr}\left(\Omega_{3}^{D}\right)\right\}
\end{aligned}
$$

Recall that $K_{f}^{*}>0$ in the optimal solution only if $c_{f}<\underline{c}_{f}$, as stated in Lemma 5.2. Also, by definition, $c_{1}, c_{2}<c_{f}<c_{1}+c_{2}$. Thus, in order to show that $K_{f}^{*}>0$ is possible in the optimal solution, we need to show that:

$$
\frac{a}{\alpha_{1}} E\left[\xi-\frac{2 K_{1}^{D}}{a} \mid \Omega_{4}^{D}\right] \operatorname{Pr}\left(\Omega_{4}^{D}\right)+\frac{1}{\alpha_{2}} E\left[\xi-2 K_{2}^{D} \mid \Omega_{3}^{D}\right] \operatorname{Pr}\left(\Omega_{3}^{D}\right)<\min \left(c_{1}, c_{2}\right)
$$

(i) We first show that $\frac{a}{\alpha_{1}} E\left[\xi-\frac{2 K_{1}^{D}}{a} \mid \Omega_{4}^{D}\right] \operatorname{Pr}\left(\Omega_{4}^{D}\right)+\frac{1}{\alpha_{2}} E\left[\xi-2 K_{2}^{D} \mid \Omega_{3}^{D}\right] \operatorname{Pr}\left(\Omega_{3}^{D}\right)<c_{1}$. Observe that, by Eq. (60), this is equivalent to showing that

$$
\left(a \alpha_{2}-\alpha_{1}\right) \underbrace{E\left[\xi-\frac{\left(2 \alpha_{2} K_{1}^{D}-2 \alpha_{1} K_{2}^{D}\right)}{a \alpha_{2}-\alpha_{1}} \mid \Omega_{3}^{D}\right]}_{\left.>0 \text { (by definition of } \Omega_{3}^{D}\right)} \operatorname{Pr}\left(\Omega_{3}^{D}\right)>0,
$$

which always holds by definition of $\Omega_{3}^{D}$, thus proving the first part. 
(ii) We next show that $\frac{a}{\alpha_{1}} E\left[\xi-\frac{2 K_{1}^{D}}{a} \mid \Omega_{4}^{D}\right] \operatorname{Pr}\left(\Omega_{4}^{D}\right)+\frac{1}{\alpha_{2}} E\left[\xi-2 K_{2}^{D} \mid \Omega_{3}^{D}\right] \operatorname{Pr}\left(\Omega_{3}^{D}\right)<c_{2}$. Observe that, by Eq. (61), this is equivalent to showing that

$$
\left(a \alpha_{2}-\alpha_{1}\right) \underbrace{E\left[\xi-\frac{\left(2 \alpha_{2} K_{1}^{D}-2 \alpha_{1} K_{2}^{D}\right)}{a \alpha_{2}-\alpha_{1}} \mid \Omega_{4}^{D}\right]}_{\left.<0 \text { (by definition of } \Omega_{4}^{D}\right)} \operatorname{Pr}\left(\Omega_{4}^{D}\right)<\alpha_{1} \underbrace{E\left[\xi-2 K_{2}^{D} \mid \Omega_{5}^{D}\right]}_{>0 \text { (by definition of } \Omega_{5}^{D} \text { ) }} \operatorname{Pr}\left(\Omega_{5}^{D}\right),
$$

which always holds by definitions of $\Omega_{4}^{D}$ and $\Omega_{5}^{D}$, and since $a \alpha_{2}>\alpha_{1}$ by assumption, thus proving the second part. Hence $K_{f}^{*}>0$ is possible in the optimal solution in this case. This completes the proof.

\section{E Proof of Theorem 6.1 parts (2a) and (2b)}

\section{E.1 Proof of (2a)}

In the following, we first prove that $\frac{K_{f}^{2 F}}{a}<K_{2}^{2 F}$, and then, using this result, we prove the other inequalities.

\section{E.1.1 Proof of $\frac{K_{f}^{2 F}}{a}<K_{2}^{2 F}$}

Suppose that $\vec{K}^{2 F}$ is the optimal solution. Suppose, to the contrary, that $\frac{K_{f}^{2 F}}{a} \geq K_{2}^{2 F}$. In addition, recall that $\alpha_{1}>a \alpha_{2}$. Then, we can show that the following inequalities hold:

$$
\begin{gathered}
\frac{2 \alpha_{1} K_{2}^{2 F}-2 \alpha_{2} K_{f}^{2 F}}{\alpha_{1}-a \alpha_{2}} \leq 2 K_{2}^{2 F} \leq \frac{2 K_{2}^{2 F}+2 K_{f}^{2 F}}{1+a} \leq \frac{2 K_{f}^{2 F}}{a} \\
\text { and } \frac{2 \alpha_{1}\left(K_{2}^{2 F}+K_{f}^{2 F}\right)}{\alpha_{1}-a \alpha_{2}} \geq \frac{2 K_{2}^{2 F}+2 K_{f}^{2 F}}{1+a}
\end{gathered}
$$


Thus, if $\frac{K_{f}^{2 F}}{a} \geq K_{2}^{2 F}$, then $\Omega_{3}^{2 F}=\left\{\xi>2 K_{2}^{2 F}, \xi<\frac{2 \alpha_{1} K_{2}^{2 F}-2 \alpha_{2} K_{f}^{2 F}}{\alpha_{1}-a \alpha_{2}}\right\}=\emptyset$ and $\Omega_{6}^{2 F}=\{\xi>$ $\left.\frac{2 K_{f}^{2 F}}{a}, \xi<2 K_{2}^{2 F}\right\}=\emptyset$, which follow by Eq. (41). Hence, by Lemma $5.3, \vec{K}^{2 F}$ is not a possible solution, which is a contradiction. Thus, we must have $\frac{K_{f}^{2 F}}{a}<K_{2}^{2 F}$. This implies the following inequalities:

$$
\frac{2 K_{f}^{2 F}}{a}<\frac{2 K_{2}^{2 F}+2 K_{f}^{2 F}}{1+a}<2 K_{2}^{2 F}<\frac{2 \alpha_{1} K_{2}^{2 F}-2 \alpha_{2} K_{f}^{2 F}}{\alpha_{1}-a \alpha_{2}}
$$

Thus, our state space reduces to the one depicted in Figure 2.

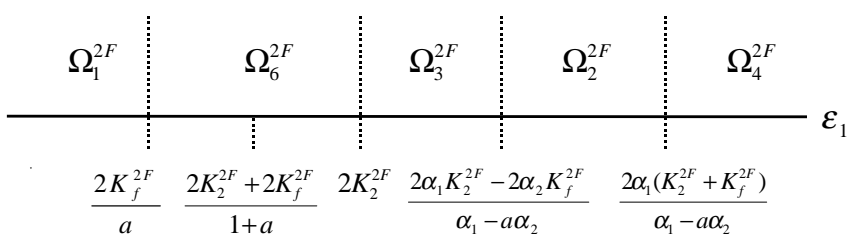

Figure 2: The demand space at solution $\vec{K}^{2 F}$ for perfectly positively correlated demand patterns. 


\section{E.1.2 $\underline{\text { Proof of } K_{2}^{2 F}<K_{2}^{I}}$}

Observe that we can write:

$$
\begin{aligned}
& E\left[\frac{\xi(1+a)-2 K_{2}^{2 F}-2 K_{f}^{2 F}}{\alpha_{1}+\alpha_{2}} \mid \Omega_{2}^{2 F}\right] \\
& =E\left[\frac{\xi-2 K_{2}^{2 F}}{\alpha_{2}} \mid \Omega_{2}^{2 F}\right]+E\left[\frac{2 \alpha_{1} K_{2}^{2 F}-2 \alpha_{2} K_{f}^{2 F}-\xi\left(\alpha_{1}-a \alpha_{2}\right)}{\alpha_{2}\left(\alpha_{1}+\alpha_{2}\right)} \mid \Omega_{2}^{2 F}\right]
\end{aligned}
$$

Using Eq. (63), KKT condition, given in Eq. (43), can be written as:

$$
\begin{aligned}
& E\left[\xi-2 K_{2}^{2 F} \mid \Omega_{2}^{2 F}\right] \operatorname{Pr}\left(\Omega_{2}^{2 F}\right)+E\left[\frac{2 \alpha_{1} K_{2}^{2 F}-2 \alpha_{2} K_{f}^{2 F}-\xi\left(\alpha_{1}-a \alpha_{2}\right)}{\alpha_{1}+\alpha_{2}} \mid \Omega_{2}^{2 F}\right] \operatorname{Pr}\left(\Omega_{2}^{2 F}\right) \\
& +E\left[\xi-2 K_{2}^{2 F} \mid \Omega_{3}^{2 F}\right] \operatorname{Pr}\left(\Omega_{3}^{2 F}\right)+E\left[\xi-2 K_{2}^{2 F} \mid \Omega_{4}^{2 F}\right] \operatorname{Pr}\left(\Omega_{4}^{2 F}\right)-2 K_{f}^{2 F} \operatorname{Pr}\left(\Omega_{4}^{2 F}\right)=\alpha_{2} c_{2}(64)
\end{aligned}
$$

Observe that $\left(\Omega_{2}^{2 F} \cup \Omega_{3}^{2 F} \cup \Omega_{4}^{2 F}\right)=\left\{\xi>2 K_{2}^{2 F}\right\}$ (see Figure 2).

Thus, we can write Eq. (64) as:

$$
\begin{aligned}
& E\left[\xi-2 K_{2}^{2 F} \mid \xi>2 K_{2}^{2 F}\right] \operatorname{Pr}\left(\xi>2 K_{2}^{2 F}\right) \\
& =\alpha_{2} c_{2}+2 K_{f}^{2 F} \operatorname{Pr}\left(\Omega_{4}^{2 F}\right)-\frac{1}{\alpha_{1}+\alpha_{2}} \underbrace{E\left[2 \alpha_{1} K_{2}^{2 F}-2 \alpha_{2} K_{f}^{2 F}-\xi\left(\alpha_{1}-a \alpha_{2}\right) \mid \Omega_{2}^{2 F}\right]}_{<0\left(\text { by definition of } \Omega_{2}^{2 F}\right)} \operatorname{Pr}\left(\Omega_{2}^{2 F}\right) \\
& >\alpha_{2} c_{2} .
\end{aligned}
$$

We now compare this optimality condition at solution $\vec{K}^{2 F}$ with the optimality condition at solution $\vec{K}^{I}$, given as follows (see Eq. (13)):

$$
E\left[\xi-2 K_{2}^{I} \mid \xi>2 K_{2}^{I}\right] \operatorname{Pr}\left(\xi>2 K_{2}^{I}\right)=\alpha_{2} c_{2}
$$


Hence,

$E\left[\xi-2 K_{2}^{2 F} \mid \xi>2 K_{2}^{2 F}\right] \operatorname{Pr}\left(\xi>2 K_{2}^{2 F}\right)>E\left[\xi-2 K_{2}^{I} \mid \xi>2 K_{2}^{I}\right] \operatorname{Pr}\left(\xi>2 K_{2}^{I}\right) \Rightarrow K_{2}^{2 F}<K_{2}^{I}$

\section{E.1.3 Proof of $K_{f}^{2 F}>K_{1}^{I}$}

Observe that:

$$
\begin{aligned}
& E\left[\frac{\xi(1+a)-2 K_{2}^{2 F}-2 K_{f}^{2 F}}{\alpha_{1}+\alpha_{2}} \mid \Omega_{2}^{2 F}\right] \operatorname{Pr}\left(\Omega_{2}^{2 F}\right) \\
& =E\left[\frac{a \xi-2 K_{f}^{2 F}}{\alpha_{1}} \mid \Omega_{2}^{2 F}\right] \operatorname{Pr}\left(\Omega_{2}^{2 F}\right)+E\left[\frac{2 \alpha_{2} K_{f}^{2 F}-2 \alpha_{1} K_{2}^{2 F}+\xi\left(\alpha_{1}-a \alpha_{2}\right)}{\alpha_{1}\left(\alpha_{1}+\alpha_{2}\right)} \mid \Omega_{2}^{2 F}\right] \operatorname{Pr}\left(\Omega_{2}^{2 F}\right)(65)
\end{aligned}
$$

Using Eq. (65), we can write the KKT condition in Eq. (42) as:

$$
\begin{aligned}
& \frac{a}{\alpha_{1}} E\left[\xi-\frac{2 K_{f}^{2 F}}{a} \mid \Omega_{2}^{2 F}\right] \operatorname{Pr}\left(\Omega_{2}^{2 F}\right)+E\left[\frac{2 \alpha_{2} K_{f}^{2 F}-2 \alpha_{1} K_{2}^{2 F}+\xi\left(\alpha_{1}-a \alpha_{2}\right)}{\alpha_{1}\left(\alpha_{1}+\alpha_{2}\right)} \mid \Omega_{2}^{2 F}\right] \operatorname{Pr}\left(\Omega_{2}^{2 F}\right) \\
& +\frac{a}{\alpha_{1}} E\left[\xi-\frac{2 K_{f}^{2 F}}{a} \mid \Omega_{3}^{2 F}\right] \operatorname{Pr}\left(\Omega_{3}^{2 F}\right)+\frac{a}{\alpha_{1}} E\left[\xi-\frac{2 K_{f}^{2 F}}{a} \mid \Omega_{4}^{2 F}\right] \operatorname{Pr}\left(\Omega_{4}^{2 F}\right)+\frac{2 K_{f}^{2 F}}{\alpha_{1}} \operatorname{Pr}\left(\Omega_{4}^{2 F}\right) \\
& +\frac{a}{\alpha_{1}} E\left[\xi-\frac{2 K_{f}^{2 F}}{a} \mid \Omega_{6}^{2 F}\right] \operatorname{Pr}\left(\Omega_{6}^{2 F}\right)=c_{1}-v_{1}
\end{aligned}
$$

Observe that $\left(\Omega_{2}^{2 F} \cup \Omega_{3}^{2 F} \cup \Omega_{4}^{2 F} \cup \Omega_{6}^{2 F}\right)=\left\{\xi>\frac{2 K_{f}^{2 F}}{a}\right\}$ (see Figure 2). Thus, Eq. (66) can be written as:

$$
\begin{aligned}
& E\left[\xi-\frac{2 K_{f}^{2 F}}{a} \mid \xi>\frac{2 K_{f}^{2 F}}{a}\right] \operatorname{Pr}\left(\xi>\frac{2 K_{f}^{2 F}}{a}\right) \\
& =\frac{\alpha_{1}}{a}\left(c_{1}-v_{1}\right)-\frac{2 K_{f}^{2 F}}{a} \operatorname{Pr}\left(\Omega_{4}^{2 F}\right) \\
& -\frac{1}{a\left(\alpha_{1}+\alpha_{2}\right)} \underbrace{E\left[2 \alpha_{2} K_{f}^{2 F}-2 \alpha_{1} K_{2}^{2 F}+\xi\left(\alpha_{1}-a \alpha_{2}\right) \mid \Omega_{2}^{2 F}\right]}_{\left.>0 \text { (by definition of } \Omega_{2}^{2 F}\right)} \operatorname{Pr}\left(\Omega_{2}^{2 F}\right)
\end{aligned}
$$




$$
<\frac{\alpha_{1} c_{1}}{a}
$$

Next, we compare this optimality condition at solution $\vec{K}^{2 F}$ with the optimality condition at solution $\vec{K}^{I}$, given as follows (see Eq. (13)):

$$
E\left[\xi-\frac{2 K_{1}^{I}}{a} \mid \xi>\frac{2 K_{1}^{I}}{a}\right] \operatorname{Pr}\left(\xi>\frac{2 K_{1}^{I}}{a}\right)=\frac{\alpha_{1} c_{1}}{a} .
$$

Thus, $E\left[\xi-\frac{2 K_{f}^{2 F}}{a} \mid \xi>\frac{2 K_{f}^{2 F}}{a}\right] \operatorname{Pr}\left(\xi>\frac{2 K_{f}^{2 F}}{a}\right)<E\left[\xi-\frac{2 K_{1}^{I}}{a} \mid \xi>\frac{2 K_{1}^{I}}{a}\right] \operatorname{Pr}\left(\xi>\frac{2 K_{1}^{I}}{a}\right)$, which implies that $K_{f}^{2 F}>K_{1}^{I}$.

Recall that we have already shown that $\frac{K_{f}^{2 F}}{a}<K_{2}^{2 F}$ and $K_{2}^{2 F}<K_{2}^{I}$. Thus,

$$
\frac{K_{1}^{I}}{a}<\frac{K_{f}^{2 F}}{a}<K_{2}^{2 F}<K_{2}^{I}
$$

which completes the proof of part 2(a) of Theorem 6.1 .

\section{E.2 Proof of (2b)}

At $\vec{K}^{A}=\left\{K_{1}^{A}>0, K_{2}^{A}>0, K_{f}^{A}>0\right\}$, our state space is given by the following:

$$
\begin{aligned}
& \Omega_{1}=\left\{\xi<\frac{2 K_{1}^{A}+2 K_{f}^{A}}{a}, \xi<2 K_{2}^{A}+2 K_{f}^{A}, \xi<\frac{2 K_{1}^{A}+2 K_{2}^{A}+2 K_{f}^{A}}{1+a}\right\} \\
& \Omega_{2}=\left\{\frac{2 \alpha_{1} K_{2}^{A}-2 \alpha_{2}\left(K_{1}^{A}+K_{f}^{A}\right)}{\alpha_{1}-a \alpha_{2}}<\xi<\frac{-2 \alpha_{2} K_{1}^{A}+2 \alpha_{1}\left(K_{2}^{A}+K_{f}^{A}\right)}{\alpha_{1}-a \alpha_{2}}, \xi>\frac{2 K_{1}^{A}+2 K_{2}^{A}+2 K_{f}^{A}}{1+a}\right\} \\
& \Omega_{3}=\left\{\xi>2 K_{2}^{A}, \xi<\frac{2 \alpha_{1} K_{2}^{A}-2 \alpha_{2}\left(K_{1}^{A}+K_{f}^{A}\right)}{\alpha_{1}-a \alpha_{2}}\right\} \\
& \Omega_{4}=\left\{\xi>\frac{2 K_{1}^{A}}{a}, \xi>\frac{-2 \alpha_{2} K_{1}^{A}+2 \alpha_{1}\left(K_{2}^{A}+K_{f}^{A}\right)}{\alpha_{1}-a \alpha_{2}}\right\}
\end{aligned}
$$




$$
\begin{aligned}
& \Omega_{5}=\left\{\xi<\frac{2 K_{1}^{A}}{a}, \xi>2 K_{2}^{A}+2 K_{f}^{A}\right\} \\
& \Omega_{6}=\left\{\xi>\frac{2 K_{1}^{A}+2 K_{f}^{A}}{a}, \xi<2 K_{2}^{A}\right\}
\end{aligned}
$$

Thus, the first-order KKT necessary \& sufficient conditions are given in the following:

$$
\begin{aligned}
& \text { KKT - 1 : } \\
& E\left[\frac{\xi(1+a)-2 K_{1}^{A}-2 K_{2}^{A}-2 K_{f}^{A}}{\alpha_{1}+\alpha_{2}} \mid \Omega_{2}^{A}\right] \operatorname{Pr}\left(\Omega_{2}^{A}\right)+a E\left[\frac{\xi-\frac{2 K_{1}^{A}}{a}-\frac{2 K_{f}^{A}}{a}}{\alpha_{1}} \mid \Omega_{3}^{A}\right] \operatorname{Pr}\left(\Omega_{3}^{A}\right) \\
& +a E\left[\frac{\xi-\frac{2 K_{1}^{A}}{a}}{\alpha_{1}} \mid \Omega_{4}^{A}\right] \operatorname{Pr}\left(\Omega_{4}^{A}\right)+a E\left[\frac{\xi-\frac{2 K_{1}^{A}}{a}-\frac{2 K_{f}^{A}}{a}}{\alpha_{1}} \mid \Omega_{6}^{A}\right] \operatorname{Pr}\left(\Omega_{6}^{A}\right)=c_{1}
\end{aligned}
$$

KKT - 2 :

$$
\begin{aligned}
& E\left[\frac{\xi(1+a)-2 K_{1}^{A}-2 K_{2}^{A}-2 K_{f}^{A}}{\alpha_{1}+\alpha_{2}} \mid \Omega_{2}^{A}\right] \operatorname{Pr}\left(\Omega_{2}^{A}\right)+E\left[\frac{\xi-2 K_{2}^{A}}{\alpha_{2}} \mid \Omega_{3}^{A}\right] \operatorname{Pr}\left(\Omega_{3}^{A}\right) \\
& +E\left[\frac{\xi-2 K_{2}^{A}-2 K_{f}^{A}}{\alpha_{2}} \mid \Omega_{4}^{A}\right] \operatorname{Pr}\left(\Omega_{4}^{A}\right)+E\left[\frac{\xi-2 K_{2}^{A}-2 K_{f}^{A}}{\alpha_{2}} \mid \Omega_{5}^{A}\right] \operatorname{Pr}\left(\Omega_{5}^{A}\right)=c_{2}
\end{aligned}
$$

\section{KKT -3 :}

$$
\begin{aligned}
& E\left[\frac{\xi(1+a)-2 K_{1}^{A}-2 K_{2}^{A}-2 K_{f}^{A}}{\alpha_{1}+\alpha_{2}} \mid \Omega_{2}^{A}\right] \operatorname{Pr}\left(\Omega_{2}^{A}\right)+a E\left[\frac{\xi-\frac{2 K_{1}^{A}}{a}-\frac{2 K_{f}^{A}}{a}}{\alpha_{1}} \mid \Omega_{3}^{A}\right] \operatorname{Pr}\left(\Omega_{3}^{A}\right) \\
& +E\left[\frac{\xi-2 K_{2}^{A}-2 K_{f}^{A}}{\alpha_{2}} \mid \Omega_{4}^{A}\right] \operatorname{Pr}\left(\Omega_{4}^{A}\right)+E\left[\frac{\xi-2 K_{2}^{A}-2 K_{f}^{A}}{\alpha_{2}} \mid \Omega_{5}^{A}\right] \operatorname{Pr}\left(\Omega_{5}^{A}\right) \\
& +a E\left[\frac{\xi-\frac{2 K_{1}^{A}}{a}-\frac{2 K_{f}^{A}}{a}}{\alpha_{1}} \mid \Omega_{6}^{A}\right] \operatorname{Pr}\left(\Omega_{6}^{A}\right)=c_{f}
\end{aligned}
$$

In the following proof, we will make use of the KKT optimality conditions for solution $\vec{K}^{I}$, given in Eq. (13) in Lemma 3.1, which states that:

$$
\begin{aligned}
& E\left[\xi-\frac{2 K_{1}^{I}}{a} \mid \xi>\frac{2 K_{1}^{I}}{a}\right] \operatorname{Pr}\left(\xi>\frac{2 K_{1}^{I}}{a}\right)=\frac{\alpha_{1} c_{1}}{a} \\
& E\left[\xi-2 K_{2}^{I} \mid \xi>2 K_{2}^{I}\right] \operatorname{Pr}\left(\xi>2 K_{2}^{I}\right)=\alpha_{2} c_{2}
\end{aligned}
$$


In the following, we first show that $\frac{K_{1}^{A}+K_{f}^{A}}{a}<K_{2}^{A}$, and then use this result to prove the other inequalities.

The proof follows by contradiction. Suppose that $\vec{K}^{A}$ is the optimal solution. Suppose, to the contrary, that $\frac{K_{1}^{A}+K_{f}^{A}}{a} \geq K_{2}^{A}$. Then we have:

(1) $2 K_{2}^{A} \leq \frac{2 K_{1}^{A}+2 K_{2}^{A}+2 K_{f}^{A}}{1+a} \leq \frac{2 K_{1}^{A}+2 K_{f}^{A}}{a}$; and (2) $\frac{2 \alpha_{1} K_{2}^{A}-2 \alpha_{2}\left(K_{1}^{A}+K_{f}^{A}\right)}{\alpha_{1}-a \alpha_{2}} \leq 2 K_{2}^{A}$.

(1) and (2) imply that $\Omega_{3}^{A}=\emptyset$ and $\Omega_{6}^{A}=\emptyset$. However, Lemma 5.3 implies that in this case $\vec{K}^{A}$ is not a possible solution, which is a contradiction. Thus, if $\vec{K}^{A}$ is the optimal solution, then we must have $\frac{K_{1}^{A}+K_{f}^{A}}{a}<K_{2}^{A}$. In this case, it is easy to show that:

$$
\begin{gathered}
\frac{2 K_{1}^{A}+2 K_{f}^{A}}{a}<\frac{2 K_{1}^{A}+2 K_{2}^{A}+2 K_{f}^{A}}{1+a}<2 K_{2}^{A}<\frac{2 \alpha_{1} K_{2}^{A}-2 \alpha_{2}\left(K_{1}^{A}+K_{f}^{A}\right)}{\alpha_{1}-a \alpha_{2}} \\
<\frac{2 \alpha_{1} K_{2}^{A}+2 \alpha_{1} K_{f}^{A}-2 \alpha_{2} K_{1}^{A}}{\alpha_{1}-a \alpha_{2}}
\end{gathered}
$$

Thus, $\Omega_{5}^{A}=\emptyset$ and the state space reduces to the one given in Figure 3 .

In what follows, we analyze the relationships between $K_{1}^{A}, K_{2}^{A}, K_{f}^{A}$ and $K_{1}^{I}, K_{2}^{I}$.

\section{E.2.1 Proof of $K_{1}^{A}+K_{f}^{A}>K_{1}^{I}$}

Observe that:

$$
E\left[\frac{\xi(1+a)-2\left(K_{1}^{A}+K_{2}^{A}+K_{f}^{A}\right)}{\alpha_{1}+\alpha_{2}} \mid \Omega_{2}^{A}\right]
$$




\begin{tabular}{c:c:c:c:c}
$\Omega_{1}^{A}$ & $\Omega_{6}^{A}$ & $\Omega_{3}^{A}$ & $\Omega_{2}^{A}$ & $\Omega_{4}^{A}$ \\
\hdashline$\vdots$ & $\vdots K_{1}^{A}+2 K_{f}^{A}$ \\
$\frac{2 K_{1}^{A}+2 K_{+}^{A}+2 K_{f}^{A}}{1+a}$ & $K_{2}^{A}$ & $\frac{2 \alpha_{1} K_{2}^{A}-2 \alpha_{2}\left(K_{1}^{A}+K_{f}^{A}\right)}{\alpha_{1}-a \alpha_{2}} \frac{2 \alpha_{1} K_{2}^{A}+2 \alpha_{1} K_{f}^{A}-2 \alpha_{2} K_{1}^{A}}{\alpha_{1}-a \alpha_{2}}$
\end{tabular}

Figure 3: The demand space at solution $\vec{K}^{A}$ for perfectly positively correlated demand patterns.

$$
=E\left[\frac{a \xi-2\left(K_{1}^{A}+K_{f}^{A}\right)}{\alpha_{1}} \mid \Omega_{2}^{A}\right]+E\left[\frac{\xi\left(\alpha_{1}-a \alpha_{2}\right)-2 \alpha_{1} K_{2}^{A}+2 \alpha_{2}\left(K_{1}^{A}+K_{f}^{A}\right)}{\alpha_{1}\left(\alpha_{1}+\alpha_{2}\right)} \mid \Omega_{2}^{A}\right]
$$

Using Eq. (72) and observing that $\left(\Omega_{2}^{A} \cup \Omega_{3}^{A} \cup \Omega_{4}^{A} \cup \Omega_{6}^{A}\right)=\left\{\xi>\frac{2\left(K_{1}^{A}+K_{f}^{A}\right)}{a}\right\}$, we can write the KKT condition in Eq. (67) as follows:

$$
\begin{aligned}
& \frac{a}{\alpha_{1}} E\left[\xi-\frac{2\left(K_{1}^{A}+K_{f}^{A}\right)}{a} \mid \Omega_{2}^{A}\right] \operatorname{Pr}\left(\Omega_{2}^{A}\right)+E\left[\frac{\xi\left(\alpha_{1}-a \alpha_{2}\right)-2 \alpha_{1} K_{2}^{A}+2 \alpha_{2}\left(K_{1}^{A}+K_{f}^{A}\right)}{\alpha_{1}\left(\alpha_{1}+\alpha_{2}\right)} \mid \Omega_{2}^{A}\right] \operatorname{Pr}\left(\Omega_{2}^{A}\right) \\
& +\frac{a}{\alpha_{1}} E\left[\xi-\frac{2\left(K_{1}^{A}+K_{f}^{A}\right)}{a} \mid \Omega_{3}^{A}\right] \operatorname{Pr}\left(\Omega_{3}^{A}\right)+\frac{a}{\alpha_{1}} E\left[\xi-\frac{2\left(K_{1}^{A}+K_{f}^{A}\right)}{a} \mid \Omega_{4}^{A}\right] \operatorname{Pr}\left(\Omega_{4}^{A}\right) \\
& +\frac{a}{\alpha_{1}} \frac{2 K_{f}^{A}}{a} \operatorname{Pr}\left(\Omega_{4}^{A}\right)+\frac{a}{\alpha_{1}} E\left[\xi-\frac{2\left(K_{1}^{A}+K_{f}^{A}\right)}{a} \mid \Omega_{6}^{A}\right] \operatorname{Pr}\left(\Omega_{6}^{A}\right)=c_{1} \\
& \Rightarrow E\left[\xi-\frac{2\left(K_{1}^{A}+K_{f}^{A}\right)}{a} \mid \xi>\frac{2\left(K_{1}^{A}+K_{f}^{A}\right)}{a}\right] \operatorname{Pr}\left(\xi>\frac{2\left(K_{1}^{A}+K_{f}^{A}\right)}{a}\right) \\
& =\frac{\alpha_{1} c_{1}}{a}-\frac{2 K_{f}^{A}}{a} \operatorname{Pr}\left(\Omega_{4}^{A}\right)-\frac{\alpha_{1}}{a} E\left[\frac{\xi\left(\alpha_{1}-a \alpha_{2}\right)-2 \alpha_{1} K_{2}^{A}+2 \alpha_{2}\left(K_{1}^{A}+K_{f}^{A}\right)}{\alpha_{1}\left(\alpha_{1}+\alpha_{2}\right)} \mid \Omega_{2}^{A}\right] \operatorname{Pr}\left(\Omega_{2}^{A}\right)
\end{aligned}
$$




$$
\begin{aligned}
& =\frac{\alpha_{1} c_{1}}{a}-\frac{2 K_{f}^{A}}{a} \operatorname{Pr}\left(\Omega_{4}^{A}\right)-\frac{\left(\alpha_{1}-a \alpha_{2}\right)}{a\left(\alpha_{1}+\alpha_{2}\right)} \underbrace{E\left[\xi-\frac{\left(2 \alpha_{1} K_{2}^{A}-2 \alpha_{2}\left(K_{1}^{A}+K_{f}^{A}\right)\right)}{\alpha_{1}-a \alpha_{2}} \mid \Omega_{2}^{A}\right]}_{\left.>0 \text { (by definition of } \Omega_{2}^{A}\right)} \operatorname{Pr}\left(\Omega_{2}^{A}\right) \\
& <\frac{\alpha_{1} c_{1}}{a}
\end{aligned}
$$

Thus, Eq.s (70) and (73) imply that

$$
K_{1}^{A}+K_{f}^{A}>K_{1}^{D} .
$$

\section{E.2.2 Proof of $K_{1}^{A}<K_{1}^{I}$}

Observe that:

$$
\begin{aligned}
& E\left[\frac{\xi(1+a)-2 K_{1}^{A}-2 K_{2}^{A}-2 K_{f}^{A}}{\alpha_{1}+\alpha_{2}} \mid \Omega_{2}^{A}\right] \\
& =E\left[\frac{a \xi-2 K_{1}^{A}}{\alpha_{1}} \mid \Omega_{2}^{A}\right]+E\left[\frac{\xi\left(\alpha_{1}-a \alpha_{2}\right)+2 \alpha_{2} K_{1}^{A}-2 \alpha_{1} K_{2}^{A}-2 \alpha_{1} K_{f}^{A}}{\alpha_{1}\left(\alpha_{1}+\alpha_{2}\right)} \mid \Omega_{2}^{A}\right]
\end{aligned}
$$

Then we can write KKT condition in Eq. (67) as follows:

$$
\begin{aligned}
& \frac{a}{\alpha_{1}} E\left[\xi-\frac{2 K_{1}^{A}}{a} \mid \Omega_{2}^{A}\right] \operatorname{Pr}\left(\Omega_{2}^{A}\right)+E\left[\frac{\xi\left(\alpha_{1}-a \alpha_{2}\right)+2 \alpha_{2} K_{1}^{A}-2 \alpha_{1}\left(K_{2}^{A}+K_{f}^{A}\right)}{\alpha_{1}\left(\alpha_{1}+\alpha_{2}\right)} \mid \Omega_{2}^{A}\right] \operatorname{Pr}\left(\Omega_{2}^{A}\right) \\
& +\frac{a}{\alpha_{1}} E\left[\xi-\frac{2 K_{1}^{A}}{a} \mid \Omega_{3}^{A}\right] \operatorname{Pr}\left(\Omega_{3}^{A}\right)-\frac{a}{\alpha_{1}} \frac{2 K_{f}^{A}}{a} \operatorname{Pr}\left(\Omega_{3}^{A}\right)+\frac{a}{\alpha_{1}} E\left[\xi-\frac{2 K_{1}^{A}}{a} \mid \Omega_{4}^{A}\right] \operatorname{Pr}\left(\Omega_{4}^{A}\right) \\
& +\frac{a}{\alpha_{1}} E\left[\xi-\frac{2 K_{1}^{A}}{a} \mid \Omega_{6}^{A}\right] \operatorname{Pr}\left(\Omega_{6}^{A}\right)-\frac{a}{\alpha_{1}} \frac{2 K_{f}^{A}}{a} \operatorname{Pr}\left(\Omega_{6}^{A}\right)=c_{1} \\
& \Rightarrow E\left[\xi-\frac{2 K_{1}^{A}}{a} \mid \xi>\frac{2 K_{1}^{A}}{a}\right] \operatorname{Pr}\left(\xi>\frac{2 K_{1}^{A}}{a}\right) \\
& =\frac{\alpha_{1} c_{1}}{a}+\frac{2 K_{f}^{A}}{a} \operatorname{Pr}\left(\Omega_{3}^{A}\right)+\frac{2 K_{f}^{A}}{a} \operatorname{Pr}\left(\Omega_{6}^{A}\right) \\
& +E\left[\xi-\frac{2 K_{1}^{A}}{a} \mid \frac{2 K_{1}^{A}}{a}<\xi<\frac{2 K_{1}^{A}+2 K_{f}^{A}}{a}\right] \operatorname{Pr}\left(\frac{2 K_{1}^{A}}{a}<\xi<\frac{2 K_{1}^{A}+2 K_{f}^{A}}{a}\right)
\end{aligned}
$$




$$
\begin{aligned}
& -\underbrace{E\left[\frac{\xi\left(\alpha_{1}-a \alpha_{2}\right)+2 \alpha_{2} K_{1}^{A}-2 \alpha_{1} K_{2}^{A}-2 \alpha_{1} K_{f}^{A}}{a\left(\alpha_{1}+\alpha_{2}\right)} \mid \Omega_{2}^{A}\right]}_{\left.<0 \text { (by definition of } \Omega_{2}^{A}\right)} \operatorname{Pr}\left(\Omega_{2}^{A}\right) \\
& >\frac{\alpha_{1} c_{1}}{a} \quad
\end{aligned}
$$

Thus, Eq.s (70) and (77) imply that

$$
K_{1}^{A}<K_{1}^{I}
$$

\section{E.2.3 Proof of $K_{2}^{A}<K_{2}^{I}$}

Observe that:

$$
\begin{aligned}
& E\left[\frac{\xi(1+a)-2 K_{1}^{A}-2 K_{2}^{A}-2 K_{f}^{A}}{\alpha_{1}+\alpha_{2}}\right] \\
& =E\left[\frac{\xi-2 K_{2}^{A}}{\alpha_{2}} \mid \Omega_{2}^{A}\right]+E\left[\frac{2 \alpha_{1} K_{2}^{A}-2 \alpha_{2}\left(K_{1}^{A}+K_{f}^{A}\right)-\xi\left(\alpha_{1}-a \alpha_{2}\right)}{\alpha_{2}\left(\alpha_{1}+\alpha_{2}\right)} \mid \Omega_{2}^{A}\right]
\end{aligned}
$$

Recalling that $\Omega_{5}^{A}=\emptyset$ and observing that $\left(\Omega_{2}^{A} \cup \Omega_{3}^{A} \cup \Omega_{4}^{A}\right)=\left\{\xi>2 K_{2}^{A}\right\}$, we can write the KKT condition in Eq. (68) as follows:

$$
\begin{aligned}
& \frac{1}{\alpha_{2}} E\left[\xi-2 K_{2}^{A} \mid \Omega_{2}^{A}\right] \operatorname{Pr}\left(\Omega_{2}^{A}\right)+\frac{1}{\alpha_{2}} E\left[\frac{2 \alpha_{1} K_{2}^{A}-2 \alpha_{2}\left(K_{1}^{A}+K_{f}^{A}\right)-\xi\left(\alpha_{1}-a \alpha_{2}\right)}{\left(\alpha_{1}+\alpha_{2}\right)} \mid \Omega_{2}^{A}\right] \operatorname{Pr}\left(\Omega_{2}^{A}\right) \\
& +\frac{1}{\alpha_{2}} E\left[\xi-2 K_{2}^{A} \mid \Omega_{3}^{A}\right] \operatorname{Pr}\left(\Omega_{3}^{A}\right)+\frac{1}{\alpha_{2}} E\left[\xi-2 K_{2}^{A} \mid \Omega_{4}^{A}\right] \operatorname{Pr}\left(\Omega_{4}^{A}\right)-\frac{1}{\alpha_{2}} 2 K_{f}^{A} \operatorname{Pr}\left(\Omega_{4}^{A}\right)=c_{2} \\
& \Rightarrow E\left[\xi-2 K_{2}^{A} \mid \xi>2 K_{2}^{A}\right] \operatorname{Pr}\left(\xi>2 K_{2}^{A}\right) \\
& =\alpha_{2} c_{2}+2 K_{f}^{A} \operatorname{Pr}\left(\Omega_{4}^{A}\right)-\underbrace{E\left[\frac{2 \alpha_{1} K_{2}^{A}-2 \alpha_{2}\left(K_{1}^{A}+K_{f}^{A}\right)-\xi\left(\alpha_{1}-a \alpha_{2}\right)}{\alpha_{1}+\alpha_{2}} \mid \Omega_{2}^{A}\right]}_{\left.<0 \text { (by definition of } \Omega_{2}^{A}\right)} \operatorname{Pr}\left(\Omega_{2}^{A}\right) \\
& >\alpha_{2} c_{2}
\end{aligned}
$$


Thus, Eq.s (71) and (80) imply that

$$
K_{2}^{A}<K_{2}^{I}
$$

\section{E.2.4 Proof of $K_{2}^{I}<K_{2}^{A}+K_{f}^{A}$}

Observe that:

$$
\begin{aligned}
& E\left[\frac{\xi(1+a)-2 K_{1}^{A}-2 K_{2}^{A}-2 K_{f}^{A}}{\alpha_{1}+\alpha_{2}}\right] \\
& =E\left[\frac{\xi-2\left(K_{2}^{A}+K_{f}^{A}\right)}{\alpha_{2}} \mid \Omega_{2}^{A}\right] \operatorname{Pr}\left(\Omega_{2}^{A}\right) \\
& +E\left[\frac{2 \alpha_{1}\left(K_{2}^{A}+K_{f}^{A}\right)-2 \alpha_{2} K_{1}^{A}-\xi\left(\alpha_{1}-a \alpha_{2}\right)}{\alpha_{2}\left(\alpha_{1}+\alpha_{2}\right)} \mid \Omega_{2}^{A}\right] \operatorname{Pr}\left(\Omega_{2}^{A}\right)
\end{aligned}
$$

Since $K_{2}^{A}>\frac{K_{1}^{A}+K_{f}^{A}}{a}$, it is easy to show that $2 K_{2}^{A}+2 K_{f}^{A}<\frac{2 \alpha_{1} K_{2}^{A}+2 \alpha_{1} K_{f}^{A}-2 \alpha_{2} K_{1}^{A}}{\alpha_{1}-a \alpha_{2}}$.

There are two cases to consider:

Case 1: $2 K_{2}^{A}+2 K_{f}^{A} \leq \frac{2 \alpha_{1} K_{2}^{A}-2 \alpha_{2}\left(K_{1}^{A}+K_{f}^{A}\right)}{\alpha_{1}-a \alpha_{2}}$

In this case, the demand space can be decomposed into Figure 4. KKT condition in Eq. (68) can be written as:

$$
\begin{aligned}
& E\left[\frac{\xi-2\left(K_{2}^{A}+K_{f}^{A}\right)}{\alpha_{2}} \mid \Omega_{2}^{A}\right] \operatorname{Pr}\left(\Omega_{2}^{A}\right)+E\left[\frac{2 \alpha_{1}\left(K_{2}^{A}+K_{f}^{A}\right)-2 \alpha_{2} K_{1}^{A}-\xi\left(\alpha_{1}-a \alpha_{2}\right)}{\alpha_{2}\left(\alpha_{1}+\alpha_{2}\right)} \mid \Omega_{2}^{A}\right] \operatorname{Pr}\left(\Omega_{2}^{A}\right) \\
& +E\left[\frac{\xi-2 K_{2}^{A}}{\alpha_{2}} \mid \Omega_{3}^{A^{\prime}}\right] \operatorname{Pr}\left(\Omega_{3}^{A^{\prime}}\right)+E\left[\frac{\xi-2 K_{2}^{A}-2 K_{f}^{A}}{\alpha_{2}} \mid \Omega_{3}^{A^{\prime \prime}}\right] \operatorname{Pr}\left(\Omega_{3}^{A^{\prime \prime}}\right) \\
& +\frac{2 K_{f}^{A}}{\alpha_{2}} \operatorname{Pr}\left(\Omega_{3}^{A^{\prime \prime}}\right)+E\left[\frac{\xi-2 K_{2}^{A}-2 K_{f}^{A}}{\alpha_{2}} \mid \Omega_{4}^{A}\right] \operatorname{Pr}\left(\Omega_{4}^{A}\right)=c_{2} \\
& \Rightarrow E\left[\xi-2\left(K_{2}^{A}+K_{f}^{A}\right) \mid \xi>2 K_{2}^{A}+2 K_{f}^{A}\right] \operatorname{Pr}\left(\xi>2 K_{2}^{A}+2 K_{f}^{A}\right) \\
& =\alpha_{2} c_{2}-2 K_{f}^{A} \operatorname{Pr}\left(\Omega_{3}^{A^{\prime \prime}}\right)-\underbrace{E\left[\xi-2 K_{2}^{A} \mid \Omega_{3}^{A^{\prime}}\right]}_{>0 \text { (by definition of } \Omega_{3}^{A^{\prime}}} \operatorname{Pr}\left(\Omega_{3}^{A^{\prime}}\right)
\end{aligned}
$$




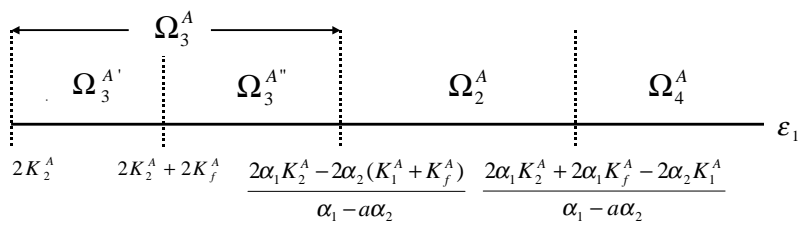

Figure 4: The demand space at solution $\vec{K}^{A}$ for case 1 .

$$
\begin{aligned}
& -\underbrace{E\left[\frac{2 \alpha_{1}\left(K_{2}^{A}+K_{f}^{A}\right)-2 \alpha_{2} K_{1}^{A}-\xi\left(\alpha_{1}-a \alpha_{2}\right)}{\alpha_{1}+\alpha_{2}} \mid \Omega_{2}^{A}\right]}_{\left.>0 \text { (by definition of } \Omega_{2}^{A}\right)} \operatorname{Pr}\left(\Omega_{2}^{A}\right) \\
& <\alpha_{2} c_{2}
\end{aligned}
$$

Thus, Eq.s (71) and (83) imply that:

$$
K_{2}^{A}+K_{f}^{A}>K_{2}^{I}
$$

Case 2: $2 K_{2}^{A}+2 K_{f}^{A}>\frac{2 \alpha_{1} K_{2}^{A}-2 \alpha_{2}\left(K_{1}^{A}+K_{f}^{A}\right)}{\alpha_{1}-a \alpha_{2}}$

In this case, the demand space can be decomposed into Figure 5. KKT condition in 


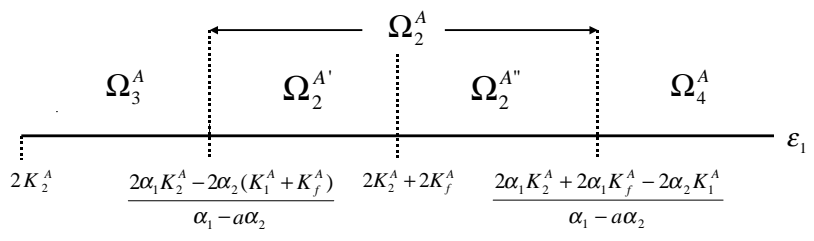

Figure 5: The demand space at solution $\vec{K}^{A}$ for case 2 .

Eq. (68) can be written as:

$$
\begin{aligned}
& \underbrace{E\left[\frac{\xi(1+a)-2\left(K_{1}^{A}+K_{2}^{A}+K_{f}^{A}\right)}{\alpha_{1}+\alpha_{2}} \mid \Omega_{2}^{A^{\prime}}\right]} \operatorname{Pr}\left(\Omega_{2}^{A^{\prime}}\right)+E\left[\frac{\xi-2\left(K_{2}^{A}+K_{f}^{A}\right)}{\alpha_{2}} \mid \Omega_{2}^{A^{\prime \prime}}\right] \operatorname{Pr}\left(\Omega_{2}^{A^{\prime \prime}}\right) \\
& >0 \text { (by definition of } \Omega_{2}^{A^{\prime}} \text { ) } \\
& +\underbrace{E\left[\frac{2 \alpha_{1}\left(K_{2}^{A}+K_{f}^{A}\right)-2 \alpha_{2} K_{1}^{A}-\xi\left(\alpha_{1}-a \alpha_{2}\right)}{\alpha_{2}\left(\alpha_{1}+\alpha_{2}\right)} \mid \Omega_{2}^{A^{\prime \prime}}\right]} \operatorname{Pr}\left(\Omega_{2}^{A^{\prime \prime}}\right) \\
& >0 \text { (by definition of } \Omega_{2}^{A^{\prime \prime}} \text { ) } \\
& +\underbrace{E\left[\frac{\xi-2 K_{2}^{A}}{\alpha_{2}} \mid \Omega_{3}^{A}\right]}_{\left.>0 \text { (by definition of } \Omega_{3}^{A}\right)} \operatorname{Pr}\left(\Omega_{3}^{A}\right)+E\left[\frac{\xi-2\left(K_{2}^{A}+K_{f}^{A}\right)}{\alpha_{2}} \mid \Omega_{4}^{A}\right] \operatorname{Pr}\left(\Omega_{4}^{A}\right)=c_{2} \\
& \Rightarrow E\left[\xi-2\left(K_{2}^{A}+K_{f}^{A}\right) \mid \xi>2 K_{2}^{A}+2 K_{f}^{A}\right] \operatorname{Pr}\left(\xi>2 K_{2}^{A}+2 K_{f}^{A}\right)<\alpha_{2} c_{2}
\end{aligned}
$$


Thus, Eq.s (71) and (85) imply that:

$$
K_{2}^{A}+K_{f}^{A}>K_{2}^{I}
$$

Hence, Eq.s (74), (78), (81) and (86) imply the following relationship:

$$
\frac{K_{1}^{A}}{a}<\frac{K_{1}^{I}}{a}<\frac{K_{1}^{A}+K_{f}^{A}}{a}<K_{2}^{A}<K_{2}^{I}<K_{2}^{A}+K_{f}^{A}
$$

This completes the proof of part 2(b) of Theorem 6.1 .

\section{F Proof of Theorem 6.2}

Letting $\xi_{2}=\xi$ and $\xi_{1}=a-\xi$, our demand space at boundary solution $\vec{K}^{D}$ reduces to:

$$
\begin{aligned}
& \Omega_{1}^{D}=\left\{a-2 K_{1}^{D}<\xi<2 K_{2}^{D}\right\} \\
& \Omega_{2}^{D}=\left\{a>2 K_{1}^{D}+2 K_{2}^{D}, \xi=\frac{\alpha_{2}\left(a-2 K_{1}^{D}\right)+\alpha_{1}\left(2 K_{2}^{D}\right)}{\alpha_{1}+\alpha_{2}}\right\}=\emptyset
\end{aligned}
$$

(since $\xi$ is a continuous random variable)

$$
\begin{aligned}
& \Omega_{3}^{D}=\left\{\xi>2 K_{2}^{D}, \xi\left(\alpha_{1}+\alpha_{2}\right)<2 \alpha_{1} K_{2}^{D}-2 \alpha_{2} K_{1}^{D}+a \alpha_{2}\right\} \\
& \Omega_{4}^{D}=\left\{\xi<a-2 K_{1}^{D}, \xi\left(\alpha_{1}+\alpha_{2}\right)>2 \alpha_{1} K_{2}^{D}-2 \alpha_{2} K_{1}^{D}+a \alpha_{2}\right\} \\
& \Omega_{5}^{D}=\left\{\xi>a-2 K_{1}^{D}, \xi>2 K_{2}^{D}\right\} \\
& \Omega_{6}^{D}=\left\{\xi<a-2 K_{1}^{D}, \xi<2 K_{2}^{D}\right\}
\end{aligned}
$$


Thus, at boundary solution $\vec{K}^{D}$, the first-order KKT necessary \& sufficient conditions, given in Theorem 5.1, reduce to the following:

$$
\begin{aligned}
& \text { KKT - 1 : } \\
& E\left[\frac{a-2 K_{1}^{D}-\xi}{\alpha_{1}} \mid \Omega_{3}^{D}\right] \operatorname{Pr}\left(\Omega_{3}^{D}\right)+E\left[\frac{a-2 K_{1}^{D}-\xi}{\alpha_{1}} \mid \Omega_{4}^{D}\right] \operatorname{Pr}\left(\Omega_{4}^{D}\right) \\
& +E\left[\frac{a-2 K_{1}^{D}-\xi}{\alpha_{1}} \mid \Omega_{6}^{D}\right] \operatorname{Pr}\left(\Omega_{6}^{D}\right)=c_{1}
\end{aligned}
$$

KKT - 2 :

$E\left[\frac{\xi-2 K_{2}^{D}}{\alpha_{2}} \mid \Omega_{3}^{D}\right] \operatorname{Pr}\left(\Omega_{3}^{D}\right)+E\left[\frac{\xi-2 K_{2}^{D}}{\alpha_{2}} \mid \Omega_{4}^{D}\right] \operatorname{Pr}\left(\Omega_{4}^{D}\right)+E\left[\frac{\xi-2 K_{2}^{D}}{\alpha_{2}} \mid \Omega_{5}^{D}\right] \operatorname{Pr}\left(\Omega_{5}^{D}\right)=c_{2}(88$

KKT - 3 :

$$
\begin{aligned}
& E\left[\frac{a-2 K_{1}^{D}-\xi}{\alpha_{1}} \mid \Omega_{3}^{D}\right] \operatorname{Pr}\left(\Omega_{3}^{D}\right)+E\left[\frac{\xi-2 K_{2}^{D}}{\alpha_{2}} \mid \Omega_{4}^{D}\right] \operatorname{Pr}\left(\Omega_{4}^{D}\right) \\
& +E\left[\frac{\xi-2 K_{2}^{D}}{\alpha_{2}} \mid \Omega_{5}^{D}\right] \operatorname{Pr}\left(\Omega_{5}^{D}\right)+E\left[\frac{a-2 K_{1}^{D}-\xi}{\alpha_{1}} \mid \Omega_{6}^{D}\right] \operatorname{Pr}\left(\Omega_{6}^{D}\right)=c_{f}-v_{f}=\underline{c}_{f}
\end{aligned}
$$

Consider the optimal solution, $K_{i}^{I}$, to the independent problem for demand-class $i, i=$ 1,2 (Problem $P_{2}(i)$ ). By definition, solution $\left(K_{1}^{I}, K_{2}^{I}\right)$ satisfies conditions KKT-1 and KKT-2. If this solution also satisfies condition KKT-3, then by Theorem 5.1, it must be the optimal solution, with the optimal investment vector being $\vec{K}^{D}=\left(K_{1}^{I}, K_{2}^{I}, 0\right)$. Otherwise, the optimal solution must have $K_{f}^{*}>0$. Thus, in the following, letting $\left(K_{1}^{D}, K_{2}^{D}\right)=$ $\left(K_{1}^{I}, K_{2}^{I}\right)$, we analyze whether or not this solution satisfies KKT-3. There are three possible cases:

1. If $2 K_{1}^{I}+2 K_{2}^{I}=a$, then $\Omega_{1}^{D}=\Omega_{2}^{D}=\Omega_{3}^{D}=\Omega_{4}^{D}=\emptyset$. Then, KKT conditions in Eq.s (87)-(89) imply that $\underline{c}_{f}=c_{1}+c_{2}$. Since $c_{f}<c_{1}+c_{2}$ by definition, it follows by Lemma 5.2 that $K_{f}^{*}>0$ in the optimal solution. 
2. If $2 K_{1}^{I}+2 K_{2}^{I}>a$, then $\Omega_{2}^{D}=\Omega_{3}^{D}=\Omega_{4}^{D}=\emptyset$. Then, KKT conditions in Eq.s (87)-(89) imply that $\underline{c}_{f}=c_{1}+c_{2}$, and therefore, it follows by Lemma 5.2 that $K_{f}^{*}>0$ in the optimal solution.

3. If $2 K_{1}^{I}+2 K_{2}^{I}<a$, then $\Omega_{1}^{D}=\Omega_{2}^{D}=\emptyset$. Then, KKT conditions in Eq.s (87)-(89) imply that $\underline{c}_{f}=c_{1}+c_{2}-\left\{\frac{1}{\alpha_{1}} E\left[a-2 K_{1}^{I}-\xi \mid \Omega_{4}^{D}\right] \operatorname{Pr}\left(\Omega_{4}^{D}\right)+\frac{1}{\alpha_{2}} E\left[\xi-2 K_{2}^{I} \mid \Omega_{3}^{D}\right] \operatorname{Pr}\left(\Omega_{3}^{D}\right)\right\}$. By Lemma $5.2, K_{f}^{*}>0$ in the optimal solution only if $c_{f}<\underline{c}_{f}$. Also, by our assumption, $c_{1}, c_{2}<c_{f}<c_{1}+c_{2}$. Thus, in order to show that $K_{f}^{*}$ can be positive in the optimal solution, we need to show that:

$$
\frac{1}{\alpha_{1}} E\left[a-2 K_{1}^{I}-\xi \mid \Omega_{4}^{D}\right] \operatorname{Pr}\left(\Omega_{4}^{D}\right)+\frac{1}{\alpha_{2}} E\left[\xi-2 K_{2}^{I} \mid \Omega_{3}^{D}\right] \operatorname{Pr}\left(\Omega_{3}^{D}\right)<\min \left(c_{1}, c_{2}\right) .
$$

(i) First, we show that

$$
\frac{1}{\alpha_{1}} E\left[a-2 K_{1}^{I}-\xi \mid \Omega_{4}^{D}\right] \operatorname{Pr}\left(\Omega_{4}^{D}\right)+\frac{1}{\alpha_{2}} E\left[\xi-2 K_{2}^{I} \mid \Omega_{3}^{D}\right] \operatorname{Pr}\left(\Omega_{3}^{D}\right)<c_{1}
$$

Observe that by Eq. (87), this is equivalent to showing that:

$$
\underbrace{E\left[\xi\left(\alpha_{1}+\alpha_{2}\right)-2 \alpha_{1} K_{2}^{I}+2 \alpha_{2} K_{1}^{I}-a \alpha_{2} \mid \Omega_{3}^{D}\right]}_{\left.<0 \text { (by definition of } \Omega_{3}^{D}\right)} \operatorname{Pr}\left(\Omega_{3}^{D}\right)<\alpha_{2} \underbrace{E\left[a-2 K_{1}^{I}-\xi \mid \Omega_{6}^{D}\right]}_{\left.>0 \text { (by definition of } \Omega_{6}^{D}\right)} \operatorname{Pr}\left(\Omega_{6}^{D}\right),
$$

which always holds by definitions of $\Omega_{3}^{D}$ and $\Omega_{6}^{D}$. (ii) Next, we show that

$$
\frac{1}{\alpha_{1}} E\left[a-2 K_{1}^{I}-\xi \mid \Omega_{4}^{D}\right] \operatorname{Pr}\left(\Omega_{4}^{D}\right)+\frac{1}{\alpha_{2}} E\left[\xi-2 K_{2}^{I} \mid \Omega_{3}^{D}\right] \operatorname{Pr}\left(\Omega_{3}^{D}\right)<c_{2}
$$


Observe that by Eq. (88), this is equivalent to showing that:

$$
\underbrace{E\left[\alpha_{2}\left(a-2 K_{1}^{I}\right)-\xi\left(\alpha_{1}+\alpha_{2}\right)+2 \alpha_{1} K_{2}^{I} \mid \Omega_{4}^{D}\right]}_{\left.<0 \text { (by definition of } \Omega_{4}^{D}\right)} \operatorname{Pr}\left(\Omega_{4}^{D}\right)<\alpha_{1} \underbrace{E\left[\xi-2 K_{2}^{I} \mid \Omega_{5}^{D}\right]}_{>0\left(\text { by definition of } \Omega_{5}^{D}\right.} \operatorname{Pr}\left(\Omega_{5}^{D}\right),
$$

which always holds by definitions of $\Omega_{4}^{D}$ and $\Omega_{5}^{D}$. Thus, $K_{f}^{*}$ may be positive in the optimal solution.

Finally, to prove the equivalence relations, we substitute $\xi_{2}=\xi$ and $\xi_{1}=a-\xi$ in KKT-1 and KKT-2 and obtain:

$$
\begin{array}{ll}
\mathbf{K K T}-\mathbf{1}: & E\left[a-2 K_{1}^{I}-\xi \mid \xi<a-2 K_{1}^{I}\right] \operatorname{Pr}\left(\xi<a-2 K_{1}^{I}\right)=\alpha_{1} c_{1} \\
\mathbf{K K T}-\mathbf{2}: & E\left[\xi-2 K_{2}^{I} \mid \xi>2 K_{2}^{I}\right] \operatorname{Pr}\left(\xi>2 K_{2}^{I}\right)=\alpha_{2} c_{2}
\end{array}
$$

Consider again the condition that $2 K_{1}^{I}+2 K_{2}^{I} \geq a$, or equivalently, $2 K_{2}^{I} \geq a-2 K_{1}^{I}$. Thus, we can write:

$$
\begin{aligned}
E\left[\xi-2 K_{2}^{I} \mid \xi>2 K_{2}^{I}\right] \operatorname{Pr}\left(\xi>2 K_{2}^{I}\right) \leq & E\left[\xi-\left(a-2 K_{1}^{I}\right) \mid \xi>a-2 K_{1}^{I}\right] \operatorname{Pr}\left(\xi>a-2 K_{1}^{I}\right) \\
& +E\left[\xi-\left(a-2 K_{1}^{I}\right) \mid \xi<a-2 K_{1}^{I}\right] \operatorname{Pr}\left(\xi<a-2 K_{1}^{I}\right) \\
& +E\left[a-2 K_{1}^{I}-\xi \mid \xi<a-2 K_{1}^{I}\right] \operatorname{Pr}\left(\xi<a-2 K_{1}^{I}\right) \\
\Rightarrow \alpha_{2} c_{2} \leq & \alpha_{1} c_{1}+E\left[\xi-\left(a-2 K_{1}^{I}\right)\right], \quad \text { by Eq.s (90) and (91) } \\
\Rightarrow \alpha_{2} c_{2}-\alpha_{1} c_{1} \leq & E[\xi]-\left(a-2 K_{1}^{I}\right)
\end{aligned}
$$

Letting $\Delta^{t h} \equiv E[\xi]-\left(a-2 K_{1}^{I}\right)$ leads to the relation. The other case is obtained similarly. This completes the proof. 


\section{Vita}

Qiong Wang was born on January 27, 1969 in Xi'an, China. She obtained her Bachelor's degree in Economic Geography from North Western University, China in 1991. She continued her graduate study in the School of Management at Xi'an Jiaotong University for three years. After completing her MS in Management Systems Engineering, she worked in the Research Center of Systems Analysis (RCSA), Research Institute of Manufacturing Science \& Technology, Ministry of Manufacturing Industry, Beijing, China. As a researcher and a consultant, she led or took part in more than ten research projects focusing on development strategies of advanced manufacturing technologies, supply chain management, logistics, etc. Because of her excellent performance, she received the National Award of Science \& Technology Development (China) in 1999 and had been promoted to a team leader in charge of the Development Strategy Division in RCSA from July 1997 to May 2000.

In August 2000, she started to pursue her Master's degree in Industrial Engineering at Virginia Tech. She worked as a Research Assistant with her advisor Dr. Bish on the Flexible Capacity Management Project (sponsored by NSF, co-operating with General 
Motors, R\&D Division), and a Teaching Assistant in the Grado Department of Industrial and Systems Engineering for two semesters. She did a presentation at 2002 Manufacturing and Service Operations Management Conference, and submitted one paper to Operations Research.

She will graduate in December 2002. Her thesis is "Optimal Investment Strategies for Flexible Resources, Considering Pricing and Correlated Demands." Her research interests are in Operations Research, Logistics, and Supply Chain Management. 